\title{
ABSTRACTS FROM THE 2011 EVDI ANNUAL MEETING
}

\author{
London, England \\ August 30-September 3, 2011
}

Veterinary Radiology \& Ultrasound, Vol. 52, No. 6, 2011, pp 674-705.

\section{COMPUTED TOMOGRAPHIC EVALUATION OF THE CANINE SALIVARY GLAND APPARATUS}

T. Liuti, A.I. de Castro Marques, T. Schwarz. Hospital for Small Animals, The Royal (Dick) School of Veterinary Studies, University of Edinburgh, UK

\section{Introduction/Purposes:}

Salivary gland pathology is rarely reported in the veterinary literature. Contrast radiography has been traditionally used to characterize the salivary gland apparatus and aid diagnosis. Computed tomography (CT) eliminates organ superimposition maximizing visibility of soft tissue and bone structures. The aim of this study was to characterize the anatomical appearance of nondiseased canine salivary gland apparatus using contrast-enhanced CT sialography.

Materials and Methods:

Five dogs, euthanized on humane grounds, for reasons unrelated to diseases of the head, were used for anatomical and CT evaluation. In the first part of the study, ethylene blue was injected in the mandibular, sublingual, parotid, and zygomatic salivary ducts of two dogs. Surgical dissection was performed and the anatomical location, appearance, and orientation of the salivary gland apparatus were photographed. In the second part of the study, 2 $\mathrm{mm}$ thick, sequential CT images of three dogs were acquired before and after injection of iodine-based, ionic, contrast medium (iothalamate meglumine, Conray $\left.250^{(}\right)$. Bone and soft-tissue reconstructions were available for evaluation. Duct cannulation was achieved by a staff surgeon using a 26-g, 19-mm intravenous catheter (Anicath IV Cannula ${ }^{\circledR}$ ): the salivary papilla was first identified and the catheter sleeve was slowly advanced. Five to ten millilitres of contrast agent was injected into each cannulated duct.

millilitres

Mandibular, sublingual, parotid, and zygomatic glands and respective ducts were easily recognized and followed on $\mathrm{CT}$ images after injection of contrast medium. Orientation, size, and anatomic appearance of the salivary apparatus on CT images were consistent with the anatomical dissection findings. The salivary glands were all identified as lobulated and highly contrast enhancing.

Discussion/Conclusion:

The results of this study show that contrast CT is an effective method of outlining nondiseased salivary gland apparatus. The salivary duct was easily identified in all cases and these images may facilitate future diagnosis of salivary gland pathology. A prospective clinical study is currently being undertaken at the same institution.

\section{CT SIALOGRAPHY IN THE DOG-A CADAVER STUDY}

S. Weidner, N. Pfammatter, A. Probst, S. Kneissl. Department of Small Animals and Horses, Division of Diagnostic Imaging and The Department of Pathobiology, Anatomy and Histology, University of Veterinary Medicine, Vienna, Austria

\section{Introduction/Purpose:}

To document computed tomography (CT) topography of salivary glands and their ducts in dogs, a retrograde filling with methylcellulose and iodinated contrast medium was performed in three cadavers.

\section{Methods:}

After plain multislice CT of three canine cadaver heads, the orifices of the salivary ducts were cannulated with a lacrimal cannula. The device was fixed with submucosal sutures, and an extension set was mounted on it. A mixture of methylcellulose and iodinated contrast medium was injected with force. Subsequently, contrast CT was performed. Technical settings were $130 \mathrm{kV}$ and $96 \mathrm{~mA}$, and the estimated slice thickness was $0.8 \mathrm{~mm}$. Data were archived in a picture archiving and communication system. Multiplanar reconstructions and surface models were calculated using a syngo MultiModality Workplace.

models wer:

Demarcation of the parotid, mandibular, and zygomatic glands was achieved. Surrounding structures were imaged without beam hardening artifacts. Landmarks for the parotid, mandibular, and zygomatic glands were the external acoustic canal, the mandibular angle and the pterygopalatine fossa, respectively. Sialograms of the parotid, mandibular, and zygomatic ducts were achieved, whereas contrast enhancement of the sublingual glands and their ducts was not possible.
Discussion:

To our knowledge, only two studies have been published on the normal radiographic appearance of salivary glands and ducts in the $\operatorname{dog}^{1,2}$. CT sialography has not been documented in veterinary medicine. Diameter of the lacrimal cannula $(\sim 1.4 \mathrm{~mm})$ may have been too large to cannulate the major sublingual duct. Alternatively, the mixture of methylcellulose (MC) and water may have been too viscous to enter smaller ducts. In humans, bitter substances increase salivation and ease retrograde cannulation. This technique could be of help in living dogs as well. Compared with other invasive techniques, sialography is relatively simple and could provide valuable information, especially when imaged with CT. Additionally, it would preserve salivary duct integrity and continuation when sialoliths or salivary duct ruptures and other problems are suspected.

References:

1. Harvey CE, O'Brien JA, Rossman LE. Oral, dental, pharyngeal and salivary gland disorders. In: Ettinger S. J. (ed.) Textbook of Veterinary Internal Medicine, Vol. II. Diseases of the Dog and Cat, 2nd edn. Philadelphia: Saunders. 1983; pp. 1177-1187.

2. Tadjalli M, Dehghani SN, Basiri M. Sialography in dog: normal appearance. Vet Arh 2004;74:225-233.

\section{EFFECT OF SKULL CONFORMATION ON PARAMETERS OF THE BONY NASOPHARYNX IN DOGS}

A.K. Hussein, J. Penderis, M. Sullivan. School of Veterinary Medicine, University of Glasgow,

\section{Introduction:}

Apnoea, hypopnoea, oxygen desaturation, otitis media, snoring, and obstruction sleep apnoea syndrome (OSAS) have been associated with narrowing of the bony nasopharynx, oropharynx, and soft palate in men and animals. A protrusion of the nasoturbinates into the pharynx may also lead to obstruction in the caudal choanal area. This study aimed to determine if there was a correlation between the shape of the bony nasopharynx (at a specific level) and different skull conformations.

Methods:

Thirty-two dogs (12 Cavalier King Charles spaniels, 3 boxers, 3 shih-tzus, 2 chihuahuas, 2 Labradors, 2 West Highland white terriers, and 1 deerhound, English springer spaniel, giant schnauzer, mastiff, miniature schnauzer, pointer, pug, Staffordshire bull terrier) with no clinical signs or pathology of either cranial or nasopharyngeal cavities were recruited to this study. Using a 1.5-T magnet, the following measurements were made on T1w/T2w images: (1) Evans and Stockard skull indices; (2) area and shape of the bony nasopharynx (transverse plane) at the level of caudal nasal spine of the hard palate; (3) height of the nasopharynx (mid-sagittal plane) calculated by drawing a line through the midline point of the olfactory bulb area to intersect with a base line at a right angle (a line passing through the intercondylar notch caudally and hard palate rostrally); (4) olfactory bulb angulation and orientation (midline sagittal plane) in relation to the base.

Results:

The findings revealed (1) a significant relationship between the area of the nasopharynx in the transverse plane and both body weight and skull phenotype [Evans' index ( $P$ $0.0001)$, Stockard's index $(P<0.0001)$, and olfactory bulb angulation $(P<0.0001)$ ], (2) the height of the nasopharynx correlated with the skull phenotype $(P<0.0001)$ but not the body weight $(P=0.1355)$ measured in the mid-sagittal plane. Three main shapes were identified at transverse section: apart from oval shape, peanut shell, and mouth/inverted mouth-like shapes. When the area, height, and shape were compared to the orientation of the olfactory bulb, a more ventral olfactory bulb orientation was associated with (1) smaller area, (2) a peanut-shell-like shape (which sometimes included nasoturbinates) to oval in a more rostrally orientated olfactory bulb. The area (corrected for body weight) was $4.1 \pm 0.6$, $3.7 \pm 0.4,3.1 \pm 0.5$ for oval, mouth/inverted mouth and peanut shell shapes, respectively. It was noticed that the irregular shape of the bony part of the nasopharynx on transverse section largely occurred in dogs with a high cephalic index (i.e., more brachycephalic) due to a bent hard palate and nasopharyngeal roof (which is formed by either the vomer or presphenoid bones associating with the caudal point of nasal spine of hard palate ventrally), sometimes with nasoturbinates present in the cavity.

Conclusion:

More brachycephalic breeds have not only a smaller nasopharyngeal area, but also a different shape to other skull types. Selective breeding may contribute to normalizing (or exacerbating) these features and result in a reduction in respiratory disorders. 


\section{THE COMPUTED TOMOGRAPHIC APPEARANCE OF NORMAL CANINE AND FELINE NASAL TURBINATES}

R. Uosyte, D.A. Gunn-Moore, E. Fraga Manteiga, D. W. Shaw, T. Schwarz. The Royal (Dick) School of Veterinary Studies, Division of Clinical Veterinary Sciences, Roslin, UK

Introduction:

Visibility of nasal turbinates in computed tomography (CT) is used for the diagnosis of canine and feline nasal disease. The purpose of this study was to determine the influence of technical and patient factors on normal turbinate visibility.

Methods:

Transverse rostral and caudal nasal slabs of a feline and canine cadaver were bandsawed and CT-scanned before and after submersion in water with the following variables: slice width, pitch, sequential/helical mode, and image reconstruction algorithm. Turbinate visibility was assessed objectively with manual tracing. Object contrast was determined via standard deviation from mean attenuating values. Different window settings were tested.

Results:

Turbinate visibility is higher in the dog versus cat, caudally versus rostrally, native versus water-submersed, correlating with object contrast magnitude. Turbinates appear more abundant and better delineated in thin-slice, high-frequency image-reconstruction algorithm, sequential mode, low-pitch images with wide and low window settings.

Discussion:

Optimal CT settings are essential for accurate turbinate assessment. Nasal fluid masks turbinate detail mimicking turbinate destruction. This is particularly critical for differentiation of feline rhinitis from aggressive neoplasia and mycosis.

\section{CT FINDINGS OF NASAL ADENOCARCINOMA IN FOUR MOON BEARS (URSUS THIBETANUS)}

H. Bacon, N. Webster, M. Bando. Animals Asia Foundation, Chengdu, China and Veterinary Imaging Associates, Sydney, Australia

Introduction:

The purpose of this study was to describe the CT findings of confirmed nasal adenocarcinoma in four moon bears (Ursus thibetanus)

Methods:

Four bears with clinical signs of nasal disease were evaluated at the Animals Asia Foundation between October 2008 and January 2010. The bears were all adults; however, specific aging was not possible due to unknown birth history. Clinical signs were subtle in the early stages of disease and included reduced activity, decreased appetite, and bilateral mucopurulent of disease and included reduced activity, decreased appetite, and bilateral mucopurulent
nasal discharge. Clinical signs progressed to mucosanguinous discharge and head pressing or placing a paw over the rostral cranium (head holding). The bears were anesthetized for examination and CT study of the head. One bear had a thoracic CT performed. Nasa biopsies were taken using biopsy forceps and a blind biopsy technique. The tissue was sent for histological evaluation. One bear with no clinical signs of nasal disease had a CT of the head performed as part of the clinical work up. This was used as a control study.

Results:

CT Findings: All four bears had an extensive, aggressive soft-tissue mass within the nasa chambers. The histological findings were consistent with adenocarcinoma. The bear with no clinical signs of nasal disease had a normal nasal CT.

\begin{tabular}{llllll}
\hline & $\begin{array}{l}\text { Cribiform } \\
\text { plate } \\
\text { destruction }\end{array}$ & $\begin{array}{l}\text { Extension into } \\
\text { calvarium and } \\
\text { mass effect }\end{array}$ & $\begin{array}{l}\text { Osteolysis } \\
\text { (maxilla, nasal } \\
\text { septum) }\end{array}$ & $\begin{array}{l}\text { Extension } \\
\text { into frontal } \\
\text { sinus }\end{array}$ & $\begin{array}{l}\text { Extension } \\
\text { into } \\
\text { Nasopharynx }\end{array}$ \\
\hline Bear 1 & $\mathrm{Y}$ & $\mathrm{N}$ & $\mathrm{Y}$ & $\mathrm{Y}$ & $\mathrm{Y}$ \\
Bear 2 & $\mathrm{Y}$ & $\mathrm{Y}$ (Mild) & $\mathrm{Y}$ & $\mathrm{Y}$ & $\mathrm{Y}$ \\
Bear 3 & $\mathrm{Y}$ & $\mathrm{Y}$ & $\mathrm{Y}$ & $\mathrm{Y}$ & $\mathrm{Y}$ \\
Bear 4 & $\mathrm{Y}$ & $\mathrm{Y}$ & $\mathrm{Y}$ & $\mathrm{Y}$ & $\mathrm{Y}$ \\
\hline
\end{tabular}

\section{Discussion:}

The CT findings of nasal adenocarcinoma in four moon bears consists of nasal mass effect turbinate destruction, osteolysis, and extension into adjacent cavities. The CT findings are more advanced than usually seen in companion animals due to the difficulty in detecting subtle clinical signs in a nondomesticated and very stoic species. We would recommend that any collections holding captive bears pay close attention to nasal discharge as a sign of potential nasal carcinoma, even in bears exhibiting normal behaviors.

\section{OPTIMIZED CANINE DENTAL COMPUTED TOMOGRAPHIC PROTOCOL}

M.C. Esmans ${ }^{1}$, J.W. Soukup ${ }^{2}$, T. Schwarz ${ }^{1}{ }^{1}$ The Royal (Dick) School of Veterinary Studies, Roslin, UK, ${ }^{2}$ The University of Wisconsin-Madison, MA

\section{Introduction:}

Computed tomography (CT) is a frequently used diagnostic tool for the oral cavity in dogs, including the dental apparatus. Very high anatomic detail is necessary for dental diagnostics. Optimized CT settings to achieve this have not been published. The purpose of this study was to establish an optimized imaging protocol for canine dental imaging using single- and multidetector-row CT.

Methods:

CT was performed on two canine cadaver heads with a one- and four-slice CT unit using a different slice thickness, table increment, pitch, image reconstruction algorithm, sequential, and helical scan modes. Images were blindly reviewed by two examiners.

Results:

A sequential mode, 1-mm slice thickness and interval with high-frequency image reconstruction algorithm were optimal. A helical mode 1 -mm slice thickness also revealed acceptable image quality. In thin-slice helical mode, no influence of pitch (max pitch of 2) was identified. Both CT scanners showed equal results.

\section{Discussion:}

Thin-slice sequential mode CT images reveal optimal dental anatomic detail. Helical scanning can be performed with moderate loss of anatomic detail.

\section{MAGNETIC RESONANCE IMAGING AND ULTRASOUND FOR DETECTION OF NORMAL PARATHYROID GLANDS AND HYPERFUNCTIONING PARATHYROID NODULES IN DOGS}

R. Cruz, S. Kruth, T. Gibson, S. Nykamp. University of Guelph, Canada

Introduction/Purpose:

Primary hyperparathyroidism (PHPTH) is uncommon in dogs. Ultrasound is commonly used to identify parathyroid nodules, however, some researchers have reported that hyperfunctional parathyroid nodules (hfPTHN) are not always identified and in some cases they cannot be differentiated from thyroid nodules due the close anatomic relationship. In humans, MRI has been reported to have sensitivity and specificity similar or superior compared to $99 \mathrm{mTc}$ methoxyisonitrile (MIBI) scintigraphy and US. The use of MRI to evaluate parathyroid glands (PTHg) has not been previously studied in dogs. Objectives: (1) to describe normal MR anatomy of the PTHg in dogs; (2) to compare MRI and US for the detection of hfPTHN in dogs with PHPTH.

Methods:

The PTHgs were imaged with MRI and US in six control dogs (two small, two medium and two large dogs) and three hyperparathyroid (HPTH) dogs. US was performed with an 8-12 MHz linear transducer. T1, T2, and diffusion-weighted MR images were acquired with a 1.5-T unit. PTHg was considered to be a nodule if it was greater than $2 \mathrm{~mm}$. hfPTHNs were surgically resected and submitted for histopathology. Images were reviewed by two blinded board-certified radiologists. Presence of the parathyroid, size, echogenicity, or signal intensity, and a subjective assessment of normal or abnormal were recorded.

Results:

Control dogs were normal on physical examination and had serum calcium, i-calcium, PTH rPTH levels within reference values. According to one of the observers, none of the PTHgs was observed on any of the MRI sequences in normal dogs. According to the other observer, the two cranial PTHgs were identified in one large breed dog and the left cranial PTHg was observed in the other large breed dog. In the clinical cases, the radiologists had complete agreement, except for the DWI. An abnormal right cranial PTH nodule in one HPTH dog and a left caudal PTH nodule in another dog were detected. The nodule in the first dog and a left caudal PTH nodule in another dog were detected. The nodule in the first dog
was extrathyroidal (ETh) on US, MRI, and surgery whereas in the second HPTH dog, it was intrathyroidal (ITh) on US and surgery, and ETh on MRI. Adenoma and hyperplasia progressing to adenoma was diagnosed at histopathology, respectively. In the third HPTH dog, two PTH nodules were identified on US, one ITh (left caudal) and the other ETh (right caudal). Only the ETh nodule was present on MRI. This nodule was found on surgery (ETh) whereas the second nodule seen on US could not be found. An adenoma was diagnosed on histopathology. Relative to the thyroid gland, all the nodules were hypoechoic on US, isointense to the thyroid lobes on T1WI, and slightly hyperintense on T2WI with mild contras enhancement compared to thyroid glands. hfPTHN were hypointense compared to thyroid gland on DWI for one reviewer and isointense for the other one. Calcium levels return to normal limits and dogs were free of clinical signs following surgery.

Conclusions:

MRI can detect hfPTHN in dogs. The nodule seen on US but not in MRI can be interpreted as likely to be a thyroid colloid nodule and not an hfPTHN. MRI may be able to discriminate between thyroid colloid nodules and hfPTHN but further evaluation is needed. 


\section{DIFFUSION-WEIGHTED MAGNETIC RESONANCE IMAGING OF SPINAL CORD SPECIMENS FROM DOGS WITH DEGENERATIVE MYELOPATHY COMPARED TO HISTOPATHOLOGY}

M.J. Schmidt, U. Pilatus, A. Oevermann, K.S. Grohmann, M. Vandevelde, M. Kramer. Klinik für Kleintiere-Chirurgie, Justus-Liebig-Universität, Gießen, Germany

\section{Purpose:}

Examination of formalin-fixed spinal cord specimen of dogs with degenerative myelopathy (DM) with diffusion-weighted magnetic resonance imaging (DWI) compared to histopathologic examination to evaluate the benefit of the technique for in vivo studies.

\section{Methods:}

The spinal cord of eight dogs with suspected DM and two unaffected dogs were examined after exenteration and formalin fixation. Formalin does not alter diffusion characteristics of the spinal cord. Thirty $10-\mathrm{cm}$ cuts were examined using a 3-T imaging unit and a wrist coil. T2- and diffusion-weighted images and calculated apparent diffusion coefficient (ADC) mappings were obtained. Three different $b$ values of 500,1000 , and 1500 were used to determine differences in diffusion along each of the three axes.

\section{Results:}

Ten specimens of the suspected DM-affected dogs showed a diffuse increase in signal intensity in DWI and inversion of the signal in ADC. In 10 of the DM specimen, no signal changes could be seen. In unaffected dogs, four specimens showed increased signal intensity, six specimens showed no signal changes. Histopathology revealed classic characteristics of DM in all suspected dogs. Widespread axonal loss, swollen axons with axonal spheroids, and invasion of macrophages into disintegrated myelin sheaths were evident. Nerve fibers were widely replaced by astroglial cells and fibers. The only difference between specimens was the degree of astrogliosis. The unaffected dogs were diagnosed having widespread autolytic areas.

Discussion:

A hyperintense signal in DWI is indicative for decreased diffusion. The reduction in free motion of water can be caused by intramyelinic edema and severe astrogliosis. The difference between the DM-positive specimen that showed hyperintense signals in DWI and those with absent hyperintensities was related to the grade of the astrogliosis. We suspect that the amount of glial cells and fibers have a major influence on the diffusion of water molecules in dogs with DM in our study. These might be different from nonchronic lesions in which axonal loss is the major finding.

Hyperintense signals in the dogs without DM can be explained by autolysis, mechanica trauma, and malacia of the myelon, which is reported to go along with bright signal intensity in DWI. DWI seems to be a beneficial technique for the in vivo diagnosis of DM.

\section{LINEAR, AREA, AND VOLUMETRIC EVALUATION OF DOG LATERAL VENTRICLES USING LOW-FIELD MAGNETIC RESONANCE IMAGING}

S. Borgonovo, D.D. Zani, O. Travetti, M. Moioli, F. Manzoni, D. De Zani, A. Zecconi, M. Di Giancamillo. Faculty of Veterinary Medicine, Milano, Italy

\section{Introduction:}

The aim of the study was to compare both linear and area measurements of lateral ventricles with corresponding volumetric values, in order to evaluate possible differences in the results obtained, according to the age of patients, skull conformations, and presence/absence of any abnormalities causing mass effect on the ventricular system.

\section{Materials and Methods:}

Brains of 135 dogs, aged from 4 months to 15 years, were examined using transverse T1-weighted turbo spin-echo sequences, acquired by a low-field magnet $(0.18 \mathrm{~T}$, Vet-MR Esaote S.p.A., Genova, Italy). Dogs were divided into different groups according to skull conformation (brachycephalic, mesocephalic, and dolicocephalic), age (young and adult groups, under and over 15th month, respectively), and presence/absence of any intracranial lesion (mass and no mass groups) causing mass effect on the ventricular system. Ventricula and brain height $(\mathrm{Vh}, \mathrm{Bh})$ and ventricular and hemispheric area (VA, HA) were measured at interthalamic adhesion level. Total lateral ventricle volume (VVtot) and singular latera ventricle volume (VV) were obtained by thresholding-based segmentation algorithm. The ratio $\mathrm{Vh} / \mathrm{Bh}, \mathrm{VA} / \mathrm{HA}, \mathrm{VV} / \mathrm{Bh}$, and largest/smallest ventricle, in terms of area (rVA) and height $(\mathrm{rVh})$, were calculated. VV/Vh, VA/VV, rVh/rVA, Vh/Bh, and VV/Bh ratio (with previous age differentiation) relationship were statistically investigated by linear regression. For VVtot, differentiation) relationship were statistically investigated by linear regression. For $V$ tot,
$\mathrm{Vh} / \mathrm{Bh}$, and VA/HA ratio-significant differences between age groups, skull conformation, and presence/absence of mass were assessed by GLM procedure.
Results:

Thirty-nine dogs were brachycephalic, 73 mesocephalic, and 23 dolicocephalic. Twenty were classified as young and 115 as adult; 21 dogs were included in the mass group. Asymmetry between ventricles (rVA $>1.5$ ) was found in $24.4 \%$ of investigated cases. Brachycephalic dogs with normal-sized ventricles (Vh/Bh: $0-14 \%$ ) were $41 \%$ and $38.5 \%$ for right and left ventricles, respectively, compared to about $70 \%$ in dogs showing different skul conformations. Significant difference between VVtot brachycephalic and mesocephalic dogs and in $\mathrm{Vh} / \mathrm{Bh}$ ratio between brachycephalic and other skull conformations were observed $(P<0.05)$. In patients with normal-sized ventricles, significant difference was found for $\mathrm{Vh} / \mathrm{Bh}$ and VA/HA ratio between groups mass and no mass $(P<0.05)$. Regression procedure demonstrated a statistical relationship for each compared couple of parameters.

\section{Discussion:}

Prevalence of ventricular asymmetry was lower than previously reported, probably due to sample heterogeneity. More than $50 \%$ of dogs showing ventriculomegaly were brachycephalic and the significant difference observed in $\mathrm{Vh} / \mathrm{Bh}$ ratio between brachycephalic and the other skull conformations, disappeared after sample stratification according to ventricular size classification. Brachycephalic breeds have larger lateral ventricles than other dogs, hence the current lateral ventricle dimensional classification is not appropriate due to ex treme morphologic variability of canine skull and brain. To our knowledge, VV/Bh ratio for determination of lateral ventricle size was not previously reported and we believe that this method could represent a good compromise between reproducibility and repeatability, because brain height, as a linear measurement, showed higher reproducibility and repeatability than manual tracing method as reported in previous studies.

\section{MRI FINDINGS IN 15 ACROMEGALIC CATS}

B. Posch, J. Dobson, M.E. Herrtage. Department of Veterinary Medicine, University of Cambridge, Cambridge, UK

Introduction/Purpose:

Feline acromegaly is characterized by chronic excessive growth hormone secretion, causing insulin-resistant diabetes mellitus, and overgrowth of connective tissue, bone, and viscera. The most common cause is a functional pituitary adenoma. A diagnosis of acromegaly is currently based upon a combination of clinical signs, measurement of growth hormone, and/or insulin-like growth factor-1 (IGF-1) and intracranial imaging. The purposes of this study were to evaluate if pituitary abnormalities were present on MRI in acromegalic cats, to describe the MRI findings, and to assess if specific morphologic criteria can be established. describe the MRI findings,
Materials and Methods:

Medical records between January 2003 and August 2010 were searched for cats in which clinical signs and laboratory findings were compatible with acromegaly, and in which an MR was performed. Inclusion criteria were as follows: elevated serum IGF-1 (>1000 ng/ml) as a surrogate marker of hypersomatotropism and insulin resistant diabetes mellitus. Fifteen cats met the inclusion criteria. The mean age was 10.5 years (range $6-14$ years). Neutered male cats $(14 / 15)$ and domestic short haired (13/15) were overrepresented. MRI of the brain was performed using a $0.2-\mathrm{T}$ scanner. Precontrast and postcontrast T1W and T2W images were acquired in transverse and sagittal planes. MRI findings were reviewed retrospectively. The pituitary gland was measured on postcontrast T1W images using a standardized protocol. The following MRI criteria were used to assess the pituitary gland: size, shape, margin, signal intensity, homogeneity, degree, and pattern of contrast enhancement, suprasellar extension, mass effect on surrounding structures (hypothalamus, third ventricle, cavernous sinus), invasion of sphenoid bone, and/or sinus, evidence of peritumoral edema, and transtentorial invasion of sphenoid bone,

Results:

Enlargement of the pituitary gland with suprasellar extension was present in all cats. The pituitary gland had a variable appearance on precontrast T1W and T2W images. No characteristic signal patterns were identified on either T1W or T2W sequences. Contrast enhancement was nonuniform in all cats. The degree of contrast enhancement was mild in seven cats, moderate in five cats, and strong in three cats. Compression/invasion of the adjacent hypothalamus was suspected in all cases. A mass effect on the cavernous sinus and third ventricle was present in 13 cats. Invasion of the sphenoid sinus was not found; however there was a suspicion of invasion of the sphenoid bone in four cats. Mild peritumoral edema was present in four cats, and moderate edema in one cat. Transtentorial herniation was present in one cat, and mild cerebellar herniation in six cats. Histopathology confirmed the presence of a pituitary adenoma in two cases.

Discussion/Conclusion:

In conclusion, pituitary abnormalities were present in all 15 acromegalic cats but there was no consistent morphologic criterion. However, the pituitary gland in most cats had a heterogeneous appearance on both T1W and T2W images, with nonuniform contras enhancement. 


\section{COMPUTED TOMOGRAPHIC-BASED VERTEBRAL HEART SCALE IN AWAKE NORMAL CATS AND CATS WITH CARDIAC DISEASE}

C.R. Oliveira, K. Stadler, R.T. O’Brien. University of Illinois at Urbana-Champaign, Urbana

Introduction/Purpose:

Recently, the use of computed tomography (CT) in awake normal cats and cats with heart disease was described. The goals of this study were to establish the mean for CT-based VHS of awake normal cats and cats with heart disease, and to compare the findings with the VHS measured on radiographs.

Methods:

Eight cats confirmed by echocardiogram to be free of heart disease, 23 cats diagnosed with noncardiac-related diseases, and eight cats with echocardiographic confirmation of hypertrophic $(n=4)$, restrictive $(n=3)$, and arrythmogenic $(n=1)$ cardiomyopathies were scanned awake using a 16-slice CT machine and a positioning device (VetMouseTrap ${ }^{\mathrm{TM}}$ ). Left and right lateral, and dorsoventral or ventrodorsal radiographs were performed before or after the CT. All images were randomized. In radiographs, the VHS based on the width of the heart on a DV or VD view was measured according to a previously established method. For CT images, the dorsal plane was used to measure the width of the heart in its widest dimension following the same established method for VHS in radiographs. The numbers in millimeters $(\mathrm{mm})$ were plotted against the cranial aspect of T4 using the sagitta plane images. Normalcy of the data was confirmed with a Kolmogorov-Smirnov test. The mean, standard deviation (SD), and range were calculated for width of the heart in $\mathrm{mm}$ and VHS in number of vertebrae length for each group of cats. A chi-square test was used to compare the results between normal cats and cats with noncardiac-related disease. Since no difference was found, the results of these groups were analyzed together. The results of all measurements between noncardiac and cardiac cats were compared using independent sample $t$-test. A $P$ value $<0.05$ was considered significant.

Results:

The mean, SD, and range for width of the heart in $\mathrm{mm}$ were as follows: (1) radiographs: noncardiac group $=41.12(4.3,33-49)$, cardiac group $=56(5.8,48.3-65.1)$ (2) CT: noncardiac $=32.1(3.6,24.5-38.7)$, cardiac $=40.8(4,35.8-49.1)$. The result for VHS were as follows: (1) radiographs: noncardiac $=3.5 \vee(0.3,3-4.1)$, cardiac $=$ $4.7 \vee(0.35,4.2-5.2) ;(2)$ CT: noncardiac $=3.1 \vee(0.28,2.2-3.5)$, cardiac $=4.1 \vee(0.12$, 3.9-4.3). A statistically significant difference was found between all measurements in noncardiac $\times$ cardiac in both modalities $(P<0.0001, P<0.0003, P<0.0001$, and $P<$ noncardiac $\times$ cardiac

Discussion/Conclusion:

CT of awake cats allows evaluation of cats presented in heart failure that not always are stable for radiographic examination and therefore a new insight into cardiac disease can be gained. By measuring the VHS in the CT images, an objective evaluation of heart size is obtained and therefore a presumptive diagnosis of heart disease can be made. This can be of paramount importance in cats presented in respiratory distress as management of the heart failure can be initiated immediately. Our results indicate that CT-based VHS can the heart failure can be initiated immediately. Our results indicate that CT-based VHS can be used to objectively diagnose cardiomegaly with confidence and no overlap was found between normal cats and cats with heart disease. We propose an upper limit for CT-based cardiomegaly.

\section{DETECTION OF CANINE PULMONARY NODULES: COMPARISON OF CT,
COMPUTED RADIOGRAPHY, AND FILM-SCREEN RADIOGRAPHY}

K. Alexander, H. Joly, L. Blond, M.A. d'Anjou, M.E. Nadeau, J. Olive, G. Beauchamp. Faculté de médecine vétérinaire, Université de Montréal, St-Hyacinthe, Québec, Canada

\section{Introduction:}

Diagnostic imaging of the canine thorax for the staging of neoplasia is evolving. Computed tomography (CT) has become more widely available and digital and computed radiography (CR) have replaced film-screen (FS) radiography in many veterinary practices. However (CR) have replaced film-screen (FS) radiography in many veterinary practices. However,
few have compared these modalities in a clinical veterinary setting as a method to detect pulmonary nodules representing pulmonary metastasis. This study compared CT, CR, and FS radiography for the presence and number of pulmonary nodules in dogs.

Methods:

Client-owned dogs with a confirmed nonpulmonary primary neoplastic process were used both prospectively and retrospectively. All underwent CT and CR; those recruited prospectively also received FS radiographs. All images were evaluated in random order by two veterinary radiologists to form a consensus on the presence, total number, and size of pulmonary nodules. In addition, the lung was divided into six nonanatomically representative regions of the thorax (right cranial, middle, caudal; left cranial, middle, caudal) and the number and size of nodules in each region was evaluated. Additionally, for CT examinations, pulmonary atelectasis was graded semiquantitatively in each of the pulmonary regions. Nodule numbers and sizes were compared between all three modalities.

Results:

Twenty-one dogs underwent $\mathrm{CT}$ and $\mathrm{CR}$; nine also received FS radiographs. Positive/negative classification for the presence of nodules agreed between all three modalities in $8 / 9$ cases and between $C R$ and $C T$ in the remaining 12 cases. Reported confidence was highest for CT examinations (17/21 definitely positive or negative). CT detected the greatest $(P=0.002)$ total number of nodules; no difference was seen between $\mathrm{CR}$ and FS. The greatest number of nodules was seen in the right middle and both caudal regions, and only using CT $(P<0.0001)$. CT and CR detected a significantly smaller minimal nodule size than films when all nodules were considered together $(P=0.0007)$. CT detected a smaller than films when all nodules were considered together $(P=0.0007)$. CT detected a smaller
nodule size in both caudal and middle regions than radiographic modalities. Pulmonary atelectasis was significantly greater in the middle regions $(P<0.0001)$.
Discussion:

$\mathrm{CT}, \mathrm{CR}$, and FS performed similarly in determining the presence or absence of pulmonary nodules. Because the true nature of each pulmonary nodule could not be established, diagnostic accuracy of each modality should not be extrapolated from this study. Nonetheless, CT detected a greater number of smaller nodules, particularly in the caudal regions and overlying the heart, thereby potentially contributing to greater diagnostic confidence with this modality.

\section{EFFECT OF CONTRAST MEDIUM INJECTION DURATION ON THE CANINE PULMONARY ARTERIES PEAK ENHANCEMENT AND TIME-TO-PEAKENHANCEMENT USING DYNAMIC COMPUTED TOMOGRAPHY}

M. Makara ${ }^{1,2}$, M. Dennler ${ }^{3}$, K. Kühn ${ }^{3}$, K. Kalchofner ${ }^{4}$, P. Kircher ${ }^{3}$. ${ }^{1}$ Section of Diagnostic Imaging, Vetsuisse Faculty, University of Zurich, Switzerland, ${ }^{2}$ St. George's University, School of Veterinary Medicine, Grenada, ${ }^{3}$ Section of Diagnostic Imaging, ${ }^{4}$ Section Anaesthesiology, Vetsuisse Faculty, University of Zurich, Switzerland

\section{Introduction/Purpose:}

The goal of this project was to investigate the effect of contrast medium injection duration on pulmonary artery peak enhancement and time-to-peak enhancement.

Methods:

Fourteen dogs were allocated into one of seven predefined weight categories, each category contained two dogs. Dogs in each weight category were assigned to one of two different contrast medium injection protocols. In protocol $A$, a fixed injection rate of $5 \mathrm{ml} / \mathrm{s}$ was used. In protocol B, the iodine injection rate was calculated as follows: flow rate $=$ contrast volume/scanning duration $+10 \mathrm{~s}$. Time-to-peak enhancement and peak enhancement of the main left and right pulmonary arteries were measured on single-level, dynamic CT images for a fixed time of $30 \mathrm{~s}$. Rank correlation (Spearman) coefficients between injection duration and time-to-peak enhancement and between body weight and peak enhancement were calculated.

Results:

For group A, there was a significant negative correlation between patient weight and peak enhancement $(r=-0.94 ; P=0.005)$, while for group $\mathrm{B}$, there was no significant correlation $(r=-0.64$ and $P=0.18)$. There was a significant correlation between injection duration and time-to-peak enhancement for both groups (group A: $r=0.99 ; P=0.006$ and group B: $r=0.85 ; P=0.02$ )

Conclusion:

This study confirmed that the injection duration is a key feature in a CT angiography injection protocol. According to our results, an injection protocol with an injection duration adjusted to the scanning duration seems to be particularly suitable for veterinary applications where a population with great weight variability is studied.

\section{CANINE AND FELINE BICAVITY EFFUSIONS: A RETROSPECTIVE STUDY OF 164 CASES PRESENTING 2003-2008}

A.R. Mugford ${ }^{1}$, A. Parry ${ }^{2}$, M. Bevan ${ }^{1}$, P. Mantis ${ }^{1} .{ }^{1}$ Department of Veterinary Clinical Sciences, The Royal Veterinary College, University of London, ${ }^{2}$ Willows Referral Specialists Solihull Birmingham, UK

\section{Introduction:}

Bicavity effusions (BCE) have previously been associated with diagnoses of neoplasia and cardiovascular disease. The aim of this study was to evaluate the signalment, laboratory results, clinical signs, diagnoses, and outcome associated with animals with a diagnosis of $\mathrm{BCE}$ and to identify the most common diseases presented with BCE in the dog and cat. Materials and Methods:

One hundred and sixty-four cases of BCE admitted to the Queen Mother Hospital for Animals (QMHA) between March 2003 and June 2008 were identified from electronic patien records. Signalment data, presenting clinical signs, hematology, and biochemistry results and diagnosis were recorded for each BC patient and their control matched by species and date of admission. Outcome was recorded for BCE animals. All data were analyzed using chi-square tests for factors of significant for BCE. Logistic regression was then performed for factors, which remained significant when they were considered together with controls. Results:

During the study period, BCE represented $0.37 \%$ of the total hospital admissions. Of the 164 animals (99 dogs and 65 cats), most commonly observed diseases included cardiovascular diseases (46), neoplastic diseases (42), inflammatory/infectious diseases (20), renal diseases (7), non-neoplastic liver diseases (6), and pancreatitis (3). The majority of BCE animals were $8-12$ years old (mean age 93.3 months for BCE and 79.3 months for non-BCE animals). Male animals appear to be more affected by BCE. Of BCE patients: $56 \%$ survived to discharge (only $2 \%$ with resolution of disease), $50 \%$ were alive at last contact, $35 \%$ were euthanized during hospitalization, $4 \%$ of discharged patients were subsequently euthanized, and $9 \%$ died. There was no species predisposition. Among the feline population, there was no breed predisposition for BCE while for dogs, BCE was more likely to be seen in Labrador and spaniel dogs than terrier dogs.

Conclusions:

Dogs with BCE were most likely to have cardiovascular disease followed by neoplasia Cats with BCE were equally likely to have infectious/inflammatory disease as neoplasia. Cardiovascular disease was less likely than both in cats. BCE in cats and dogs should carry a very guarded prognosis since only $2 \%$ of cases were known to have recovered from the underlying disease process post discharge. 


\section{ELASTOGRAPHY AND CONTRAST-ENHANCED ULTRASONOGRAPHY OF FOCAL
LESIONS IN AN EXPERIMENTAL MODEL OF HEPATOCELLULAR CARCINOMA}

C.F. Carvalho, M.C. Chammas, V.M.R. de Lima, C.P.M.S. Oliveira. Instituto de Radiologia da Faculdade de Medicina da Universidade de São Paulo, São Paulo, Brazil

\section{Introduction/Purpose:}

Early detection of focal hepatic lesions is a challenge in clinical routine and becomes even greater in the presence of a diffuse parenchymal disease. Ultrasound is still the first choice for the screening of these diseases. Contrast-enhanced ultrasonography (CEUS) with perflubutane microbubbles improves the diagnostic accuracy to differentiate benign and malignan focal liver lesions. Hepatocelular carcinoma is the fifth most common cancer in human being and is a recognized complication of advanced nonalcoholic steatohepatitis (NASH).

This study aimed to evaluate the diagnostic performance of CEUS and elastography for early detection of malignant hepatic nodules in an experimental rat model ${ }^{1}$ of NASH.

Methods:

CEUS and elastography was performed in 10 normal adult Sprague-Dawley rats (contro group). B-mode and Doppler ultrasonography was performed weekly in 10 experimental rats with focal liver lesions until nodule detection with suggestive malignancy. Then the animals underwent elastography and CEUS, sacrifice, and assessment by microscopy. Tissue stiffness of the nodules on elastography was classified in negative (elastic strain) or positive (hard and no strain) comparing with surrounding liver parenchyma. Results:

In the early vascular phase with CEUS, wash in was significantly associated with malignancy with sensitivity of $71 \%$ and specificity of $100 \%$. In the late vascular phase, wash out patter was significantly associated with malignancy, sensitivity of $86 \%$, and specificity of $67 \%$. Elastograms of positive lesions showed area of high shear stiffness, which were indicative of malignancy confirmed on histology evaluation, with sensitivity of $100 \%$ and specificity of $90 \%$.

\section{Discussion/Conclusions:}

In the early and late vascular phase with CEUS were strongly suggestive of malignancy. Elastography provided promising perspectives for the assessment of malignancy of focal hepatic lesions in our sample. Both techniques allow making right diagnosis with high accuracy in an experimental rat model of NASH. Further studies are warranted to evaluate the effectiveness of this method for diagnosis of this disease in another species.

Reference:

1. Lima VMR, Oliveira CPMS, Alves VAF et al. A rodent model of NASH with cirrhosis, oval cell proliferation and hepatocellular carcinoma. J Hepatol 2008;49:1051-1061.

\section{ULTRASONOGRAPHIC MEASUREMENTS OF FELINE ADRENAL GLANDS IN CATS WITH HYPERTHYROIDISM}

A. Combes, E. Vandermeulen, L. Duchateau, K. Pèremans, S. Daminet, J.H. Saunders Faculty of Veterinary Medicine, Ghent University, Merelbeke, Belgium

\section{Introduction:}

Hyperthyroidism in cats is suggested to be associated with exaggerated responsiveness of the adrenal cortex. Little data are available about adrenal gland size in hyperthyroid of the

Methods: The adrenal glands of 27 hyperthyroid cats were examined
pared to the adrenal glands of 25 healthy cats of similar age.

pared to the

There was no difference in the shape of the adrenal glands between the two groups. They appeared as bean-shaped, well-defined, hypoechoic structures surrounded by a hyperechoic halo ( $35 / 50$ adrenal glands in healthy cats, 40/54 adrenal glands in hyperthyroid cats) Some cats showed more ovoid shape (11/50 adrenal glands in healthy cats, 11/54 adrena glands in hyperthyroid cats) or more elongated shape (4/50 adrenal glands in healthy cats $3 / 54$ adrenal glands in hyperthyroid cats). The adrenal glands of hyperthyroid cats presented $3 / 54$ adrenal glands in hyperthyroid cats). The adrenal glands of hyperthyroid cats presented
more often hyperechoic foci ( $9 / 27$ hyperthyroid cats, $2 / 25$ healthy cats). The adrenal glands were significantly larger in hyperthyroid cats than in the healthy cats (using the paired test), although there was an overlap in the range of measurements. In hyperthyroid cats, the adrenal length ranged from 7.9 to $19.4 \mathrm{~mm}$, the cranial height from 2.8 to $6.5 \mathrm{~mm}$, and the caudal height from 3.0 to $6.3 \mathrm{~mm}$. In healthy cats, the adrenal length ranged from 5.8 to $14 \mathrm{~mm}$, the cranial height from 1.5 to $5.9 \mathrm{~mm}$, and the caudal height from 2.3 to $5.2 \mathrm{~mm}$. The mean difference in length was $1.6 \mathrm{~mm}(\mathrm{SD}=0.4 ; P=0.0002)$, the mean difference in cranial height $0.6 \mathrm{~mm}(\mathrm{SD}=0.2 ; P=0.0039)$, and the mean difference in caudal height $0.5 \mathrm{~mm}(\mathrm{SD}=0.2 ; P=0.02)$

Discussion:

The enlargement of the adrenal glands in hyperthyroid cats was most likely associated with the increase in the functional adrenal capacity of the adrenal cortex documented in hyperthyroid cats. This bilateral homogeneous enlargement should be taken in account when a hyperthyroid cat is evaluated for adrenal disease or when hyperthyroidism and adrenal dishyperthyroid cat is evaluated for adrenal disease or when hyperthyroidism and adrenal dishyperadrenocorticism and hyperaldosteronism in cats presenting ultrasonographic bilateral adrenal moderate enlargement.

\section{ULTRASONOGRAPHIC REFERENCE VALUES FOR ADRENAL GLAND SIZE IN YORKSHIRE TERRIERS AND LABRADOR RETRIEVERS}

T. de Chalus ${ }^{2}, \underline{\text { A. Combes }}{ }^{1}$, A.S. Leperlier ${ }^{2}$, P. Pey ${ }^{1}$, L. Duchateau ${ }^{1}$, J. Saunders ${ }^{1} .{ }^{1}$ Ghen University, Ghent, Belgium, ${ }^{2}$ Ecole Nationale Vétérinaire d'Alfort, Maisons-Alfort, France

\section{Introduction/Purpose:}

An upper threshold of $7.4 \mathrm{~mm}$ for maximal adrenal diameter is commonly used to detect pituitary-dependent hyperadrenocorticism (PDH) using ultrasonography in dogs. However there is a substantial overlap between adrenal glands (AG) diameter of healthy dogs and those with PDH. The aim of the present study is to define the size of the AG in the York shire terrier and in the Labrador retriever, two breeds widely represented in the population suspected of hyperadrenocorticism, and, from these normal values, to determine the upper thresholds for the left and right height of the caudal pole in a longitudinal plane.

Methods:

Twenty-three healthy Labrador retrievers and 30 healthy Yorkshire terriers were included in this study. AG measurements of the length in a longitudinal plane, of the height at the cranial, and caudal pole in a longitudinal plane and transverse plane, and of the width at the cranial and caudal pole in a transverse plane, for a total of seven measurements per gland, were done on static images. A statistical analysis evaluated for each AG measurement the significance of the differences between the Labrador retrievers and the Yorkshire terriers. For each breed, the effects of age, weight, body length, body weight, and gender on each adrenal measurement were evaluated. Reference ranges including $95 \%$ of the dogs were calculated for each breed, each side (right or left), and each measurement, in order to determine the upper limits for the left and right AG in these two breeds.

Results:

New upper thresholds for the left and right height of the caudal pole in a longitudinal plane were determined: 7.9 and $9.45 \mathrm{~mm}$, respectively, for the Labrador retrievers and 5.4 and 6.7 $\mathrm{mm}$, respectively, for the Yorkshire terriers. All the measurements were significantly different between the 2 breeds and Labradors had larger AG measurements than Yorkshire terriers. between the 2 breeds and Labradors had larger AG measurements than Yorkshire terriers.
There was a significant relationship between height of the caudal pole and the age for both breeds. The relationship between the size of the AG and the weight was significant only for Yorkshire terriers. No relationship was found between body height, body length, and AG measurements. The gender had no effect on the measurements.

Discussion/Conclusions:

Yorkshire terriers have significantly smaller AG than Labrador retrievers and the age has a significant effect on the size of their glands, mainly on the caudal pole. The new uppe threshold values for the AG height at the caudal pole are $7.9 \mathrm{~mm}$ (left AG) and $9.45 \mathrm{~mm}$ (right $A G$ ) for the Labrador retrievers and $5.4 \mathrm{~mm}$ (left $A G$ ) and $6.7 \mathrm{~mm}$ (right $A G$ ) for the Yorkshire terriers. Further studies will determine the sensitivity and the specificity of these new upper thresholds as a diagnostic test for PDH.

\section{ENDOSCOPIC ULTRASONOGRAPHIC ASSESSMENT OF THE ESOPHAGEAL WALL IN DOGS}

P. Baloi, P. Kook, P.R. Kircher. Division of Diagnostic Imaging and Clinic for Small Animal Internal Medicine, Vetsuisse-Faculty, University of Zürich, Switzerland Introduction/Purpose:

The diagnostic approach for esophageal wall disease in dogs is currently limited to radiography, esophagoscopy, or rarely CT/MRI. However, none of these modalities is useful fo complete investigation of the esophageal architecture. In humans, endosonography (EUS) is employed for the detailed evaluation, as it provides highly accurate imaging of the wall and periluminal structures. In dogs, EUS has already been successfully used for the diagnosis of intrathoracic lesions, however no information exists on EUS characterizations of the esophagus and imaging of its five layers. Therefore, the goal of the present study was to endosonographically evaluate the different layers of the esophageal wall in healthy dogs and to compare them with previously provided histological results.

Methods:

Fourteen healthy beagle dogs (six intact males, eight intact females) with a mean body weight of $13.4 \mathrm{~kg}$, a median age of 2 years (1-5 years) were included in the study. Dogs were judged to be healthy based on the results of physical examination, $\mathrm{CBC}$, serum biochemistry, and urinalysis. EUS examination was performed in lateral recumbency under general anesthesia. A radial endosonoscope (UE 160-AL5 Radial endosonoscope, Olympus Schweiz AG, Volketswil, Switzerland) was used and images were stored on the attached ultrasound unit (Pro sound alpha 10, Aloka Holding AG, Switzerland). The endosonoscope was initially placed cranial to the cardia and then pulled up to the pharynx. To evaluate the wall layering and measure the thickness of the wall, images were obtained every $3 \mathrm{~cm}$ Images were taken with the probe directly in contact with the esophageal wall and with a water-filled balloon as a stand-off pad.

Results:

The esophageal wall thickness increased constantly from oral to aboral. In the proximal third, thickness amounted $2.19 \mathrm{~mm}(1.03-5.62 \mathrm{~mm})$ in the middle $2.15 \mathrm{~mm}(1.10-4.45$ $\mathrm{mm}$ ), and in the distal third $2.84 \mathrm{~mm}$ (range of 1.35-5.92 $\mathrm{mm}$ ). There was a significant difference between proximal third and distal third $(P<0.001)$. The results were simila with and without stand-off pad with only slightly smaller values when using the balloon. The wall layering appeared as five alternating hyperechoic and hypoechoic bands that were not constantly identified. There were three groups recognizable when assessing the identification of single layers: Group 1 was without visible layering, group 2 with three layers, and group 3 with all five layers visible. In $55.5 \%$ of all images, three layers could be identified but only in $15.7 \%$ all layers were visible. There was no significant difference in the visibility of the layering between the measurement with and without balloon.

Discussion/Conclusion:

Although the complete layering was not visible in all instances, the assessment of the architecture of the esophageal wall is possible with EUS. As opposed to studies in humans, we could not constantly identify all layers. The significant thicker wall in the caudal part of the esophagus does not correlate to histologic findings in standard textbooks of veterinary
histology. With this study, we provide information about normal EUS appearance of the esophagus. 


\section{NORMAL ULTRASONOGRAPHIC APPEARANCE OF THE PYLORUS AND CARDIA IN} FRENCH BULLDOGS

D.N. Rault ${ }^{1}$, L. Gatel ${ }^{2}$, E. Cauvin ${ }^{1}$, I. Testault ${ }^{3}$, L. Couturier ${ }^{1}$, P. Belli ${ }^{2} .{ }^{1}$ AZURVET, Re ferral Center in Veterinary Diagnostic Imaging and Neurology, F 06800 Cagnes-sur-Mer, ${ }^{2}$ Université de Lyon, VetAgro-Sup, F 69280 Marcy L'Etoile, ${ }^{3}$ ATLANTIA, Veterinary Hospital Center, F 44000 Nantes, France

Purpose:

Disorders of the pylorus and cardia have been reported to be common in brachycephalic dogs, especially French Bulldogs, in association with brachycephalic upper respiratory syndrome. The aim of the current study was to describe the normal ultrasonographic appearance of the cardia and pylorus in French Bulldogs and to assess the correlation between histologic and ultrasonographic architecture. The effects of sex, weight, age, and stomach filling were also investigated.

Materials and Methods:

A prospective study was performed in 35 French Bulldogs without respiratory or digestive abnormality. They weighed between 8 and $21 \mathrm{~kg}$, (mean $12.5 \mathrm{~kg}$ ), and were $0.8-10.5$ years of age (mean 5.2 years). There were 19 females and 16 males. The stomach was empty, moderately and markedly dilated in 21,6 , and 7 dogs, respectively. Ultrasound examinations were performed without sedation in dorsal recumbency using a microconvex transducer. The thickness of the pyloric wall $(P w)$, pyloric hypoechoic triangle $(P t)$, cardial $(C w)$, and antral wall $(A w)$, abdominal esophageal wall $(E w)$, and the esophageal diameter $(E d)$ were recorded. The pylorus was only measured when stretched out in an elongated position. Measurements were excluded if the pylorus was folded toward the stomach, thus protruding into the antral lumen. Measurements, effects of sex, weight, age, and stomach filling wer analyzed using Shapiro and Wilcoxon tests and linear regressions. One of the 35 French Bulldogs was euthanized after ultrasound because of a neurological disorder and underwent histological examination.

Results:

Measurement of the pylorus was obtained in $94 \%$ cases, of the cardia in $80 \%$ cases, and of the esophagus in $86 \%$ cases. Ultrasonographic layers were similar to histologic layers described in literature and confirmed in one case. Ultrasonographically, the cardia appeared as a "band like" thickening at the junction between the esophagus and stomach with hypertrophy of the muscularis. The pylorus was a short sphincter, bulging into the digestive lumen; the muscularis had a triangular shape. Aw ranged from 2 to $5.8 \mathrm{~mm},(\mathrm{~m}=$ $4.2 \mathrm{~mm}), P w$ from 5.9 to $8.4(m=7.5 \mathrm{~mm}), P t$ from 3 to $5.3 \mathrm{~mm}(m=4.3 \mathrm{~mm}), C w$ from $4.2 \mathrm{~mm}), P w$ from 5.9 to $8.4(m=7.5 \mathrm{~mm})$, Pt from 3 to $5.3 \mathrm{~mm}(m=4.3 \mathrm{~mm}), C w$ from
6 to $11.3 \mathrm{~mm}(m=8.7 \mathrm{~mm})$, Ed $8.4-15 \mathrm{~mm}(m=10.7 \mathrm{~mm})$, Ew from 3.8 to $8 \mathrm{~mm}(m=$ 6 to $11.3 \mathrm{~mm}(m=8.7 \mathrm{~mm}), E d 8.4-15 \mathrm{~mm}(m=10.7 \mathrm{~mm})$, Ew from 3.8 to $8 \mathrm{~mm}(m=$
$5.6 \mathrm{~mm})$. Distribution of measurements was normal $(P<0.05)$. There was no significant difference between males and females, and empty or full stomach (except for $A w-P<0.05$ ). No correlation was found between any measurements and age. Only a correlation between $P w$ and weight was noticed $(P<0.05)$

\section{Discussion/Conclusion:}

There was a good correlation between ultrasonographic and histologic appearance of the pylorus and cardia. Measurement of the cardia and pylorus was not possible in agitated dogs or those with a hiatus hernia. Adequate longitudinal sections of the pylorus were difficult to obtain because of the positioning, contraction or dilation of the stomach. The pylorus thickness in this section was less than $9 \mathrm{~mm}$. However, an oblique or folded pylorus artificially increased the thickness. Further investigations are needed to evaluate pyloric thickness in vomiting French Bulldogs with abnormal gastric emptying.

\section{ACTIVATION OF PERIPHERAL, BUT NOT CENTRAL, ALPHA-2-ADRENOCEPTORS WITH DEXMEDETOMIDINE INFLUENCES PERFUSION PARAMETERS MEASURED WITH CONTRAST-ENHANCED ULTRASOUND}

M. Leinonen ${ }^{1}$, F. Restitutti ${ }^{1}$, M. Raekallio ${ }^{1}$, R.T. O'Brien ${ }^{\star, 2}$, O. Vainio ${ }^{1} .{ }^{1}$ Department of M. Leinonen ${ }^{1}$, F. Restitutti 1 , M. Raekallio ${ }^{1}$, R.T. O'Brien ${ }^{\star, 2}$, O. Vainio ${ }^{1} \cdot{ }^{1}$ Department of Finland, ${ }^{2 *}$ Department of Veterinary Clinical Medicine, University of Illinois at UrbanaChampaign, IL

\section{Introduction/Purpose:}

Contrast-enhanced ultrasound (CEUS) has been used to detect organ perfusion in humans and dogs. No studies exist that compare the effect of dexmedetomidine (DEX) on perfusion of the kidneys and small intestine (SI) in dogs. MK-467 is a peripheral alpha $(\alpha)$-2-adrenoceptor antagonist that has shown to attenuate the early peripheral cardiovascular responses of $\alpha$ 2-adrenoceptor agonists in dogs. The purpose of this study was to compare the perfusion patterns in the kidney and SI in awake, sedated (with DEX and DEX combined with MK-467) healthy dogs.

Materials and Methods: Healthcare, Andover, MA). Qualitative (CEUS) was performed in six awake healthy labora- tory beagles. The animals were imaged both awake and after sedation with DEX $10 \mu \mathrm{g} / \mathrm{kg}$ IV and with DEX $10 \mu \mathrm{g} / \mathrm{kg}+$ MK-467 $500 \mu \mathrm{g} / \mathrm{kg}$ IV in a randomized, cross-over design with a 14-day wash out between treatments. Mechanical index was maintained at $0.05-0.07 \mathrm{de}-$ pending on depth of view and adjustable parameters were standardized. All dogs received multiple bolus injections $\left(0.05 \mathrm{ml} / \mathrm{kg}\right.$ ) of contrast medium (Sonovue $\left.{ }^{\circledR}\right)$ per imaging. A 1 to 2-min digital clip was recorded for each contrast injection and analyzed off-line (QLAB Philips). Standardized time-intensity curves were drawn from selected regions of interest in each organ and each recording separately. The perfusion parameters analyzed were arrival time (AT); time-to-peak intensity from the injection (TTPinj); baseline intensity (BI); peak intensity (PI); and wash-in rate (Wi). Analysis of variance with repeated measurements was used to compare the parameters of each organ, with the level of significance set as $P<0.05$. When $F$ values were significant, means were compared by the least-significant-difference method.

Results:

DEX significantly changed all parameters in the kidney $[\mathrm{AT} \uparrow(P<0.001), \operatorname{TTPinj} \uparrow(P<$ $0.001), \mathrm{PI} \downarrow(0.028), W i \downarrow(0.036)]$. Changes in AT $\uparrow(0.001)$, TTPinj $\uparrow(0.001), W i \downarrow(0.009)]$, but not PI(0.073) were found in the SI when compared to awake control animals. When DEX was compared to MK-467, significant change in AT $\downarrow(P=.018)$, TTPinj $\downarrow(P=0.006)$ in both kidney and $\mathrm{SI}$ and $\mathrm{Wi} \uparrow(P=0.008)$ in kidney only was found

Discussion/Conclusion:

These results indicate marked differences in the perfusion of the kidney cortex and $\mathrm{SI}$ assessed by CEUS in awake and DEX-treated animals. Concomitant use of MK-467 with DEX-maintained perfusion is similar to awaken dogs. Our results indicate that peripheral activation of $\alpha$-2-adrenoceptors influences both the timing and the intensity detected by CEUS. Thus, the effects of sedative agents on organ perfusion should be taken into consideration when interpreting the parameters measured by CEUS.

\section{MULTIDETECTOR-COMPUTED TOMOGRAPHY ENTEROGRAPHY USING WHOLE MILK AS A GASTROINTESTINAL CONTRAST AGENT IN DOGS}

D. Rodriguez ${ }^{1}$, M. Levy ${ }^{1}$, N. Rademacher ${ }^{1}$, P. Queiroz ${ }^{2}$, L. Gaschen ${ }^{1} .{ }^{1}$ Section of Diagnostic Imaging, ${ }^{2}$ Section of Veterinary Anesthesiology and Pain Management, Department of Veterinary Clinical Sciences, School of Veterinary Medicine, Louisiana State University, Baton Rouge, LA 70803

Introduction/Purpose:

Multidetector-computed tomography enterography (MDCTE) evaluates the gastrointestina system by using enteric contrast medium and intravenous-iodinated contrast medium to visualize enteric and extraenteric structures in people. MDCT angiography combined with low attenuation gastrointestinal contrast media such as whole milk allows detection of intestinal disease such as inflammatory bowel disease (IBD), ulceration, ischemia, and neoplasia in people. The purpose of this study is to evaluate whole milk as a gastrointestinal contrast agent in dogs, to compare it to traditional precontrast and postcontrast abdominal CT without milk, and to develop a protocol for MDCTE with bolus-tracking software.

Materials and Methods:

Five healthy purpose bred mongrel dogs were given $10 \mathrm{ml} / \mathrm{kg}$ of whole milk (4\% fat) 30 $90 \mathrm{~min}$ before induction of general anesthesia. Each dog was scanned with 16-slice CT scanner before and after administration of IV-iodinated contrast at standard doses using bolus-tracking software. A control group consisted of three MDCTA scans, pre- and post-IV contrast, without administration of milk. The images were subjectively assessed by three board-certified radiologists and graded for degree of intestinal distension: none (0), fair (1), good (2) and ideal (3), small intestinal wall enhancement (yes/no), and the ability to distinguish lumen from mucosa (yes/no). Mean attenuation values $(\mathrm{HU})$ of the pancreas outer intestinal wall, mucosa, and lumen of the intestines were calculated in each dog and compared between groups.

Results:

The small intestinal wall was enhanced in all evaluated groups, in the MDCTE group (milk) the mucosa was discriminated from the outer layers subjectively and by measuring mean attenuation values (HU). The lumen could only be differentiated from the mucosa in those dogs that received milk. Only the milk group postangiography had significantly different $(P<0.001)$ attenuation values $(\mathrm{HU})$ of all measured structures. Enteric distention was considered either good or ideal in the MDCTE group. Lack of enteric luminal distention created a pancreatic pseudolesion in one dog. Milk-related side effects were mild, and required no treatment and consisted of mild stool softening that lasted on average one day. required no treatment and

Discussion/Conclusions:
MDCTE resulted in superior intestinal wall enhancement, mucosal conspicuity, and either good or ideal lumen distension compared with MDCTA studies without milk. The use MDCTE protocols may have value for the diagnosis of intestinal diseases in dogs. Future studies comparing MDCTE studies to conventional ultrasound for detecting intestinal disease are necessary to establish whether the need for anesthesia and intravenous contrast agents are justified. 


\section{EVALUATING GASTROINTESTINAL WALL THICKNESS AND DIAMETER IN NORMAL DOGS USING COMPUTED TOMOGRAPHY}

S. Hoey ${ }^{1}$, R. Drees ${ }^{1}, X . Y u^{2} .{ }^{1}$ Department of Surgical Sciences, School of Veterinary Medicine, ${ }^{2}$ CALs Laboratory, Animal Science, University of Wisconsin-Madison, Madison, WI 53706

\section{Introduction/Purpose:}

Intestinal wall thickness and diameter can change with multiple disease processes, which can include infiltrative disease, inflammation, infection, edema, and infarction. Normal limits for gastrointestinal diameters and wall thickness have been established using radiography and ultrasonography, respectively. No information exists regarding the use of computed tomography in assessing intestinal wall thickness and diameter in normal canine patients. It was hypothesized that a reference range for canine gastrointestinal thickness and diameter could be formulated in relation to age and weight.

\section{Meight.}

In this retrospective study, the records of the Veterinary Medicine Teaching Hospital at UWMadison were searched for dogs that had no signs of gastrointestinal disease and were referred for abdominal CT studies between November 2007 and March 2011. Nineteen CT studies were identified that included both precontrast and postcontrast exams. The enhancement pattern and ability to visualize wall layering was noted. Measurements of multiple gastrointestinal segments for wall thickness and diameter were obtained using electronic calipers at 13 different anatomical locations. These values were assessed in relation to patient age and weight. Linear model and Kruskal-Wallis test were applied.

Results:

Detection of distinct wall layering of one or more anatomical section of the gastrointestina tract was present in 13 patients $(68.4 \%$ ) with stomach wall layering seen most consistently. Age was not significantly associated with intestinal diameter or wall thickness. With increase in weight, there was a significant increase in intestinal diameter and/or wall thickness of gastric fundus, body, pylorus, pyloric canal, duodenal cranial flexure, ascending duodenum jejunum, and ascending colon.

Discussion/Conclusions:

Gastrointestinal wall thickness and diameter increases with increase in weight as evaluated using postcontrast $\mathrm{CT}$ studies, thus $\mathrm{CT}$ evaluation of the abdomen can be a valuable choice for assessment of these parameters in dogs. Additional information about gastrointestina wall layering can also be obtained from postcontrast exams. Patient age did not affect wall layering can also be obtained from postcontrast exams. Patient age did not affect gastrointestinal wall thickness or diameter in this study. Further studies to evaluate the
application of CT assessment of gastrointestinal changes diameter in wall thickness in diseased animals are needed.

\section{RELATIONSHIP BETWEEN ULTRASONOGRAPHIC AND RADIOGRAPHIC FETAL BIOMETRY, MATERNAL, AND PREGNANCY-RELATED FACTORS, AND TIME TO PARTURITION IN QUEENS}

L. Gatel ${ }^{1}$, D.N. Rault ${ }^{2}$, K. Chalvet-Monfray ${ }^{1,3}$, S. Buff ${ }^{1} .{ }^{1}$ Université de Lyon, VetAgro-Sup - Campus Vétérinaire de Lyon, 1 Av Bourgelat F 69280 Marcy L'Etoile, ${ }^{2}$ AZURVET Referal Center in Veterinary Diagnostic Imaging and Neurology. Hippodrome, 2 Boulevard Kennedy F 06800 Cagnes-sur-Mer, France, ${ }^{3}$ INRA, UR 346 Epidémiologie Animale, F-63122 SaintGenès-Champanelle, France

Purpose:

The aim of this study was to create a model to predict more accurately the timing of the onset of parturition in queens, using radiographic and ultrasonographic measurements of fetal femoral length (FL) and biparietal diameter (BPD). The effects of litter size, weight, and age of the queen on the accuracy of the prediction were also investigated.

Materials and Methods:

A prospective study was performed in 24 purebred queens of 11 different breeds, in normal body condition. Pregnant queens with aborted or malformed fetuses were excluded from the study. Ultrasonographic and radiographic examinations were performed without sedation, during the second half of pregnancy, once to three times in each queen. Ultrasonographic measurements were performed on each fetus using a microconvex transducer; the maximal FL and the transversal BPD were recorded. Digital radiographs were obtained and reviewed to determine the litter size and to measure the FL and the BPD. The parturition time was estimated using linear mixed-effects models on R Package, version 2.5.1.

Results:

The best multiple linear regression included ultrasonographic measurement of the femora length $(F L)$, the weight of the queen before pregnancy $(W)$, the litter size $(L S)$, and the age of the queen $(A)$. All these parameters were significant $(P<0.01)$. The formula to predic the number of days to parturition $(Y)$ was $Y=37.864-0.193 \times F L+1.227 \times W$ $0.615 \times L S-0.832 \times A$. The $70 \%$ prediction interval was $Y \pm 1.6$ days. Models using the FL provided a more accurate prediction of the onset of parturition than models using BPD. Time to parturition significantly increased with the weight of the queen before mating $(P<$ $0.01)$. Time to parturition was shorter when the femur was longer $(P<0.01)$, the litter size larger and when the queen was older $(P<0.01)$. No correlation was found between time to parturition and breed or wither height.

Discussion/Conclusion:

Radiography is essential to count the number of fetuses but it is not accurate to predict the time to parturition. Combining ultrasonographic measurement of the fetal femoral length, the queen's weight and age, and the number of fetuses, provided a model of prediction of the onset of parturition in the second half of pregnancy. This study shows that models using the FL provide a more accurate prediction of the onset of parturition than previous models the FL provide a more accurate prediction of the onset of parturition than previous models
using BPD in queens. A larger population of cats should now be investigated to allow for discrimination between the specific effects of breed and female size. Further investigations may also allow quantification of the effects of oversize, obesity, or illness.

\section{MINIMALLY INVASIVE ULTRASOUND-GUIDED RETRIEVAL OF MIGRATING} SUBLUMBAR PLANT AWNS IN EIGHT DOGS

D. Della Santa. Clinica Veterinaria Mugello, via Provinciale 3D, San Piero a Sieve (FI), Italy

Introduction/Purpose:

Paraspinal muscle infection and abscessation in the lumbar region is a relatively common condition often induced by the migration of plant awns into the sublumbar region after having been inhaled previously. In such cases, the treatment of choice is surgical exploration of the sublumbar musculature through a transabdominal approach. The aim of the present pape is to report the results of ultrasound-guided nonsurgical retrieval of sublumbar migrating plant awns in dogs.

Methods:

In this retrospective study were included dogs with clinical signs consistent with foreign body-induced paraspinal infection where a migrating plant awn could be clearly identified sonographically in the sublumbar region and an ultrasound-guided nonsurgical retrieval procedure with Hartmann forceps was attempted. Results:

In the period 2008-2011, eight cases fulfilled the inclusion criteria and were included in the study. In all cases, the foreign body was located at the level of the mid-lumbar spine either in a iliopsoas muscle (6/8) or retroperitoneal fat (2/8). In all cases, the Hartmann forceps were inserted in the subcutaneous tissue of the dorsal lumbar region and then directed ventrally or cranioventrally pointing toward the sublumbar foreign body. In $2 / 8$ cases, ultrasound allowed identification of a fistulous tract connecting the sublumbar foreign body and the subcutaneous tissue that could be used as a pathway for the Hartmann forceps without the need of neous tissue that could be used as a pathway for the Hartmann forceps without the need of extensive tissue dissection. In $5 / 8$ cases, more extensive tissue dissection was necessary
because of the absence of a fistulous tract suitable to be used for reaching the foreign body; in such cases, blunt dissection was performed with Hartmann forceps. In the last case, a trocar was used to dissect the soft tissues and used as a port to insert the Hartmann forceps in close proximity of the foreign body. Finally, in all cases, the foreign bodies were grasped and retrieved. In $6 / 8$ cases, the plant awn was completely retrieved and the condition did not
recur. In one case, one foreign body was successfully retrieved, but the condition recurred because of a second plant awn (not detected during the first procedure) that was retrieved with a second procedure performed with the same technique; no recurrence was observed after the second procedure. In the last case, the foreign body was divided into two parts and only one of them could be retrieved because of cranial displacement of the second fragment during the procedure. No harmful complication occurred during the procedures, nor was observed at follow-up examinations.

observed at follow-up examina

Discussion/Conclusions: tion, alternative to standard surgical operation, for treatment of dogs with sonographically confirmed sublumbar foreign bodies.

\section{RELATIONSHIP BETWEEN COMPUTED TOMOGRAPHIC SIGNS AND ARTHROSCOPIC FINDINGS IN DOGS WITH MEDIAL CORONOID PROCESS DISEASE: WORK IN PROGRESS}

E. Kulendra, M. Tivers, J. Grierson, C.R. Lamb. The Royal Veterinary College, University of London, UK

\section{Introduction:}

Computed tomography (CT) is routinely used to examine the elbows of dogs with suspected medial coronoid process (MCP) disease; however, there is poor correlation between CT signs and arthroscopic findings in these patients. The aim of this study is to elucidate the relationship between CT signs and arthroscopic findings in dogs with MCP disease.

Methods:

Prospective cross-sectional study of dogs having arthroscopic treatment for MCP disease. The appearance of the MCP, with emphasis on fissures and fragmentation, was compared in preoperative and postoperative $\mathrm{CT}$ images and the differences correlated with the surgical reports.

Results:

Fifteen dogs (22 elbows) have been studied to date. Median age at arthroscopy was 13 months (range 7-96 months). There were 13 males and 2 females. The most frequent breed was Retriever $(n=6)$. Fragments were removed arthroscopically from the MCP in $18(82 \%)$ elbows; the remaining four elbows had chondromalacia affecting the MCP. Preoperative CT images showed signs of fissures affecting the MCP or articular calcified bodies erative $\mathrm{CT}$ images showed signs of fissures affecting the MCP or articular calcified bodies
compatible with fragments in $14(78 \%)$ elbows in which fragments were subsequently found arthroscopically. Single fragments were evident in CT images in five (23\%) and multiple fragments were evident in five (23\%) elbows. Neither fissures nor fragments were evident in CT images of elbows with chondromalacia only. Comparison of preoperative and postoperative CT images demonstrated complete removal of fragments in two (11\%) instances, reduced number of multiple fragments in five $(28 \%)$ instances, and reduced size of fragments in four $(22 \%)$ instances. Fragments were visible only in postoperative CT images in three $(14 \%)$ $(22 \%)$ instances. Fragments were visible only in postoperative CT images in three (14\%)
instances. Evidence of debridement of subchondral bone was evident in postoperative CT instances. Evidence of debridem
images in $15(68 \%)$ instances.

Conclusions:

Arthroscopy did not result in removal of all suspected fragments identified by CT, and some lesions suspected to be fragments were reduced in size rather than removed. Each of these findings suggests that suspected fragments identified by $\mathrm{CT}$ are not always visible arthroscopically, for example, because they are nondisplaced, covered by cartilage or beyond the range of the scope. The occurrence of fragments only in some postoperative CT images suggests that the act of debriding the MCP can itself cause fragmentation.

Reference:

This study is funded by Petsavers. 


\section{ANALYSIS OF THE CAUSES OF LAMENESS IN LARGE AND GIANT BREED DOGS OBSERVED DURING THE YEARS 1990-2007 IN THE EAST REGION OF POLAND: RETROSPECTIVE RESEARCH} A. Lojszczyk-Szczepaniak. University of Life Science in Lublin, Laboratory of Radiology and

\section{Introduction:}

The presented research is a continuation of research from the 1980s on the occurrence of several diseases in young dogs during the period of their skeletal maturation. The research was published in Veterinary Radiology (1988, volume 29) by Professor Stanislaw Koper. This period was characterized by the lack of necessary food for dogs both from the perspective of quantity and quality. From the beginning, the 1990s dog food produced by many internationally known companies appeared. In these conditions, an excessively calorific diet, especially for dogs in the period of skeletal maturation, became highly probable.

Methods:

The study presents radiographically diagnosed causes of lameness in 954 dogs of both sexes, mainly large and giant breed up until their first year of life. The dogs underwent clinical and radiographic examination, which was conducted in the years 1990-2007. Dogs were divided into groups encompassing the type of disease diagnosed radiographically. The following diseases were noted in the subsequent 3-year periods: secondary nutritional hyperparathyroidism (SNHPT), panosteitis eosynophilica (PE), osteochondrosis (OC_taking into account all the known clinical forms). Hypertrophic osteodystrophy (HOD) was also

taken into

The research confirmed that the most frequently burdened with lameness dog breeds were German Shepherds: 358 dogs $(37.53 \%)$ and Rottweilers: 90 (9.43\%). Panosteitis was ac knowledged as the main cause of lameness. The disease mainly affected German Shepherds. The frequency with which PE occurred in the years 1990-1992 was 17 cases from 48 patients $(35.42 \%)$ and its frequency decreased by degrees to the level of 58 cases from 220 patients $(26.36 \%)$ in the 2005-2007. Similarly, a decline in SNHPT and osteochondrosis was observed, with the exception of the early period of the 1990s, in which a slightly large number of cases of OC $(17,13.49 \%)$ were noted. As a disease unit rickets occasionally occurred. There was a high percentage of dogs with a few diseases recognized at the same time. The most frequently confirmed diseases were HOD and SNHPT, primarily in German Shepherds.

Discussion: Diseases from the period of skeletal maturation in dogs are conditioned by genetic and environmental factors, the latter primarily from their diet. The results of the conducted obser-
vations prove the decline of the examined diseases, which constitutes a fairly advantageous condition for developing breeding programs in eastern Poland. The insignificant growth of OC cases at the beginning of the 1990s was caused by the lack of sufficient knowledge concerning feeding dogs with manufactured dog food. An excess of energy connected with an excessively calorific diet was highly probable.

\section{COMPUTED TOMOGRAPHIC DEMONSTRATION OF THE CALCANEO-CENTRAL} TARSAL BONE ARCHITECTURE IN DOGS

G. Galateanu ${ }^{1}$, I. Aizenberg ${ }^{2}$, T.B. Hildebrandt ${ }^{1}$, D. Apelt ${ }^{3} .{ }^{1}$ Leibniz Institute for Zoo and Wildlife Research, Berlin, Germany, ${ }^{2}$ Hebrew University Veterinary Teaching Hospital, BeitDagan, Israel, ${ }^{3}$ Tierärztliche Klinik für Klein- und Heimtiere, Berlin, Germany

\section{Introduction/Purpose:}

The mechanism of central tarsal bone (CTB) fractures is not fully understood and additiona information regarding its etiology is necessary. While in racing dogs, these fractures are mostly defined as stress fractures of the CTB body, in nonracing dogs are mainly described fracture/luxations of the CTB concomitant with a fracture/avulsion of the plantar process of the CTB ( $\left.\mathrm{PP}_{\text {СтB }}\right)$. Therefore, the role of the $\mathrm{PP}_{\mathrm{CTB}}$ was investigated, though the assessment of pathological changes at this level can be challenging. Clinical orthopedic evaluation, standard radiographic examination, and even special radiographic views are often insuffistandard radiographic examination, and even special radiographic views are often insuffi-
cient to rule out pathology at this site. The main disadvantage of conventional radiographic techniques is the projection of a three-dimensional structure in a two-dimensional plane resulting in superimposition of various and irregular bony structures. We therefore used 128-slices high-resolution computed tomography (CT) to achieve better evaluation of the $\mathrm{PP}_{\mathrm{CTB}}$ and its spatial relationship with the TBs.

\section{Methods:}

Images resulting from CT examinations of 52 tarsal joints belonging to 27 dogs (24 dogs of 18 breeds and three mixed breeds), among them, two active police German Shepherd dogs, were analyzed. The position of the $\mathrm{PP}_{\mathrm{CTB}}$, its shape, cortical features, and articular surface with the calcaneus were investigated

Results:

Thanks to its technical advantages, we were able to visualize, using the $\mathrm{CT}$, that in all studied joints, the proximolateral part of the PP the calcaneus is juxtaposed to the CTB. In joints under stress (working dogs), high-resolution CT images revealed cortical remodeling of the $\mathrm{PP}_{\mathrm{CTB}}$.

\section{Discussion/Conclusions:}

Based on tarsal architecture and spatial relationship between the calcaneus and the $\mathrm{PP}_{\text {CTB }}$ demonstrated in this study, we suggest that at least some of CTB fractures in dogs either start or are induced at the level of the $\mathrm{PP}_{\text {СтB }}$ as a result of the impingement forces exercised by the calcaneus on the $\mathrm{PP}_{\mathrm{CTB}}$. We postulate that during some movement phases (i.e., adduction) the PP adduction) the $\mathrm{PP}_{\mathrm{CTB}}$, and, respectively, the rest of the $\mathrm{CTB}$, have to absorb more impact shocks due to calcaneal compressive effect on a wider, shock-absorber surface of the
$\mathrm{PP}_{\text {CTB }}$. This complementary mechanism may explain the pathogenesis of CTB fractures in racing, working and nonworking dogs.

\section{COMPUTED TOMOGRAPHY OF THE SCAPULOHUMERAL JOINT AND PERIARTICULAR REGION: A RETROSPECTIVE STUDY OF FINDINGS IN 75 DOGS PRESENTING WITH THORACIC LIMB LAMENESS}

T.W. Maddox ${ }^{1}$, C. May ${ }^{2}$, B. Keeley ${ }^{2}$, J.F. McConnell ${ }^{1} .{ }^{1}$ Small Animal Teaching Hospital, University of Liverpool, ${ }^{2}$ North West Surgeons, Sutton Weaver, Cheshire, UK

Introduction/Purpose:

Computed tomography (CT) is increasing employed as an aid to the diagnosis of thoracic limb lameness; however, few studies have assessed the prevalence and significance of the lesions that may be identified by this imaging modality in the scapulohumeral (shoulder) joint and periarticular structures.

Methods:

Records of two referral veterinary clinics were searched for client-owned dogs with lameness in one or both thoracic limbs that had CT examination of both shoulder and elbow joints. Dogs were excluded if they had not undergone concurrent full orthopedic examination (including lameness grading and localization of the site of lameness). All CT images were reconstructed using soft-tissue and bone windows, multiplanar reconstruction was employed when required. Images were evaluated for the presence of articular and periarticular elbow and shoulder pathology in both thoracic limbs. Clinical records were interrogated for signalment data, the results of orthopedic examination, and further diagnostic tests (including arthroscopy) and case outcome. Associations between the presence of shoulder lameness and CT findings were examined using multilevel logistic regression.

Results:

In total, 80 dogs were identified as having undergone shoulder and elbow CT examination, five were excluded as they had not undergone orthopedic examination. The study population consisted of 27 Labrador retrievers, 9 spaniel breeds, 7 Border collies, and 20 other breeds. Thirty-six dogs had lameness attributable to the elbow joint, 16 to the shoulder joint, 17 to both shoulder and elbow, with the remainder having no localizable lameness. Abnormal CT findings were documented in 31 dogs $(41 \%)$. A CT diagnosis of humeral head osteochondrosis was made in 7 dogs $(9 \%)$ and 15 dogs $(20 \%)$ had evidence of scapulohumeral osteoarthritis. Twenty-five dogs ( $33 \%)$ had evidence of mineralization of the supraspinatus ( $n$ $=20)$, infraspinatus $(n=6)$, biceps $(n=4)$, or subscapularis $(n=2)$ musculature/tendons. Only six of these animals had evidence of ipsilateral shoulder pain and periarticular mineralization was not associated with lameness localizable to the shoulder $(P=0.28)$. Periarticular mineralization was not significantly associated with any breed $(P=0.37)$. The diagnosis of osteochondrosis by CT was the only lesion significantly associated with shoulder lameness $(P<0.01)$. Twelve cases underwent shoulder arthroscopy and for 10 cases the CT and arthroscopic diagnosis agreed; osteochondrosis $(n=6)$, articular fracture $(n=1)$, shoulder osteoarthritis $(n=1)$, and no detectable lesions $(n=2)$. The remaining two cases had cartilage changes not detected by CT examination.

Discussion/Conclusion:

The prevalence of abnormal CT findings relating to the scapulohumeral joint is relatively high in dogs with thoracic limb lameness, but of these only osteochondrosis, lesions appea to have a significant association with lameness localized to the shoulder. Mineralization of periarticular musculature/tendons appears common but would seem to have little clinical significance. 


\section{ULTRASOUND GUIDANCE FOR THE FEMORAL NERVE BLOCK IN CATS: AN IMAGING STUDY}

P. Haro Alvarez ${ }^{1}$, F. Gil ${ }^{2}$, F.G. Laredo ${ }^{1}$, M.D. Ayala ${ }^{2}$, E. Belda ${ }^{1}$, M. Soler ${ }^{1}$, A. Agut ${ }^{1}$ ${ }^{1}$ Department of Medicine and Surgery, ${ }^{2}$ Department of Anatomy and Compared Pathological Anatomy, University of Murcia, Spain

\section{Introduction/Purpose:}

The ultrasound (US)-guided blockade of the femoral nerve (FN) has been described in dogs at the level of the femoral triangle. To the author's knowledge, information regarding the US location and blockade of the FN has not been documented in the cat. The aim of this study was to develop a technique for a US-guided dorsal approach to the FN in cats.

\section{Materials and Methods:}

Anatomic study. Four fresh adult feline cadavers were employed for dissection and cross sectional study of the FN. Imaging study. Another four adult feline cadavers were used to develop the intended FN approach. The cats were positioned on lateral recumbence and scanned from $\mathrm{L} 2$ to the sacrum using a $13 \mathrm{MHz}$ linear transducer. The probe was placed perpendicular to the spine with the mark in a dorsal position. One milliliter of blue ink was injected around the FN to confirm the accuracy of the FN location. Two of the cadaver were immediately dissected; the other two were frozen and cut on transverse cryosections to study the spread pattern of the ink. In other four cadavers, $1 \mathrm{ml}$ of iodinated contrast $(150 \mathrm{mgl} / \mathrm{ml})$ was injected around the FN to verify the feasibility of the studied approach Then, orthogonal radiographs and a CT scan of the lumbar spine (L2-S3) were performed to assess the location of the needle and the spread of the contrast.

Results:

Anatomic study: The FN was located between the iliacus and the psoas major muscles (iliopsoas complex). Imaging study. The anatomic landmarks used to localize the FN were the dorsal process of L6-L7 lumbar vertebrae and the cranial border of the wing of the os ilii. The iliopsoas complex had an oval shape and a hypoechoic appearance related to the ilii. The iliopsoas complex had an oval shape and a hypoechoic appearance related to the epaxial muscles and retroperitoneal fat. The US appearance of the FN was as a hypoechoic an in plane technique between the transverse process of L6-L7. The ink injected was observed staining the $\mathrm{L} 5$ and $\mathrm{L} 6$ nerve roots, the $\mathrm{FN}$, the quadratus lumborum, psoas major, psoas minor, and iliacus muscles in all cases. A cranial and caudal distribution of the in from the site of injection was also observed. The radiographs and CT scans confirmed the location of the needle between the L6-L7 transverse process and within the iliopsoas complex in all cases. The contrast media was observed spreading at the level of the ventra part of the vertebral body of $L 5$ in one case (1/4). A small amount of contrast was observed outside the iliopsoas into the retroperitoneal space in three cases $(3 / 4)$

Conclusions:

The US-guided dorsal approach may be useful to localize and block the FN in cats. Further studies are necessary to prove its feasibility in a clinical context.

Reference:

1. Echeverry DF, Gil F, Laredo F, et al. Ultrasound-guided block of the sciatic and femoral nerves in dogs: a descriptive study. Vet J 2010; 186:210-215.

\section{ASSESSMENT OF A VENTRAL ULTRASOUND-GUIDED SUPRAINGUINAL APPROACH TO BLOCK THE FEMORAL NERVE IN THE DOG}

D.F. Echeverry ${ }^{1}$, A. Agut $^{2}$, F. Laredo ${ }^{2}$, E. Belda ${ }^{2}$, M. Soler ${ }^{2}$, F. Gil ${ }^{3} .{ }^{1}$ Department of Animal Health, Faculty of Veterinary Medicine and Zootechnic, University of Tolima, Ibague, Colombia, ${ }^{2}$ Department of Medicine and Surgery, ${ }^{3}$ Department of Veterinary Anatomy and Embryology, Faculty of Veterinary, University of Murcia, Spain

\section{Introduction/Purpose:}

The anesthetic blockade of the femoral (FN) and sciatic nerves has demonstrated to provide similar analgesia than the epidurals with a lower incidence of adverse effects. The success of this technique is highly dependent on the adequate location of target nerves. In a recent study, ultrasound (US) techniques of nerve location were optimal to block the sciatic nerve in the dog. However, a suboptimal blockade of the FN (50-62.5\%) was reported when using a ventral US-guided infrainguinal approach in dogs. A new ventral US-guided suprainguinal approach to block the FN is evaluated in this study. The ultrasonographic characteristics of the FN and the feasibility and accuracy of this approach are investigated in cadavers and the $\mathrm{FN}$ and the feasi Materials and Methods:

Materials and Methods:

Anatomical nerve study: The evaluation of the anatomical characteristics of the FN required to obtain an optimal acoustic window was performed in four intact dog cadavers. The dissection of the FN was carried out in two cadavers, and the other two were frozen and cut to perform a cross sectional study. In the "in vitro" part of the study the left and right FN of five intact cadavers were identified by US. The US probe (13 MHz linear array) was orientated perpendicular to the midline and slightly cranial to the inguinal nipple. From this point, the perpendicular to the midline and slightly cranial to the inguinal nipple. From this point, the
probe was directed cranially, trying to trace the projection of the nerve on the abdomen. The probe was directed cranially, trying to trace the projection of the nerve on the abdomen. The
ultrasonographic features of the FN and related structures were described. The accuracy of the US-guided nerve localization was demonstrated by injecting a staining solution around the nerve. In this phase, a success FN block was defined as the staining of the nerve in a length $\geq 2 \mathrm{~cm}$. In the "in vivo" ultrasound-guided nerve blockade, the left and right FN were located by US in five experimental Beagles sedated with medetomidine $(10 \mu \mathrm{g} / \mathrm{kg}$ given IM). When the needle was seen close to the FN lidocaine $2 \%(0.3 \mathrm{ml} / \mathrm{kg})$ was injected given IM). When the needle was seen close to the FN lidocaine $2 \%(0.3 \mathrm{ml} / \mathrm{kg})$ was injected
around the nerve. The spreading of the local anesthetic was observed during the injection. At the end of the experience, atipamezole $(10 \mu \mathrm{g} / \mathrm{kg}$ given IM) was administered to reverse sedation. In this phase, a successful FN block was confirmed by the presence of motor and proprioceptive deficit, and inability to move the hindlimb. The dogs were re-evaluated 12 and $24 \mathrm{~h}$ after the blockades.

Results:

The FN was observed as a hypoechogenic rounded structure surrounded by a marked hyperechogenic thick ring, located within the iliopsoas muscle body on transverse scans. The FN was accurately located by US in all the cases. This was confirmed in vitro by the staining of the FN in a length superior to $2 \mathrm{~cm}$, and in vivo, by the presence of a successful FN block. No neurological complications were observed.

\section{Discussion/Conclusion:}

This ventral US-guided suprainguinal approach may be a feasible and accurate method to approach and block more efficiently the FN in the dog.

\section{MAGNETIC RESONANCE IMAGING FEATURES OF DISCOSPONDYLITIS IN DOGS}

I. Carrera ${ }^{1}$, R. Gonçalves ${ }^{2}$, F. McConnell ${ }^{2}$, M. Sullivan ${ }^{1}$. ${ }^{1}$ Faculty of Veterinary Medicine, University of Glasgow, Glasgow, ${ }^{2}$ Small Animal Teaching Hospital, University of Liverpool, Neston, UK

Introduction:

The diagnosis of discospondylitis is based on clinical signs, diagnostic imaging, and laboratory results. To date, radiography has been the imaging technique most widely used. This retrospective study aimed to describe the magnetic resonance imaging (MRI) findings observed in dogs with confirmed discospondylitis and evaluate the advantages and limitations of MRI in the management of this condition.

\section{Materials and Methods:}

The inclusion criteria comprised complete clinical history and neurological examination, radiographs, and MRI study of the region of the vertebral column affected, including in all cases sequences precontrast and postcontrast T1-weighted sequences: urine, blood, and/or intervertebral disc cultures, and follow-up of the progression of the clinical signs in response to antibiotic treatment.

to antibio

Thirteen dogs were included with confirmed discospondylitis. In total, there were 17 sites of discospondylitis. Eleven $(81.1 \%)$ of the dogs had spinal pain for more than 3 weeks and a variable degree of neurological signs. Two dogs had spinal pain and ataxia for 4 days. In MRI images, there was always involvement of two adjacent vertebral endplates and its associated intervertebral disc. The involved endplates and adjacent bone marrow were T1W hypointense with hyperintensity in fat suppression techniques. All dogs had contrast T1W hypointense with hyperintensity in fat suppression techniques. All dogs had contrast
enhancement of endplates and paravertebral soft tissues. The intervertebral discs were
hyperintense in T2W and fat suppression and characterized by contrast enhancement in 15 hyperintense in T2W and fat suppression and characterized by contrast enhancement in 15
sites $(88.2 \%)$. Endplate erosion was present in 15 sites (88.2\%) and was associated with T2-hypointense bone marrow adjacent to it. In two sites (11.8\%), endplate erosion was no seen in MRI or radiography. The vertebral bone marrow in these cases was hyperintense in $\mathrm{T} 2 \mathrm{~W}$ and in fat suppression sequences, and also showed contrast enhancement. Epidural extension was conspicuous in postcontrast images in 15 cases $(88.2 \%)$. Spinal cord compression was present in 15 cases $(88.2 \%)$, which presented clinical evidence of neurological signs. Subluxation was present in two sites (11.8\%).

Discussion/Conclusion:

MRI shows characteristic features of discospondylitis and allows the recognition of the exact location and extension (to the epidural space and paravertebral soft tissues) of the infection. Furthermore, MRI increases lesion conspicuity in early discospondylitis that may not be visualized by radiography.

\section{FLEXED SAGITTAL MRI FOR EVALUATION OF THE OCCIPITAL BONE AND FORAMEN MAGNUM}

R. Dennis. Centre for Small Animal Studies, Animal Health Trust, Lanwades Park, Kentford, Newmarket, Suffolk, UK

\section{Introduction/Purpose:}

Chiari-like malformation (CM) due to changes in the occipital bone is a well-recognized deformity in several small dog breeds, notably the Cavalier King Charles spaniel, in which it seems to be almost ubiquitous. The morphological abnormality results in a mismatch between cerebellar size and caudal fossa volume resulting in foramen magnum "crowding" or cerebellar herniation. It is thought that this causes disturbance of CSF flow, leading to syringomyelia (SM). MRI is widely used for CM/SM diagnosis and screening, a midline sagittal T1- or T2-weighted image being used to assess CM.

\section{Magittal T1- or T2-weighted}

In August 2010, the protocol for MRI of CKCS undergoing investigation of suspected CM/SM at the Animal Health Trust was extended to include a moderately flexed, sagittal T2W scan as well as the usual extended sagittal scan. Dogs are scanned supine, and following the initial scans, the head is flexed to about $125^{\circ}$ at the occipito-atlantal junction and held in place using small foam pads and sticky tape. After a further three-plane localizer, a second sagittal T2W scan (+I- a sagittal T1W scan) is performed. Flexed sagittal scans have also been performed on a number of other dogs of varying breed, size, and conformation for comparison.

Results:

The appearance of the occipital bone varies markedly between breeds. In larger dogs, the portion of the occipital bone, which forms the dorsal margin of the foramen magnum, is slightly thickened, forming a "toe" with a distinct, hyperintense medullary cavity. The bone is easily distinguished from the dorsal ligaments of the joint, which are folded when the head and neck are moderately extended. In smaller dogs, the occipital bone is thinner and lacks the marrow-filled "toe." It appears as a narrow band of signal void intensity, which cannot be differentiated from the ligaments. On flexion, the folded ligaments flatten out and their poin of attachment to the occipital bone is more easily identified. In the majority of CKCS and in some other small breed dogs, marked increase in height of the foramen magnum is evident on flexion. This shows that the cerebellar vermis is usually herniated, rather than following the apparent contour of bone.

Discussion/Conclusions:

The study has shown that similarity of signal intensity between the occipital bone and the dorsal ligaments of the occipito-atlantal joint may lead to incorrect assessment of occipita bone shape and underestimation of the height of the foramen magnum and the degree of cerebellar herniation on scans obtained with the dog's head and neck in moderate extension. This has an impact of research into CM/SM where the foramen magnum diameter is measured on sagittal MR images. The flexed sagittal position is therefore recommended for clinical diagnosis and morphometric studies. It also shows more accurately whether or not cisternal puncture for CSF collection is hazardous. 


\section{CRANIAL THORACIC SPINAL STENOSIS IN LARGE BREED DOGS}

P.J. Johnson ${ }^{1}$, L. De Risio ${ }^{1}$, J.F. McConnell ${ }^{2}$, A.H. Sparkes ${ }^{1}$, R. Dennis ${ }^{1}$, A. Holloway ${ }^{1}$. ${ }^{1}$ Animal Health Trust, Kentford, Newmarket, UK, ${ }^{2}$ University of Liverpool Faculty of Veterinary Science, Liverpool, UK

\section{Introduction/Purpose:}

Developmental stenosis of the cranial thoracic spinal canal has been suggested to occur in large breed dogs, and is documented in the Dogue de Bordeaux (DdB). The purposes of the study were to investigate developmental cranial thoracic stenosis (CTS) further by of the study were to investigate developmental cranial thoracic stenosis (CTS) further by
calculation of reference data in large breed dogs and to determine whether morphometric calculation of reference data in large breed dogs and to deteri
and/or subjective criteria could be used to characterize CTS.

\section{Materials and Methods:}

Magnetic resonance imaging (MRI) studies of large breed dogs (>20 kg), which included transverse images of the first to fifth thoracic vertebrae, were evaluated for the period 2001 2011. A single, blinded observer graded the morphological appearance of the vertebra canal and spinal cord as grade 0 , controls (no bony stenosis), grade 1 , moderately stenotic (stenosis without cord compression), or grade 2, severely stenotic (stenosis with cord compression). Morphometric analysis consisted of cross-sectional area measurements of the vertebral canal at mid-vertebral and intervertebral disc levels to form the canal-area ratio (CAR) and of the spinal cord and vertebral canal at the intervertebral disc level to form the cord-canal ratio (CCR). Normal reference ranges were calculated for CAR and CCR and agreement between the subjective grading and objective data was evaluated. The signalment, neurological presentation, and morphological features of CTS were documented. ment, ne

Seventy-seven MRI studies of the cranial thoracic spine were included. Fifty-eight were graded as controls (grade 0 ) and 19 as moderately or severely stenotic (grade 1 or 2 , respectively). There was a significant difference in CAR and CCR values at different thoracic vertebral sites and between breeds in the control group. CAR discriminated better between the control and stenosis cases than CCR. CCR had poor agreement even with the use of breed specific reference ranges whereas CAR had substantial agreement when breed specific reference ranges were utilized. CTS was recognized in predominantly young (most $<3$ years of age) dogs. Breeds affected included the DdB, Neapolitan mastiff, Bullmastiff, Chow Chow, St Bernard, Staffordshire Bull terrier, German Shepherd dog, Rottweiler, and Doberman. Vertebral morphological changes included enlarged pedicles, facet hypertrophy, altered vertebral body shape, and dorsal lamina cleft formation. In 10/19 of the dogs, CTS was the only spinal abnormality. Eight of these dogs had pelvic limb ataxia and postura reaction deficits and two were neurologically normal. Nine of nineteen cases had concurrent neurological disease of which cervical stenotic myelopathy was the most common. neurological disease of which

Discussion/Conclusion:
Within the control group there was significant variation between breeds and between sites. deally, breed-specific reference ranges should be generated for the ratios at each vertebral site. CAR gave a better discrimination between the control and stenosis cases and had better agreement with the subjective grading system than CCR. CTS should a differential diagnosis in young large breed dogs that present with pelvic limb neurological dysfunction. CTS may be subclinical and should be considered in dogs with cervical stenotic myelopathy.

\section{USEFULNESS OF A HALF-FOURIER ACQUISITION SINGLE-SHOT TURBO SPIN-ECHO PULSE SEQUENCE IN IDENTIFYING ARACHNOID DIVERTICULA IN DOGS}

G.S. Seiler ${ }^{1}$, I.D. Robertson ${ }^{1}$, W. $\mathrm{Mai}^{2}$, W.R. Widmer ${ }^{3}$, J. Suran ${ }^{2}$, S. Nemanic ${ }^{4}$, C.R Lamb $^{5}$, J. Lang ${ }^{6}$, J.L. Johnson ${ }^{7}$, D.E. Thrall ${ }^{1} .{ }^{1}$ North Carolina State University, Raleigh, NC, ${ }^{2}$ University of Pennsylvania, Philadelphia, PA, ${ }^{3}$ Purdue University, West Lafayette, IN, ${ }^{4}$ Michigan State University, East Lansing, MI, ${ }^{5}$ The Royal Veterinary College, University of London, UK, ${ }^{6}$ University of Bern, Bern, Switzerland, ${ }^{7}$ Duke Comprehensive Cancer Center, Durham, NC

\section{Introduction/Purpose:}

Single-shot turbo spin-echo sequences are heavily T2-weighted sequences that are exceptionally well suited to evaluate the subarachnoid space. In the T2-weighted fast spin-echo sequences that are used routinely in spinal magnetic resonance (MR) imaging, the subarachnoid space is not well differentiated from the surrounding epidural fat, which could lead to decreased detection of lesions of the subarachnoid space such as arachnoid diverticula. Our purpose was to determine the added value of a single-shot turbo spin-echo sequence in identifying focal dilations of the subarachnoid space in dogs.
Methods:

MR images of six dogs with a confirmed arachnoid diverticulum, and 24 dogs with other MR images of six dogs with a confirmed arachnoid diverticulum, and 24 dogs with other confirmed spinal disease were included. Six observers were asked to interpret only T2-
weighted images initially, and in a second session T2-weighted and half-Fourier acquisition single-shot turbo spin-echo (HASTE) sequences. The MR images were anonymized, and no signalment, history or clinical information was provided. Each time, the observers were asked to review the images, localize the lesion(s) they considered significant, and to make a diagnosis to the best of their ability. Sensitivity, specificity, positive, and negative predictive values for detection of arachnoid diverticula were calculated and the Wilcoxon signed-rank test was used to determine if the difference in paired observations was statistically significant. Results:

Without the HASTE sequences, $25 \%$ of arachnoid diverticula were identified. Adding the HASTE sequence increased the diagnosis of arachnoid diverticulum to $52.8 \%$. The resulting improvement of $27.8 \%$ after adding the HASTE sequence was statistically significant $(P=0.002)$. No false positive diagnoses of arachnoid diverticulum were made with either sequence. This results in a specificity and positive predictive value of $100 \%$ for both sequences. Mean sensitivity and negative predictive value of the T2-weighted sequence alone were $25 \%$ (range $0-83 \%$ ) and $84.2 \%$ ( $80-96 \%$ ), respectively, and increased to $52.8 \%$ $(0-83 \%)$ and $89.4 \%(80-96 \%)$, respectively, when the HASTE sequence was added.

Discussion/Conclusions:

Although sensitivity in this study was artificially low due to the lack of clinical information provided and variable experience of the observers with the HASTE sequence, the significantly increased detection rate of arachnoid diverticula when using HASTE imaging indicates that this sequence is a valuable addition to MR imaging protocols for the canine spine. Other advantages of this sequence for spinal MR imaging is the speed of acquisition, ability to display changes in CSF composition, and sensitivity to detect extraspinal lesions due to high sensitivity to strongly $\mathrm{T} 2$ hyperintense lesions. Where available, single shot fast spin-echo sequences should be included routinely into spinal MRI protocols.

\section{NOVEL STIR FINDING OF CERVICAL MUSCLE HYPERINTENSITY WITH SUSPECTED} INFLAMMATORY SPINAL CORD DISEASES

S. Eminaga, G.B. Cherubini, E. Villiers, A. Caine. Dick White Referrals, Six Mile Bottom, UK

\section{Introduction/Purpose:}

MRI features of dogs with cervical spinal hyperesthesia and neurological signs suggestive of inflammatory spinal cord diseases are poorly described. MRI signal change affecting muscle has not been noted as a feature of inflammatory spinal cord disease. This retrospective study aimed to assess the sensitivity of a distinctive pattern of STIR muscle hyperintensity for predicting a clinical diagnosis of inflammatory spinal cord disease.

Methods:

All dogs presenting with clinical and neurological signs suspicious of cervical spinal cord disease, which had cervical spinal MRI including STIR sequence and CSF analysis performed between July 2009 and February 2011 were included. Twenty-seven cases fulfilled the inclusion criteria. A board-certified radiologist and neurologist retrospectively reviewed all MRI images to identify any STIR abnormalities and a board-certified clinical pathologist and neurologist reviewed the CSF analysis.

Results:

Thirteen of 27 cases had a distinctive STIR appearance characterized by hyperintensity along the cervical vertebral body extending into the adjacent muscle with an ill-defined margin In $9 / 13$ cases faint to moderate gadolinium-based contrast enhancement was seen margin. In $9 / 13$ cases faint to moderate gadolinium-based contrast enhancement was seen on T1W in the location of the STIR abnormality. Thirteen of the 27 cases had inflammatory had normal STIR images. Two cases with the distinctive STIR changes had normal CSF. The latter two cases were successfully treated for suspected meningoencephalomyelitis of unknown etiology (MUA), so an inflammatory cause was retrospectively diagnosed. In all 13 cases with distinctive STIR changes, the neurological diagnosis was MUA. These results gave the distinctive STIR changes $100 \%$ positive predictive value, $71.4 \%$ negative predictive value, $76.4 \%$ sensitivity, and $100 \%$ specificity for clinical diagnosis of MUA. Discussion/Conclusions:

This is a preliminary report of a novel MRI finding of STIR muscle change, which has been associated with inflammatory cervical spinal cord disease in our small population of dogs. We speculate that the pathogenesis may include extension of the central inflammation into a polyradiculoneuritis, and myositis since the change extends into the adjacent muscles. The ability of STIR to identify the region of interest prior to contrast administration was essential to acquiring images of the affected areas. We propose that this MRI feature may be helpful in the diagnosis of spinal inflammatory disease and STIR sequences should be considered part of the MRI protocol in case of suspected spinal cord inflammatory diseases. 


\section{CONTRAST-ENHANCED CONE BEAM CT IMAGING FOR TUMOR LOCALIZATION} DURING RADIATION THERAPY

$\underline{\text { A. Søvik }}^{1,2}$, J. Rødal ${ }^{2,3}$, H.K. Skogmo ${ }^{1}$, E. Malinen ${ }^{2} .{ }^{1}$ Norwegian School of Veterinary Medicine, ${ }^{2}$ Oslo University Hospital, Oslo, Norway, ${ }^{3}$ The Norwegian University of Science and Technology, Trondheim, Norway

Introduction/Purpose:

Daily cone beam CT (СВCT) imaging is increasingly being used to improve setup accuracy in radiotherapy. СВCT gives images of the patient in the treatment position. These $\mathrm{CBC}$ images are compared to the planning CT scan, and patient position is usually adjusted to achieve the optimal match between bony structures the two image sets, as tumor identification in the CBCT images may not always be possible. The purpose of the present study was to investigate the potential of contrast-enhanced СВCT (СЕСВCT) for tumor localization and treatment monitoring during radiation therapy. and treatmen

Three dogs with spontaneous head and neck tumors were enrolled in a prospective study investigating the role of multimodality tumor imaging for radiation treatment planning an monitoring. The dogs were treated with fractionated IMRT with $6 \mathrm{MV}$ photons to a tota dose of $40-47$ Gy delivered in 10 fractions. Dynamic ${ }^{18}$ F-FDG PET and contrast-enhanced CT (CECT) imaging was performed prior to treatment. CECBCT imaging was performed at 5 of 10 treatment fractions. Subtraction images of postminus precontrast CBCT images were generated to aid in tumor visualization. Furthermore, the CECBCT images were compared with corresponding CECT and PET images to verify the correspondence between the enhancing region in the СBCT images and the tumor volume.

Results:

While unprocessed CECBCT images were judged inadequate for accurate tumor delineation, the tumor volumes were clearly visualized in the CECBCT subtraction images for all dogs at all treatment fractions. Furthermore, the tumor volumes generated from the CECBCT subtraction images showed high spatial correlations with the corresponding CECT and PET images (see figure). For one of the tumors, an adenocarcinoma, a substantial reduction in tumor volume was observed in the CECBCT subtraction images during radiotherapy.

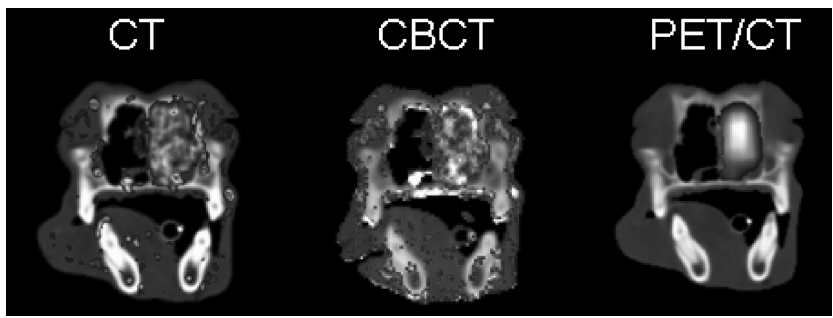

Discussion/Conclusion:

CECBCT subtraction images can be used for tumor localization and monitoring during radiation therapy. Potential applications currently being investigated include automatic corrections of patient positioning based on localization of the tumor volume and adaptation of therapy in response to changes in tumor volumes during treatment. Furthermore, the use of subtraction images to improve visualization of pathological processes, for example, in contrast-enhanced CT and MRI, is being explored.

\section{COMPUTED TOMOGRAPHIC LYMPHOGRAPHY IN THE DIAGNOSIS OF SENTINEL} LYMPH NODE METASTASES IN CANINE MAMMARY GLAND TUMORS: PRELIMINARY RESULTS

C.V. Soultani ${ }^{1}$, M.N. Patsikas ${ }^{1}$, L.G. Papazoglou ${ }^{1}$, N.G. Papaioannou ${ }^{1}$, M Karayiannopoulou $^{1}$, A. Charitanti ${ }^{2}$, P.L. Papadopoulou ${ }^{1}$, E. Flouraki ${ }^{1}$ K. Pavlidou ${ }^{1}$. ${ }^{1}$ School of Veterinary Medicine, ${ }^{2}$ School of Medicine, Aristotle University of Thessaloniki, Greece

\section{Introduction:}

Computed tomographic lymphography (CT-LG) is used in the localization and diagnosis of sentinel lymph node (SLN) metastases of breast cancer in women. It has also been used successfully in the localization of the mammary glands SLNs in normal bitches, but there are no published studies investigating the role of CT-LG in the diagnosis of SLN metastases in canine mammary gland tumors.

\section{Methods:}

Fifteen bitches with unilateral tumors of the third, fourth, and fifth mammary gland were used in this study, since lymph from those glands usually drains into the superficial inguinal lymph nodes, which are easily resected. The SLN of each neoplastic gland was visualized with CT-LG after intratissue injection of $0.5-1 \mathrm{ml}$ of iopamidol $(300 \mathrm{mgl} / \mathrm{ml})$, depending on the size, around the nipple. Transverse images with slice thickness and interslice spacing of $3 \mathrm{~mm}$ were taken from the thoracic inlet to the sacral bone before and within 1 and $5 \mathrm{~min}$ after the injection. In some cases, additional thinly collimated CT images of the SLNs were taken. The same procedure was performed in the contralateral normal gland, which served as normal control. In each injected gland, the topography and pattern of opacification of each SLN were noted. Mastectomy of the neoplastic mammary gland and resection of the SLNs of both sides were performed 2-3 days after CT-LG. The CT-LG findings were correlated with histologic findings.

Results:

The location of each resected node appeared to be consistent with that of CT-LG images. All SLNs of the 15 normal mammary glands opacified fully and homogeneously and were found normal in histologic examination (true negative). Four of 15 SLNs of the neoplastic mammary glands were inhomogeneously opacified and were found to be positive for metastasis in histologic examination (true positive). One of them did not opacify at all, while the others opacified only peripherally or demonstrated irregular opaque spots throughout parenchyma. Ten of 15 SLNs of the neoplastic glands were opacified fully and uniformly and were found free of metastasis (true negative). In one neoplastic gland, the SLN was opacified peripherally only, but was found reactive and not metastatic in histologic examination (false positive).

Conclusion:

Irregular patterns or complete absence of opacification of the SLN in CT-LG may be suggestive of its metastatic status, providing information for tumor staging, treatment and prognosis.

\section{COMPARISON OF SONOGRAPHIC FEATURES OF BENIGN AND NEOPLASTIC DEEP LYMPH NODES IN DOGS}

M. de Swarte ${ }^{1}$, K. Alexander, M.A. d'Anjou, B. Rannou, G. Beauchamp ${ }^{2}$. ${ }^{1}$ University Veterinary Hospital, University College Dublin, Dublin, Ireland, ${ }^{2}$ Département de sciences cliniques, Faculté de médecine vétérinaire, Université de Montréal, St-Hyacinthe, Québec, Canada

Introduction/Purpose

The differentiation of benign versus neoplastic lymph nodes impacts patient management. Specific sonographic features such as size, shape, echogenicity, and Doppler flow pattern are typically considered when assessing lymph nodes in dogs. However, the usefulness of these criteria in distinguishing benign versus malignant lymph nodes remains largely unknown, especially for deep lymph nodes. Our aim was to compare sonographic features in benign and neoplastic deep lymph nodes in order to identify predictive criteria. in benign and

Methods: one cranial mediastinal) in 31 dogs were examined prospectively with B-mode and color flow Doppler. Lymph nodes were aspirated using ultrasound guidance and final diagnoses were established based on cytologic and/or histopathologic interpretation. Lymph nodes were categorized into benign (B: hyperplastic and lymphadenitis) or neoplastic ( $\mathrm{N}$ : Iymphoma, histiocytic sarcoma, and metastasis) groups. Prevalence of each sonographic feature and histiocytic sarcoma, and metastasis) groups. Prevalence of each sonographic feature and
combinations of two features were calculated for each group and compared using a chisquare test or Student's $t$-test for unequal variances. Prevalence of short- and long-axis diameters and short-axis diameter to long-axis diameter (SA/LA) ratio of mesenteric LN were also calculated for each group and compared using a chi-square test.

Results:

Ten lymph nodes were benign (hyperplastic and/or inflammatory) and 21 were neoplastic. All were hypoechoic, except for one neoplastic lymph node. Maximal short-axis diameter $(P=0.0006)$ and long-axis diameter $(P=0.01)$, and SA/LA ratio $(P=0.008)$ were in creased significantly for neoplastic (mean $2.8 \pm 1.7 \mathrm{~cm}, 5.5 \pm 2.4 \mathrm{~cm}$, and $0.50 \pm 0.17 \mathrm{~cm}$ respectively) versus benign (mean $1.2 \pm 0.4 \mathrm{~cm}, 3.8 \pm 1.0 \mathrm{~cm}$, and $0.34 \pm 0.13 \mathrm{~cm}$, respectively) lymph nodes. Specifically, when mesenteric LN measurements were compared, significant difference remained between maximal short-axis $(P=0.01)$ and long-axis diameter $(P=.03)$ but not in SA/LA ratio $(P=0.22)$. The prevalence of other features was similar between groups. Doppler evaluation was possible in $77 \%$ of lymph nodes, with no similar between groups. Doppler evaluation was possible in $77 \%$ of lymph nodes, with no the only significant difference was for the combination of contour regularity and appearance of the perinodal fat $(P=0.03)$

Discussion/Conclusion:

Most ultrasonographic features were not significantly associated with a specific LN category. The greater diameter of neoplastic lymph nodes was consistent with previous studies. Doppler examination of lymph nodes appeared to be of limited clinical value. Despite other reports of the usefulness of combined LN features, only one combination (contour regularity and appearance of the perinodal fat) helped to differentiate benign and neoplastic LN. The small sample size, somewhat different patient size between groups and anatomic variability of the LN herein were study limitations. 


\section{IMAGE-GUIDED, PERCUTANEOUS MICROWAVE ABLATION OF ABDOMINAL AND THORACIC LESIONS IN SMALL ANIMALS}

G.M. Gerboni ${ }^{1}$, F. Rossi ${ }^{2}$, M. Brusati ${ }^{1}$, G. Capra ${ }^{1}$, M. Olivieri ${ }^{1}$, R. Santilli ${ }^{1}$, G.P. Carrafiello ${ }^{3}$ ${ }^{1}$ Clinica Veterinaria Malpensa, Samarate (VA), Italy, ${ }^{2}$ Centro Oncologico Veterinario ${ }^{2}$ Clinica Veterinaria dell'Orologio, Sasso Marconi (BO), Italy, ${ }^{3}$ Dipartimento di Radiologia, Università dell'Insubria, Varese, Italy

\section{Introduction:}

Microwave ablation (MA) is a modern thermoablative technique that enables direct application of heat with consequent cellular death via coagulation necrosis. Under imaging guidance, a microwave antenna is placed directly into the lesion and electromagnetic waves are emitted from the noninsulated part of the antenna. Advantages of MA compared to other thermoablative techniques such as radiofrequency are higher intralesion temperatures, larger ablation volumes, faster ablation times, ability to use multiple applicators, improved convection profile, optimal heating of cystic masses, and less procedural pain. Indications in human medicine include abdominal neoplasia (liver, kidney, adrenals), lung and bone tumors and cystic lesions. Computed tomography (CT) and ultrasound (US) are used to guide the procedure. Contrast-enhanced CT (CECT) and US (CEUS) allow evaluation of vascularization before and after the treatment. Up to date, only experimental studies in research animals are published.

Methods:

Six dogs, four cats, and one ferret with abdominal $(n=6)$, thoracic $(n=4)$ or neck $(n=$ 1) diseases were treated with MA. Lesions included benign hepatic cysts $(n=2)$, adrenal neoplasia $(n=2)$, renal neoplasia $(n=2)$, lung neoplasia $(n=2)$, heart base tumo $(n=2)$, and thyroid adenoma $(n=1)$. All patients had clinical symptoms related to the lesion and they were nonsurgical candidates. Diagnosis was made by US $(n=10)$ or CT $(n=1)$, a contrast-enhanced study was performed and a biopsy was taken. A microwave ablation system (Vivant Medical, Mountain View, CA) was used for the treatment. Under general anesthesia, one or multiple microwave applicators were introduced so that the lesion's volume was covered by the heat-exposed part of the antenna. The applicator was connected to a generator producing $60 \mathrm{~W}$ of power at a frequency of $915 \mathrm{MHz}$. Heating time was between 7 and $10 \mathrm{~min}$ and was followed by a second contrast study. After the procedure, CEUS or CECT were repeated to evaluate residual vascularization.

Results:

MA was performed in all patients without complications. Posttreatment contrast studies showed a lack of enhancement of the lesion in 10/11 cases, only in one large lung carcinoma there was peripheral residual perfusion. Animals recovered quickly without demonstrating pain and could be discharged the day after the procedure. Follow-up imaging showed unchanged volume for the solid lesions in 10/11 cases and almost complete resolution of the cysts. Follow-up varied between 2 weeks and 30 months (mean 7 months). Five animals are still alive, six died or were euthanized because of metastatic diseases or deterioration of the primary conditions. of the primary

Conclusions:

MA is a technically easy and safe technique for treatment of abdominal and thoracic lesions in small animals. MA successfully induced tissue necrosis of solid tumors and resolution of cystic lesions, with a clinically positive result. Validation of these preliminary data with larger number of cases and longer follow-up is necessary. In most cases, MA represents a palliative treatment, therefore careful patient selection is mandatory.

\section{MAXIMUM STANDARD UPTAKE VALUES OF ${ }^{18}$ FDG IN CONFIRMED CANINE PRIMARY MALIGNANT TUMORS AND THEIR METASTASES}

S.L. Kraft ${ }^{1}$, D.S. Gibbons ${ }^{3}$, S. Ryan ${ }^{2}$, S. Lana ${ }^{2}$, J. Custis ${ }^{1}$, S. LaRue ${ }^{1} .{ }^{1}$ Department of Environmental and Radiological Health Sciences, ${ }^{2}$ Animal Cancer Center, Colorado State University, Fort Collins, CO 80523, ${ }^{3}$ Antech Diagnostics, Irvine, CA 92614,

Introduction/Purpose:

The maximum standard uptake value (mSUV) is the most clinically utilized parameter of ${ }^{18} \mathrm{FDG}$ accumulation in tissues, allowing for a semiquantitative estimation of the rate of glycolysis based on radiopharmaceutical metabolism. ${ }^{1}$ The pattern of ${ }^{18} \mathrm{FDG}$ uptake and standard uptake values have been described in the normal $\operatorname{dog}^{1}$ and $\mathrm{cat}^{2}$, and the pattern of ${ }^{18} \mathrm{FDG}$ uptake was described in dogs diagnosed with lymphoma and mast cell tumors. ${ }^{3}$ This report presents the mSUVs of confirmed canine primary malignant tumors and/or their

metastases.

Methods:
${ }^{18}$ FDG-PET/CT imaging was performed on 11 dogs diagnosed with a primary malignant tumor, and one dog for metastasis of a known primary malignant tumor. One dog underwent second ${ }^{18}$ FDG-PET/CT procedure following stereotactic body radiation therapy of a primary bone tumor. Whole body CT and PET imaging was performed using a standard protocol, with
PET images acquired $60 \mathrm{~min}$ following ${ }^{18} \mathrm{FDG}$ administration for all dogs. Tumor volume and mSUVs were derived from commercial software (Extended Brilliance Workstation, Phillips Medical Systems Philips Healthcare, 3000 Minuteman Road, Andover, MA).

Results:

Eight breeds were represented. Ages ranged from 4.98 to 12.92 years, (mean 9.44 years) Twelve primary tumor types were identified in 11 dogs. Metastasis was identified in three of these 11 dogs and in the dog imaged specifically to determine the extent of metastatic disease secondary to left humeral osteosarcoma. Thirteen metastatic lesions (regional lymph nodes, lung, liver, and pleura/mediastinum) were identified. Primary tumor volumes ranged from 1.46 to $160.6 \mathrm{~cm}^{3}$, with a mean of $57.96 \mathrm{~cm}^{3}$. Maximum SUVs of the primary tumors ranged from 3.4 to 25.9 , with a mean of $11.72(n=12)$. Maximum SUVs for metastatic lesions ranged from 1.9 to 7.8 , with a mean of $4.45(n=13)$ and mean volume of $30.98 \mathrm{~cm}^{3}$ (range $0.48-254.86 \mathrm{~cm}^{3}$ ).

Discussion/Conclusions:

A wide range of tumor volumes and $\mathrm{mSUVs}$ were detected for canine primary and metastatic neoplasms. Primary tumor mSUV was not related to its tumor volume $\left(R^{2}=0.0983\right)$ However, the lower mSUVs of metastatic lesions may relate, at least in part, to some of the lesion volumes being small relative to PET spatial resolution. Maximal SUVs have prognostic value for many human malignancies ${ }^{1}$ but a similar utility of canine mSUVs has not yet been determined as this technology is new to veterinary medicine. Maximal SUVs are influenced by numerous factors including tumor volume, histology, anatomic location, plus equipment, and protocol factors. ${ }^{1}$

References:

1. LeBlanc, AK, Jakoby, B, Townsend, DW, Daniel, GB. Vet Rad \& Ultrasound 2008; 49(2):182-188.

2. LeBlanc, AK, Wall, JS, Morandi, F, et al. Vet Rad \& Ultrasound 2009; 50(4):436-441.

3. LeBlanc, AK, Jakoby, BW, Townsend, DW, Daniel, GB. Vet Rad \& Ultrasound 2009; 50(2):215-233.

\section{ONLINE DOSIMETRY FOR TEMOPORFIN-MEDIATED INTERSTITIAL PHOTODYNAMIC THERAPY OF THE PROSTATE IN DOGS}

J. Swartling ${ }^{1}, \underline{\text { K. Hansson }}{ }^{2}$, O. Höglund ${ }^{2}$, F. Södersten ${ }^{3}$, J. Axelsson ${ }^{1}$, A-S. Lagerstedt ${ }^{2}$ ${ }^{1}$ Spectracure AB, Lund, Sweden, ${ }^{2}$ Department of Clinical Sciences, ${ }^{3}$ Department of Biomedical Sciences and Veterinary Public Health, SLU, Uppsala, Sweden

Introduction:

Prostate cancer is the most common cancer in men. Interstitial photodynamic therapy (PDT) is an alternative treatment. The dog prostate model has been used in previous studies of interstitial PDT but with no or only limited PDT dosimetry. The purpose of the present study was to verify the efficacy of online dosimetry as used by the SpectraCure P18 system using the dog prostate as model with temoporfin as photosensitizer. The study was designed to address two issues: (1) the correlation between dose plans and tissue response evaluated by magnetic resonance imaging (MRI) and histopathology, (2) the light dose needed to achieve necrosis of prostate tissue.

\section{Methods:}

Nine clinically healthy intact male Beagles divided into three groups were used. Temoporfin (Foscan ${ }^{\circledR}$, Biolitec Pharma, Germany) was administered 3 days before PDT. Transrectal ultrasound was used to create a pretreatment dose plan. Seven 18-guage needles were inserted transperineally under ultrasound guidance, aided by a mechanical template locked to the ultrasound probe. Optical fibers were inserted into the needles for light delivery and dosimetry. A fixed light dose was used for the initial group of three dogs and online dosimetry for the following two groups. MRI was performed before administration of temoporfin and on day 7 after PDT treatment in a 0.27-T permanent magnet (Hallmarq Veterinary Imaging) using T1W SE precontrast and postcontrast and T2W FSE sequences. Directly after the second MRI examination, the dogs were euthanized and subjected to autopsy.

Results:

All dogs showed similar intensity changes on MRI with a marked contrast enhancement in T1W SE and slight to moderate hyperintensity in T2W FSE that corresponded to hemorrhagic changes on histopathology. Areas of signal void in contrast-enhanced T1W SE scans were seen in all but two dogs. These were found to be necrotic foci. In all dogs, the prostatic capsule was irregular and diffuse and the periprostatic fatty tissue contained irregular linear structures. Intensity changes in the ventral rectum were seen in four dogs. Dose planning and online dosimetry led to improved treatment results, both in terms of focusing the PDT effect to the target volume and to achieve the objective of attaining the threshold dose to induce necrosis. A light dose-response relationship was established where the threshold dose to induce prostate gland necrosis was estimated to $20-30 \mathrm{~J} / \mathrm{cm}^{2}$.

Conclusion:

The results show that temoporfin-mediated PDT can cause necrosis of the canine prostate. PDT is a potential treatment alternative for prostate cancer. 


\section{THE VALUE OF RADIOGRAPHY FOR EVALUATION OF THE MORPHOLOGY OF DISTAL BORDER SYNOVIAL INVAGINATIONS OF THE DISTAL SESAMOID BONE IN
THE HORSE}

S. Claerhoudt ${ }^{1}$, H.J. Bergman ${ }^{2}$, H. van der Veen ${ }^{2}$, L. Duchateau ${ }^{3}$, K. Vanderperren ${ }^{1}$ E.V. Raes ${ }^{1}$, J.H. Saunders ${ }^{1} .{ }^{1}$ Veterinary Medical Imaging, Faculty of Veterinary Medicine, Ghent University, Merelbeke, Belgium, ${ }^{2}$ Lingehoeve Diergeneeskunde/VetCT, Lienden, The Netherlands, ${ }^{3}$ Department of Physiology and Biometry, Faculty of Veterinary Medicine Ghent University, Merelbeke, Belgium

\section{Purpose:}

To investigate the value of radiography in the evaluation of the morphology of distal border synovial invaginations in the horse.

Methods:

Computed tomography (CT) scans and three dorsoproximal-palmarodistal oblique (DPr$\mathrm{PaDiO}$ ) radiographs were obtained on 50 cadaver forefeet from 25 Warmblood horses. C was assumed to be the gold standard. The number, shape, and depth of penetration of distal border synovial invaginations into the distal sesamoid bone (DSB) were evaluated with both methods, and the comparison of their measurements was statistically described.

Results:

A statistically significant mean difference for number of distal synovial invaginations between $\mathrm{CT}$ and all three $\mathrm{DPr}-\mathrm{PaDiO}$ projections was found, and approximately equal to 2 , meaning that CT permits visualization of an average of 2 more invaginations compared to radiography. In none of the cases, radiography had a higher number observed than $\mathrm{CT}$. A large variation in the difference of measurements for depth of penetration against their mean difference between $\mathrm{CT}$ and the three radiographic projections was seen. Radiography was underestimated when high mean values applied. There was no statistically significan mean difference found between both techniques for depth. A moderate to good agreement between measurements on CT and the three DPr-PaDiO projections for shape was seen, in which the D55 $\mathrm{Pr}-\mathrm{PaDiO}$ projection showed the best agreement. A high specificity (90-99\%) and low sensitivity $(65 \%)$ for all projections for shape were found.

Discussion and Conclusion:

For the evaluation of the number, depth, and shape of distal synovial invaginations in the $\mathrm{DSB}$, radiography shows only partially the morphology seen on CT and is thus of very limited value for evaluation of the distal navicular border synovial invaginations.

\section{OSSEOUS CYST-LIKE LESIONS IN THE NAVICULAR BONE OF LAME HORSES}

M. Biggi, S. Dyson. Centre for Equine Studies, Animal Health Trust, Lanwades Park, Kentford, CB8 7UU, UK

\section{Introduction/Purpose:}

Osseous cyst-like lesions (OCLLs) are occasionally observed on magnetic resonance (MR) images as fluid-filled lesions in the distal third of the navicular bone, but their clinical significance is unclear. We aimed to evaluate the distribution of OCLLs in lame horses and to evaluate their potential contribution to lameness by analyzing MR images of lame horses.

Methods:

Horses had forelimb or hindlimb lameness abolished by palmar/plantar digital nerve blocks performed at the base of the proximal sesamoid bones. Horses were included if an OCLL was observed on MR images, defined as a discrete fluid-filled lesion surrounded by a mineralized rim extending from the palmar/plantar to the dorsal cortex of the navicula bone, separate from synovial invaginations. The lateromedial distribution of OCLLs was recorded; additional lesions in the navicular bone were also documented. Radiographs were evaluated, if available, to determine sensitivity and specificity of radiology for detection of OCLLs identified on MR images. Final diagnosis was determined both prospectively of OCLLs identified on MR images. Final diagnosis was determined both prospectively
based on responses to diagnostic analgesia and the result of all imaging techniques and retrospectively by evaluation of MR images for the presence and extent of lesions involving all structures in the digit. Lesions were classified as primary deep digital flexor tendon injury, navicular bone lesion with or without desmitis of the distal sesamoidean impar ligament (DSIL) or collateral sesamoidean ligaments (CSL) and "other" lesions.

Results:

Thirty-nine horses were included and 55 OCLLs were observed in 77 feet. There was no difference in the lateromedial distribution of the OCLLs, however lesions were observed more commonly at the junction between the distal horizontal and sloping borders then a other locations. A total of $58.2 \%$ of the OCLLs were seen proximal to a distal border fragment; an additional $14.5 \%$ of the navicular bones had a fragment, but the OCLL was not closely related to it A total of $84.2 \%$ of the unilaterally lame horses had the OCLL in the lame limb; $76.0 \%$ of the bilaterally lame horses had an OCLL in the lamest limb. However, in $15.8 \%$ of unilaterally lame horses an OCLL was observed in the nonlame limb only. Radiographs were available for 33 horses; the majority of the OCLLs were observed on radiographs as a discrete or ill-defined radiolucency immediately proximal to the distal horizontal or sloping border. Sensitivity and specificity of radiology for detecting OCLLs using MR imaging as the gold standard were 93.1 and 76.9 , respectively. In all horses navicular disease, with or without desmitis of the DSIL or CSL, was considered likely to contribute to pain and lameness; in $41 \%$ of horses, navicular disease was the only diagnosis. In $20.5 \%$ of horses a DDFT lesion was considered to be a major contributor to lameness. The remaining horses had "other" lesion(s) in addition to navicular bone disease, which may have contributed to lameness.

Discussions/Conclusions:

OCLLs are observed most commonly in a navicular bone with distal border fragments and are often located proximal to a fragment. Navicular bone disease characterized by $\mathrm{OCL}$ in the distal third of the bone may be the only source of pain in some horses. A careful evaluation of radiographs allows identification of the majority of OCLLs.

\section{COMPARISON OF RADIOGRAPHY AND MRI FOR THE EVALUATION OF NAVICULAR} BONE ABNORMALITIES IN HORSES

B. Marquis, K. Alexander, Y. Rossier, G. Beauchamp. Faculté de médecine vétérinaire Université de Montréal, St-Hyacinthe, Québec, Canada

\section{Introduction:}

"Navicular syndrome" is a series of diseases affecting the soft tissue and osseous structures of the palmar pedal region in horses. Radiographs are traditionally used for initial screening due to their low cost and broad availability, yet MRI is superior for evaluating soft tissues and certain osseous lesions such as bone marrow edema. Nonetheless, few guidelines exist for evaluating navicular bone abnormalities during MRI of the distal limb. We hypothesized that certain features of navicular bone disease could be evaluated by adapting traditional radiographic criteria to $\mathrm{MRI}$.

\section{Methods:}

Images of horses that received radiographs and MRI examination of the distal limb were retrospectively reviewed randomly and independently by three observers. A detailed interpretation grid was applied that used similar criteria for evaluating various features of navicula bone disease on both MRI and radiographs: enthesophyte formation, distal border fragmentation, medullary sclerosis, synovial invagination modifications, flexor cortex erosions, and cyst-like lesions. Radiographic and MRI findings were compared. Interobserver agreement was also calculated separately for each radiographs and MRI

Results:

Thirty-one feet in 22 horses were examined. Of these, 24 feet had soft tissue or osseous signs causing palmar pedal pain. Between MRI and radiographs, a statistically different prevalence of positive findings was found for medial distal border defects (MRI 29\%, radiographs $7 \%$ of cases), marked synovial invaginations (MRI $23 \%$, radiographs $7 \%$ ), medullary sclerosis (MRI 61\%, radiographs 39\%), and poor corticomedullary definition (MRI 16\%, radiographs $32 \%$ ). Between modalities, there was substantial agreement for lateral distal border fragmentation ( $k 0.76$ ). Moderate agreement was found for corticomedullary definition $(k 0.58)$, flexor cortex thickening $(k 0.51)$, lateral enthesophyte formation $(k 0.46)$, and tion $(k 0.58)$, flexor cortex thickening $(k 0.51)$, lateral enthesophyte formation $(k 0.46)$, and medullary sclerosis $(k 0.45)$. Fair-to-poor agreement was found for synovial invagination
modifications $(k 0.38)$, medial border fragmentation $(k 0.35)$, proximal and medial border enthesophyte formation ( $k 0.22$ and 0.14 , respectively), lateral and medial distal border defects ( $k 0.35$ and $k 0.09$, respectively), and flexor cortex erosions/cysts $(k 0.21)$. Interobserver agreement was generally higher for radiographic parameters $(k$ median 0.47 , range $0-1$ ) than for MRI ( $k$ median 0.32 , range $0-0.78$ ), with the exception of distal border fragmentation, where MRI agreement was higher among all three observers.

where MRI agre

Navicular bone abnormalities with moderate-to-substantial agreement have the potential to be appropriately assessed on MRI using criteria extrapolated from traditional radiographic evaluation. A higher prevalence of some findings on MRI may indicate an improved ability to assess the navicular bone, although this remains to be proven. Greater interobserver variability on MRI should warrant some caution when evaluating navicular bone morphologic abnormalities using this emerging modality and likely reflects the current paucity of specific interpretation guidelines.

\section{STANDING LOW-FIELD MAGNETIC RESONANCE IMAGING (MRI) OF THE FOOT IN 205 HORSES (2007-2009) AND ANALYSIS OF OUTCOME ACHIEVED}

J. Dixon ${ }^{1}$, R. Weller ${ }^{1}$, N. Bolas ${ }^{2}$, T. Mair ${ }^{3} .{ }^{1}$ The Royal Veterinary College, University of London, ${ }^{2}$ Hallmarq Veterinary Imaging Ltd., Guildford, ${ }^{3}$ Bell Equine Veterinary Clinic, Maidstone, UK

Introduction:

In the last decade MRI has opened a new diagnostic chapter in lameness diagnosis, with over 20,000 completed low-field standing MRI scans since 2003. However, little is still un derstood about the consistency of findings from MRI within a population, and long-term outcome. This study evaluated use of standing MRI for equine lameness in a referral population, and reported on its impact upon long-term outcome. Hypotheses: (1) specific lesions detected at MRI significantly correlate with a poor outcome, in particular deep digital flexo tendinopathies; (2) maximal athletic performance reached post-MRI will be significantly worse than prior to lameness.

\section{Methods:}

Retrospective evaluation of cases $(n=205)$ subjected to MRI of the foot for lameness (20072009) was undertaken with information collected including: history, signalment, lameness duration and grade, and prior diagnostics. MRI lesions were categorized by structure and severity. Long-term follow-up assessment was made and outcome assessed with respect to the imaging diagnoses, athletic performance, and lameness duration.

Results:

Successful outcome (sound with a full return to work) was attained in $32.7 \%$ of cases Common MRI findings included DDFT, navicular bone, and collateral ligament of the distal interphalangeal joint lesions. Outcome was significantly worse for cases where concurren DDFT and navicular bone lesions occurred. Lameness duration was not observed to be a factor influencing recovery. Athletic performance post-MRI and recovery was found to be significantly worse than prior to lameness.

Conclusions:

Soft-tissue lesions in the equine distal limb continue to be associated with unsuccessful outcomes. Combined DDFT and navicular bone lesions are associated with a poor prognosis. An enhanced understanding of lesions may allow more targeted treatment and improve outcome. A greater understanding of interlesion relationship can therefore be developed. Use of MRI to detect early/subclinical disease may allow effective preventive strategies to be adopted. 


\section{MAGNETIC RESONANCE IMAGING IN FOALS WITH INFECTIOUS ARTHRITIS}

L. Gaschen, A. LeRoux, J. Trichel, L. Riggs, H.H. Bragulla, N. Rademacher, D. Rodriguez. Department of Veterinary Clinical Sciences, Louisiana State University, Baton Rouge, LA

\section{Introduction/Purpose:}

The purpose of this study is to describe and establish MRI findings in foals with noninfectious and infectious arthritis and to correlate these findings with currently accepted diagnostic tests, that is, radiography and joint fluid analysis. We hypothesize that abnormalities in the tests, that is, radiography and joint fluid analysis. We hypothesize that abnormalities in the better with MRI than radiography.

\section{Methods:}

Client-owned foals younger than 4 months of age that presented with joint effusion, pain and swelling affecting the fetlock, carpus, and/or tarsus were included in the study. Foals were either examined under anesthesia or immediately post euthanasia. Joint fluid analysis, digital radiographic, and MRI examinations were performed in each foal. Foals were categorized into two groups, septic and nonseptic arthritis, based on joint fluid analysis. The following pulse sequences were performed on each affected joint: sagittal T2, STIR, RSSG 3D Water Excitation and PD, transverse PD, and T2 and dorsal T2-weighted pulse sequences.

Results:

A total of 28 joints were examined in six septic, three nonseptic and one control foal ranging in age from 1.5 to 16 weeks. A total of 14 septic joints and seven nonseptic joints were examined in nine foals. Nineteen bone lesions in six septic joints were detected with MRI and only four of these lesions were detected radiographically. T2, STIR, and PD pulse sequences demonstrated 19 hyperintense bone lesions with a hypointense halo in six differen joints in four of the six septic foals, but not in those with nonseptic arthritis or in the one foa with presumed normal joints. All except for one lesion was hyperintense on additional RSSG Water Excitation pulse sequences and the contrast with the signal void of the surrounding bone made them stand out more than on other pulse sequences. Four lesions were epiphyseal, four metaphyseal, five physeal, five in cuboidal bones, and one lesion was detected in seal, four metaphyseal, five physeal, five in cuboidal bones, and one lesion was detected in
articular cartilage. The three foals with nonseptic arthritis showed no bone lesions in MRI articular cartilage. The three foals with nonseptic arthritis showed no bone lesions in MRI
or radiographically. Four of the six foals with septic arthritis had to be euthanized due to or radiographically. Four of the six foals with septic arthritis had to be euthanized due to
the severity of the disease. These foals had a minimum of one to maximal six MRI lesions per joint, whereas two of the surviving septic arthritis foals had no bone lesions and one of these had only two lesions in one joint. Of the six joints that had bone lesions detected with $\mathrm{MRI}$, three had at least one lytic lesion detected radiographically.

Discussion/Conclusion:

MRI appears to be better than radiography in the detection of bone lesions in foals with septic arthritis. Hyperintense T2, STIR, PD, and RSSG Water Excitation subchondral lesions were the most common bony abnormalities in septic joints. Foals with septic arthritis are mor likely to have heterogenous signals in the joint fluid of affected joints compared to those with nonseptic arthritis. Assessment of cartilage damage with MRI warrants further investigation and comparisons with histology. The results of this study warrant further investigation as to the predictive value of the presence of osteomyelitis detected in MRI and the prognosis of the foal. Osteomyelitis may be clinically indistinguishable from septic arthritis and preintervention foal. Osteomyelitis may be clinically indistinguishable f

\section{ULTRASONOGRAPHY OF THE COLLATERAL LIGAMENTS OF THE DISTAL} INTERPHALANGEAL JOINT IN HORSES: TECHNIQUE AND REFERENCE IMAGES

L. Evrard ${ }^{1}$, G. Bolen ${ }^{1}$, N. Maquet ${ }^{2}$, V. Busoni ${ }^{1} .{ }^{1}$ Department of Clinical Sciences of Companion Animals and Equids, Faculty of Veterinary Medicine, Diagnostic Imaging Section, University of Liège, ${ }^{2}$ University of Liège, Faculty of Medicine, Department of Clinical Sciences, Diagnostic Imaging Section, Liège, Belgium

\section{Introduction/Purpose:}

Desmopathies of the collateral ligaments (CLs) of the distal interphalangeal joint (DIPJ) are a common cause of foot lameness in horses. The purposes of this study were to describe ultrasound (US) technique and reference images of the equine DIPJ CLs and to evaluate ultrasound (US) technique and reference images

the CLs portic

Transverse and longitudinal US images were obtained (3500 SSD, Aloka N.V./S.A., Meche len, Belgium - 7.5 MHz linear transducer) on 15 equine cadaver forelimbs and two horse destined to euthanasia for medical reasons unrelated to lameness. All the feet were examined by magnetic resonance imaging (MRI; Trio, Siemens, Marie Curiesquare, Bruxelles, Belgium - knee human radiofrequency coil) to confirm normalcy. On six limbs a needle was placed under US-guidance at the distal limit of US visualization of each CL. The distance between needle and CL proximal insertion fossa in comparison to the distance between proximal and distal insertion fossae was evaluated on computed tomography (CT) images proximal and distal insertion fossae was evaluated on computed tomog
(16 slices Somatom, Siemens, Marie Curiesquare, Bruxelles, Belgium). Results:

All limbs were normal at MRI. Four US transverse images were obtained with the probe placed on the coronary band and using an increasing angle between US beam and horizontal plane. A centro-dorsal hypoechoic image appeared with increasing probe inclination. On the most distal transverse US image, CLs appeared homogeneously hypoechoic. Two fascicles, a deep and a superficial, distally divergent, were visible on longitudinal images. The proportion of CL accessible by US estimated at CT was more than $50 \%$ in $9 / 12$ CLs. Discussion/Conclusions:

Different fiber bundles oriented in different planes are visible on CLs longitudinal images and create a hypoechoic image appearing because of anisotropy with increased probe angulation. Awareness of this morphology is crucial to reduce false positive results on clinical cases. The proportion of the CL accessible by US is higher than what suggested by previous literature.

\section{COMPARATIVE IMAGING OF EQUINE FETLOCK INJURIES: RADIOGRAPHY, ULTRASONOGRAPHY, AND STANDING MRI}

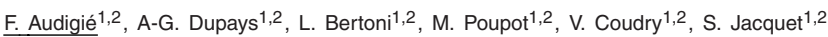
J-M. Denoix ${ }^{1}, 2$, 1 Université Paris-Est, Ecole Nationale Vétérinaire d'Alfort, CIRALE-14430 Goustranville, ${ }^{2}$ INRA, USC BPLC 957-94704 Maisons-Alfort, France

\section{Introduction/Purpose:}

Standing MRI is used to further investigate equine distal limb injuries particularly when conventional imaging modalities are inconclusive or when a more complete evaluation is needed. The purposes of this study were to evaluate the diagnostic performance of standing MR compared to conventional imaging in fetlock disorders and to describe the corresponding abnormal MR findings.

Methods:

A retrospective study was conducted on 40 clinical cases $(20$ sports horses and 20 racing ones) referred at the CIRALE for MR evaluation of the fetlock or distal limb. In all patients, the definitive diagnosis confirmed that fetlock injuries had or might have a clinical significance. Radiographic and ultrasonographic examinations were conducted prior to standing MRI. For all cases, the diagnostic performance (DP) of standing MRI with respect to conventional imaging was quantified on a nine-grade scale where $-4=$ major clinical injuries identified by conventional imaging but undiagnosed by MRI; $0=$ no MR diagnostic improvement; $+4=$ MR identification of major clinical injuries undiagnosed by conventional imaging. Statistica analysis was performed using either $\chi^{2}$ or Student $t$-tests $(P<0.05)$.

Results:

Examinations were mainly performed on the forelimbs $(77.5 \%$ of horses). Bilateral fetlock imaging was acquired in $65 \%$ of patients. Injuries identified were of four main types: bone contusion (presence of increased signal on STIR), bone sclerosis, osteoarthrosis, and ligament injuries. The distribution of these entities was statistically different between both groups with a higher frequency of bone contusion in racing horses ( $80 \%$ of patients) compared to sport horses $(30 \%)$, which showed a higher proportion of osteoarthrosis and ligament lesions. Bone contusion injuries were more frequently dominant in the metacarpal/tarsal condyle $(76 \%)$ followed by the proximal aspect of the first phalanx (18\%) and the proximal sesamoid bones $(6 \%)$. Differences were also found in the pattern of subchondral and cancellous bone alterations between patients and types of injuries. The mean DP of fetlock cellous bone alterations between patients and types of injuries. The mean DP of fetlock
standing MRI was $2.6 \pm 1.9$ with higher values for bone injuries $(3.6 \pm 0.7)$ compared to standing MRI was $2.6 \pm 1.9$ with higher values for bone injuries $(3.6 \pm 0.7)$ compared to
ligament injuries $(1.0 \pm 1.7)$ and osteoarthrosis $(0.1 \pm 2.2)$. Finally, the DP of standing MR was significantly greater for racing horses $(3.5 \pm 1.1)$ compared to sport ones $(1.7 \pm 2.2)$. Discussion/Conclusions:

For osteoarthrosis and ligament injuries, MR examination allows mainly to rule out other potential injuries undiagnosed with conventional imaging. An excellent correlation was found between standing MR and conventional imaging findings for ligament injuries, except for palmar ligament lesions where ultrasonography was more sensitive for identifying enthesophytes and MRI for detecting alterations of the bony component of the enthesis. For osteo-arthrosis, diffuse alterations of the articular cartilage diagnosed with ultrasonography have remained undetected by standing MRI whereas focal cartilaginous lesions have been diagnosed on standing MRI and not on ultrasonography because of their location in nonaccessible part of the joint. In conclusion, standing MRI does not replace conventional imaging but represents an excellent complementary imaging technique for further investigating fetlock conditions. 


\section{RADIOLOGICAL AND SCINTIGRAPHIC EVALUATION OF THE THIRD CARPAL BONE OF HORSES WITH CARPAL LAMENESS AND CONTROL HORSES}

V. Simon ${ }^{1}$, S.J. Dyson ${ }^{2} .{ }^{1}$ The Equine Veterinary Clinic, Houston, Renfrewshire, ${ }^{2}$ Centre for Equine Studies, Animal Health Trust, Newmarket, Suffolk, UK

\section{Introduction/Purpose:}

Increased radiopacity (IRO) of and increased radiopharmaceutical uptake (IRU) in the equine third carpal bone (C3) have been described both as a normal adaptation to training and as a pathological condition. There are limited data on the association between radiological and scintigraphic findings of the $\mathrm{C} 3$ and their association with lameness in sports horses. The aims of this study were (1) to document the radiological and scintigraphic findings of the $\mathrm{C} 3$ of horses performing different work disciplines, (2) to compare the radiological and scintigraphic findings, and (3) to investigate their relationship with lameness.

Methods:

A total of 153 lame or poorly performing horses were included in the study; 83 had carpal radiographs including a dorsoproximal-dorsodistal oblique image of the distal row of carpa bones; 100 had ung dorsoproximal-dorsodistal oblique image of the distal row of carpa work discipline were recorded. The radiographic and scintigraphic images of the lamest limb of each horse were analyzed. The IRO of the C3 was graded. The site and intensity of IRU in the distal row of carpal bones was graded subjectively. The ratio of radiopharmaceutical uptake (RU) between the distal row of carpal bones and the reference region (distal radius) was determined using region of interest analysis. A chi-square test was use (distal radius) was determined using region of interest analysis. A chi-square test was used findings and discipline and lameness groups. Kruskal-Wallis ANOVA was used to test fo associations between the ratio of RU and the discipline and lameness group. A Spearman correlation was used to test for associations between radiological and scintigraphic features.

Results: IRO was found in horses of all disciplines, with racehorses having the highest frequency
$(P=0.009)$. The ratio of RU was associated with the discipline $(P=0.026)$. There was an $(P=0.009)$. The ratio of RU was associated with the discipline $(P=0.026)$. There was an
association between the severity of IRO and both subjective IRU in the C3 $(P=0.002 ; r s=$ $0.59)$ and ratio of $\mathrm{RU}(P=0.013 ;$ rs $=0.46)$. IRO of and subjective IRU in the C3 could be observed independently. Lameness related to the middle carpal joint was associated with IRO $(P<0.001)$, ratio of $\mathrm{RU}(P=0.037)$, and subjective IRU in the distal row of carpal bones $(P<0.001)$. IRO was associated with radiological signs of osteoarthritis of the middle carpal joint $(P=0.003)$, large medullary lucencies $(P<0.001)$, and marginal lucencies in the $\mathrm{C} 3$ joint $(P=0.003)$, large medullary lucencies $(P<0.001)$, and marginal lucencies in the
$(P<0.001)$. Subjective IRU in the distal row of carpal bones was associated with radiological signs of osteoarthritis of the middle carpal joint $(P=0.006)$ and marginal lucencies in the C3 $(P=0.008)$. Horses with medullary vascular channels were underrepresented in horses with subjective IRU in the distal row of carpal bones $(P=0.012)$.

Discussion/Conclusions:

High-speed exercise may not be the only determining factor in the development of osseous pathology of the C3. Although IRO of and IRU in the C3 were associated, they were not always observed concurrently. Radiology performed in conjunction with scintigraphy may improve the detection of horses with osseous pathology of the C3. Both IRO and IRU were more likely to be seen in horses with lameness related to the middle carpal joint, than horse with other sources of pain.

\section{INCOMPLETE LONGITUDINAL FRACTURES AND FATIGUE INJURY OF THE PROXIMOPALMAR MEDIAL ASPECT OF THE THIRD METACARPAL BONE IN 55 HORSES}

R. Morgan $^{\star}$, S. Dyson. Centre for Equine Studies, Animal Health Trust, Lanwades Park Newmarket, "Fellowes Farm Equine Clinic, Abbots Ripton, Cambridgeshire, UK

\section{Introduction/Purpose:}

Previous descriptions of incomplete longitudinal fractures and stress-related bone injury of the proximopalmar aspect of the third metacarpal bone (McIII) have focused on diagnostic imaging findings, especially in racehorses. The objectives were to document the signalment of horses, clinical features, response to diagnostic analgesia, diagnostic imaging findings, and follow-up data in a large group of horses with an incomplete longitudinal fracture or stress-related bone injury of the proximopalmar medial aspect of the Mclll.

stress-related

Horses were included in the study if pain was localized to the proximopalmar aspect of the metacarpal region, with radiological evidence of an incomplete longitudinal fracture or generalized increased radiopacity in the proximopalmar medial aspect of the Mclll, or focal increased radiopharmaceutical uptake (IRU) in the proximopalmar aspect of the Mclll. Age, breed, gender, height, bodyweight, work discipline, work history, duration of lameness, clinical signs, and responses to diagnostic analgesia were recorded. Radiographic and scintigraphic images were assessed subjectively and objectively.

Results:

There were 55 horses representing a broad spectrum of ages (2-14 years), breeds (Thoroughbred, Thoroughbred cross, Warmblood, and others), and work disciplines (eventing, hunting, general purpose, showjumping, dressage, jump racing, flat racing, endurance), $72 \%$ of which had radiological abnormalities. Twelve percent had increased radiopacity alone, $20 \%$ had a radiolucent line consistent with a fracture in the proximomedial aspect of the McIII, and $42 \%$ had a fracture and increased radiopacity. Fractures were orientated either parallel to the long axis of the bone or in a proximomedial to distoaxial direction. The majority had no localizing clinical signs, although $73 \%$ of horses with radiological abnormalities showed a characteristic pattern of lameness. Horses with and without a radiologically evident fracture line had similar lameness grades. Lameness was generally worse in straight lines than in circles. IRU ranged from mild to intense in the lame limb; $14 \%$ of nonlame limbs had mild IRU. Fifty percent of lame limbs had both radiological abnormalities and IRU in the proximopalmar aspect of the McIII, $50 \%$ had only IRU in the proximopalmar aspect of the Mclll. Ninety-eight percent of horses for which long-term follow-up was available returned to full athletic function. Fracture lines reexamined radiologically 3 months later were generally shorter with reduced opacity of the Mclll. Repeat scintigraphy showed RU had decreased by at least one grade in all limbs.

Discussion/Conclusions:

Incomplete longitudinal fractures and stress-related bone injury of the proximopalmar media aspect of the Mclll may occur in horses of many types and sports disciplines and are not confined to immature athletes. They should be considered an important differential diagnosis for proximal metacarpal region pain.

\section{IMAGING FEATURES AND HISTOLOPATHOGIC FINDINGS OF EXOSTOSES OF THE AND ASPECT}

L. Bertoni $^{1,2}$, V. Coudry ${ }^{1,2}$, F. Audigié ${ }^{1,2}$, J-M. Denoix ${ }^{1,2}$. ${ }^{1}$ Université Paris-Est, Ecole Nationale Vétérinaire d'Alfort, CIRALE-14430 Goustranville, ${ }^{2}$ INRA, USC BPLC 957 - 94704 Maisons-Alfort, France

\section{Introduction:}

Exostoses of the palmar and plantar cortex of the third metacarpal (Mc3) and third metatarsa (Mt3) bones located between the middle and the distal third of the bone diaphysis should be considered as a cause of lameness in horses. The purpose of this paper is to describe the radiographic, ultrasonographic, scintigraphic, and magnetic resonance imaging (MRI) features of this specific condition, together with the results of histopathologic analysis.

\section{Methods:}

Between 2001 and 2011, seventeen horses with unilateral or bilateral exostosis of the palmar/plantar cortex of the Mc3/Mt3 bone were diagnosed at the Center of Imaging and Research on the Equine Locomotor Affections (CIRALE). Signalment, case history, results of clinical examination, imaging, surgical, and histopathologic findings were retrospectively evaluated.

Results:

Nine horses had unilateral lameness of the limb affected by the exostosis, abolished by local or perineural analgesia while eight horses showed no consistent lameness at the time of examination. The diagnosis was made with radiography and ultrasonography in all cases. Forelimbs were preferentially involved ( $94 \%$ of cases), and the lesion was bilateral in $35 \%$ o cases. Desmopathy and/or peritendinous fibrosis of the third interosseous muscle (TIOM) a the exostosis site were identified in $35 \%$ of cases. Nuclear scintigraphic bone phase images obtained in four cases revealed no change in radiopharmaceutical uptake (RU) in three cases and a focal increase in RU in one case. Low-field MR examination performed in two cases showed cortical bone proliferation with slightly increased signal intensity compared to the adjacent cortical bone on T1-weighted, T2-weighted, and STIR sequences. MRI also revealed enlargement of the TIOM at the exostosis site in one case. Histopathology performed either after surgical removal of the exostosis (one case) or post-mortem (one case) has demonstrated that the exostosis was composed of trabecular bone bordered by fibrous periosteum.

\section{Discussion/Conclusions:}

Exostoses of the palmar/plantar cortex of the Mc3/Mt3 bones should be considered as a potential cause of lameness in horses, with a possible TIOM associated injury. As some lesions may be asymptomatic, the significance of imaging findings should be confirmed by specific diagnostic analgesia. 
dGEMRIC IN NORMAL THOROUGHBRED HORSE CADAVER METACARPOPHALANGEAL AND TARSOPHALANGEAL JOINTS

A. Carstens $^{1}$, E. Lammentausta ${ }^{2,4}$, M. Velleman ${ }^{3}$, L. Dahlberg ${ }^{4}$, L. Prozesky ${ }^{5}$, J.C Jordaan ${ }^{6}$, R.M. Kirberger ${ }^{1} .{ }^{1}$ Section Diagnostic Imaging, ${ }^{2}$ Department of Diagnostic Radiology, Oulu University Hospital, Oulu, Finland, ${ }^{3}$ Little Company of Mary Hospital, Pretoria, South Africa, ${ }^{4}$ Joint and Soft Tissue Unit, Department of Clinical Sciences, Malmö, Lund University, Sweden, ${ }^{5}$ Section Pathology, Faculty of Veterinary Science, ${ }^{6}$ Departmen of Statistics, University of Pretoria, South Africa.

\section{Introduction/Purpose:}

Lameness is the primary cause of poor performance in horses, the metacarpo-/tarsophalangeal (MCP/MTP) joints being most often affected, ultimately resulting in osteoarthritis (OA). The effects of OA can be minimized if recognized early. Delayed gadolinium-enhanced MRI of cartilage (dGEMRIC) utilizes the negative charge of Gd-DTPA ${ }^{2-}$ which, when injected intra-articularly will diffuse more into cartilage which has a relatively high positive charge (i.e., that which has a decreased proteoglycan content), than that of a more normal cartilage. Cartilage damage can therefore be diagnosed earlier than when utilizing radiography. This study determines the $\mathrm{T} 1$ relaxation times (T1) of five different sites in the distal $\mathrm{Mc} 3 / \mathrm{Mt} 3$ condyle cartilage, evaluated after intra-articular injection of Gd-DTPA ${ }^{2-}$ into the MCP and MTP joints.

Methods:

The MCP and MTP joints of six Thoroughbred racehorses (3-6 years) with no evidence of lameness were harvested post euthanasia and scanned using a 1.5-T MRI machine (Siemens Avanto) and a head/neck coil. T1 was measured using an inversion recovery turbo spin-echo sequence with six inversion times (100-2800 ms) pre-Gd and at 30,60 120 , and 180 min post intra-articular injection of Gd-DTPA ${ }^{2-}$ (Magnevist $^{\text {T M }}$ ). A dedicated in-house MATLAB application (Mokkula) was used to calculate the T1 at five sites in the distal MCP/MTP condylar cartilages and Friedman's ANOVA and Wilcoxon signed-rank tests were performed to compare the differences between related groups.

Results:

The table shows the mean (SD) T1 of the limbs (in ms) for each of the five sites as well as the results of Friedman's ANOVA. Significant differences at the $5 \%$ level are marked by "*." All five sites showed significant differences between the pre-Gd and 30,60 , and 120 ms. Sites 1, 3, 4, and 5 showed significant differences between pre-Gd and $180 \mathrm{~ms}$ post intra-articular injection.

\begin{tabular}{|c|c|c|c|c|c|c|c|c|c|c|}
\hline & \multicolumn{2}{|l|}{ Site 1} & \multicolumn{2}{|l|}{ Site 2} & \multicolumn{2}{|l|}{ Site 3} & \multicolumn{2}{|l|}{ Site 4} & \multicolumn{2}{|l|}{ Site 5} \\
\hline & Mean & SD & Mean & SD & Mean & SD & Mean & SD & Mean & SD \\
\hline Pre & 985.0 & 143.8 & 837.7 & 140.3 & 1083.1 & 68.0 & 1135.1 & 45.8 & 892.9 & 99.4 \\
\hline 30 & 697.5 & 83.9 & 647.7 & 125.6 & 687.1 & 33.4 & 665.8 & 34.6 & 668.9 & 121.3 \\
\hline 60 & 660.7 & 88.3 & 646.5 & 123.0 & 676.6 & 58.3 & 670.4 & 70.0 & 622.6 & 132.6 \\
\hline 120 & 651.3 & 141.2 & 651.4 & 134.9 & 647.2 & 57.9 & 653.7 & 73.7 & 633.4 & 167.4 \\
\hline 180 & 676.1 & 155.7 & 695.6 & 161.5 & 660.1 & 147.0 & 657.2 & 148.5 & 605.7 & 145.7 \\
\hline $\begin{array}{l}\text { Friedman's } \\
\text { ANOVA }\end{array}$ & \multicolumn{2}{|c|}{$P<0.001^{\star}$} & \multicolumn{2}{|c|}{$P<0.003^{\star}$} & \multicolumn{2}{|c|}{$P<0.001^{*}$} & \multicolumn{2}{|c|}{$P<0.003^{\star}$} & \multicolumn{2}{|c|}{$P<0.001^{*}$} \\
\hline
\end{tabular}

\section{Discussion/Conclusions:}

dGEMRIC scans in the horse cadaver MCP/MTP joints should be conducted between 60 and 120 min post intra-articular Gd injection. A limitation of this study was the sample size. Further research is required on the effect of cooling, freezing, exercise, and intravenous administration of Gd on dGEMRIC in normal cadaver joints, as well as in joints with varying degrees of $\mathrm{OA}$.

\section{ULTRASONOGRAPHY OF THE LONG AND SHORT PARTS OF THE EQUINE TARSOCRURAL COLLATERAL LIGAMENT}

R. Hagen ${ }^{1}$, J.M. Kümmerle ${ }^{2}$, M. Kummer ${ }^{2} .{ }^{1}$ Department for Small Animals, Division of Diagnostic Imaging, ${ }^{2}$ Equine Department, Vetsuisse Faculty, University of Zürich, Zürich, Switzerland

Introduction:

Tarsocrural collateral ligament (functional) anatomy and the appearance of collateral ligament injury as detected by different imaging modalities have been described in the literature.
The aim of this study was to determine the sonographic appearance especially of the short parts of the tibiotarsal collateral ligament in both extended and flexed limbs. Attention was given as to whether examination in either extension or flexion would change the appearance of and/or aid identification and assessment of the individual ligaments.

\section{Methods:}

Seven pairs of tarsi of horses that had been euthanized for reasons other than hind limb lameness were isolated and prepared for sonography. Ultrasonography was performed using a linear high-frequency transducer. The long and short parts of the medial and lateral collateral ligaments of each specimen were examined in extension and flexion. Criteria for evaluation were visibility of origin, body and insertion of each individual ligament according to visibility of organized fibers and clear margins. Images were recorded of the origin, the body, and the insertion of each ligament and analyzed.

Results:

All the long collateral ligaments $(n=28)$ were imaged in all joints left and right, medially and laterally, and in extension and flexion. Overall, the three parts of the short collateral ligament were visualized more easily on the medial aspect of the limb, both in extension $76.6 \%$ and in flexion $87.8 \%$. On the lateral aspect, $59.6 \%$ of the short collateral ligament was visualized in flexion $87.8 \%$. On the lateral aspect, $59.6 \%$ of the short collateral ligament was visualized
in extension and only $54.4 \%$ in flexion. The middle part of the short collateral ligament was most consistently entirely imaged; in flexion $90 \%$ medially and $92 \%$ laterally and in extension $94.6 \%$ medially and $83.6 \%$ laterally. The profound part of the short collateral ligament was most difficult to visualize, its mid-part was imaged in $83.5 \%$ on the medial aspect in flexion. The mid-part of the short collateral ligament showed mild torsion of its fibers in extension (when it is relaxed) and parallel fiber arrangement in flexion. Imaging was hampered in some cases by subcutaneous and intrafascial gas accumulation due to the preparation of the specimens.

Conclusions:

Ultrasonographic examination allows identification of the individual subsections of the shor tarsocrural collateral ligament. The long collateral ligament is comfortably identified and completely imaged. Flexion of the tarsus helps to identify the individual parts of the short collateral ligaments, especially on the medial aspect of the tarsus. It may be beneficial to scan the tarsocrural collateral ligaments in flexion especially if injury to the short part is scan the tarsocrural collateral ligaments in flexion especially if injury to the short part is
suspected. Whether this applies to the live equine patient and to injured collateral ligaments too is currently under investigation.

\section{ULTRASONOGRAPHY IS SUPERIOR TO RADIOGRAPHY FOR THE DETECTION OF SUBCHONDRAL BONE CHANGES IN THE MEDIAL FEMORAL CONDYLE OF HORSES}

C. Gerdes, M.J. Head. Rossdales Diagnostic Centre, Newmarket, UK

Introduction:

Radiography is widely considered to be the optimal imaging method for detection of subchondral bone changes affecting the medial femorotibial joint of horses. Recent experience has suggested that ultrasonographic evaluation provides more information than radiography and is more sensitive in detecting lesions that may be overlooked if radiography alone is employed.

Methods:

A retrospective evaluation of 28 horses diagnosed with subchondral bone changes affecting the medial femoral condyles (MFCs) was performed. Inclusion in the study necessitated that the horse had been assessed radiographically with diagnostic quality images and unthat the horse had been assessed radiographically with diagnostic quality images and un-
dergone ultrasonographic assessment of the MFCs, performed with the hindlimb flexed dergone ultrasonographic assessment of the MFCs, performed with the hindlimb flexed
using a high-frequency transducer, by an experienced ultrasonographer (MJH in all cases). Anonymized images were reviewed by $\mathrm{MJH}$ : caudocranial radiographic projections were graded according to a modified version of a scale published previously ${ }^{1}$; longitudinal and transverse ultrasonographs were reviewed and graded according to a scale developed by the authors. Each technique for each case was therefore assigned a grade from 0 (normal) the authors. Each technique for each case was therefore assigned a grade from 0 (normal)
to 4 (most severe abnormality). Method agreement was assessed using Cohen's kappa statistic with $95 \%$ confidence intervals. Comparison between methods was performed using Fisher's exact test.

Results:

In relation to the results obtained by the two different methods, agreement was observed in 11 of 28 cases $(39.3 \%)$. Cohen kappa was 0.21 , showing only fair agreement. There was a statistically significant $(P=0.01)$ difference in the detection of lesions between methods: subchondral bone changes were detected in $28 / 28$ of horses (100\%) when using ultrasound compared to $21 / 28(75 \%)$ with radiography.

Conclusions:

Ultrasonographic assessment of the MFC is a simple, easily performed technique that provides a more sensitive method for detection of subchondral bone damage affecting the equine stifle than radiography. It should be included as part of the standard diagnostic evaluation of horses with lameness associated with the stifle.

Reference:

1. Steinheimer, DN, Mcllwraith, CW, Park, RD, Steyn, PF. Vet Radiol Ultrasound 1995;36:478-484 


\section{OBLIQUE VIEW EQUINE STIFLE SCINTIGRAMS: STANDARDIZATION AND EVALUATION OF A METHOD}

M. Grapperon-Mathis, C. Ley, M. Berger, K. Hansson. Section of Diagnostic Imaging, University Animal Hospital and University of Agricultural Sciences, Uppsala, Sweden

\section{Introduction:}

Equine stifle lesions are frequent causes of lameness and often occur in the medial regions of the joint. Bone phase scintigraphy is a well-established imaging modality used to investigate equine musculoskeletal problems and lateral and caudal views of the stifle are well described. Considerable attenuation of medial stifle radiopharmaceutical uptakes occurs in these views due to the thick tissues of the stifle region, and this limits the accuracy and th quality of the diagnosis. No standardized method for the reliable and repeatable positioning of the lateral view of the stifle has been described. The aims of this project were (1) to establish a repeatable and reliable method to obtain "true" lateral view of the stifle joint and (2) to evaluate qualitatively and quantitatively images of femorotibial medial phantoms using standardized lateral and oblique views. standardized

Twenty horses referred for scintigraphic investigation of lameness were used. True latera images of the stifle were repeatedly acquired when medial and lateral radioactive poin sources, located at the origin of the femorotibial collateral ligaments, were aligned in a vertical line profile. The angle between the axis of the foot and the axis of the gamma camera was measured for each acquisition and compared with the angle obtained when an experienced operator subjectively positioned the gamma camera. Following this, two additional point sources were put at the level of the medial and caudal joint margins and three views were acquired: true lateral, $30^{\circ}$ caudolateral and $30^{\circ}$ craniolateral oblique. The images were assessed quantitatively for intensity and qualitatively for detectability.

Results:

For each horse, the measured angles of the lateral view did not significantly differ from the angle obtained by the experienced operator positioning. The conspicuity of the medial point sources was improved on the $30^{\circ}$ caudolateral position. The caudal joint point source had the highest relative intensity in the $30^{\circ}$ caudolateral oblique views whereas it had the lowest in the lateral views. The $30^{\circ}$ craniolateral view was not advantageous when compared with a straight lateral projection.

Discussion/Conclusions:

Objective positioning of the gamma camera relatively to the limb to obtain a true lateral view of the stifle did not show any significant difference when compared with experienced operator positioning. However, the establishment of this lateral position was necessary to define the usefulness of oblique images. Further investigations on the reliability of the objective positioning method are warranted. The $30^{\circ}$ caudolateral oblique view may be very useful in identifying increases in radiopharmaceutical uptake in the medial regions of the equine stifle.

\section{EFFECT OF RESPIRATORY PHASE ON RADIOLOGICAL THORACIC DIMENSIONS IN NORMAL THOROUGHBRED HORSES}

A. Carstens ${ }^{1}$, R.M. Kirberger ${ }^{1}$, J.C. Jordaan ${ }^{2} .{ }^{1}$ Section Diagnostic Imaging, Faculty of Veterinary Science, ${ }^{2}$ Department of Statistics, University of Pretoria, Onderstepoort 0110 , South Africa

\section{Introduction/Purpose:}

Respiratory conditions causing poor performance in horses are usually as a result of uppe respiratory diseases or of pulmonary origin. Certain thoracic dimensions, such as the spinotracheal angle (STA), the angles of the diaphragmatic crura and cupula, the dimensions of the caudal vena cava (CVC), and the distance between the heart and diaphragm may change in horses with dyspneoa and respiratory disease.

Methods:

Standing lateral thoracic radiographs of the thorax of 15 Thoroughbred horses, 3-6 years old, with no evidence of respiratory disease, were made at peak inspiration and end expiration. The spinotracheal angle (STA) was measured as well as the angle of the most crania diaphragmatic crus to the thoracic vertebrae (Vert-CrusA). The angle between the crus and cupula was calculated (Crus-CupA), by measuring the angles of the most cranial diaphragmatic crus and the ventral aspect of the diaphragmatic cupula to the horizontal plane. The CVC height at the caudal cardiac silhouette (CVC-ht-Cardiac) and at the diaphragm (CVC-ht-Diaphr) was measured as well as the dorsal and ventral CVC lengths (CVC-lengthDors/CVC-length-Ventr). The dorsal CVC lengths could not be consistently measured and were therefore discarded. The distance between the cardiac silhouette and the diaphragm (CardiacS-Diaphr) was measured at the level of the ventral CVC. Paired $t$-tests were used to determine significance of difference between the means of expiratory and inspiratory measurements with significance set at $P<0.05$.

Results:

The table shows the mean $( \pm S D)$ values of the parameters measured. Significant differences between expiratory and inspiratory values are marked by "*."

\begin{tabular}{|c|c|c|c|c|c|c|c|c|c|c|c|c|c|}
\hline \multicolumn{2}{|l|}{$\begin{array}{l}\text { STA } \\
\left({ }^{\circ}\right) \\
\end{array}$} & \multicolumn{2}{|c|}{$\begin{array}{l}\text { Vert-CrusA } \\
\left({ }^{\circ}\right) \\
\end{array}$} & \multicolumn{2}{|c|}{$\begin{array}{l}\text { Crus-CupA } \\
\left({ }^{\circ}\right)\end{array}$} & \multicolumn{2}{|c|}{$\begin{array}{l}\text { CVC-ht- } \\
\text { Cardiac } \\
(\mathrm{mm})\end{array}$} & \multicolumn{2}{|c|}{$\begin{array}{l}\text { CVC-ht- } \\
\text { Diaphr } \\
(\mathrm{mm}) \\
\end{array}$} & \multicolumn{2}{|c|}{$\begin{array}{l}\text { CVC- } \\
\text { length } \\
\text { Ventr } \\
(\mathrm{mm}) \\
\end{array}$} & \multicolumn{2}{|c|}{$\begin{array}{l}\text { CardiacS- } \\
\text { Diaphr } \\
(\mathrm{mm}) \\
\end{array}$} \\
\hline Exp & Insp & Exp & Insp & Exp & Insp & $\overline{\operatorname{Exp}}$ & $\operatorname{lnsp}$ & $\overline{E x p}$ & $\operatorname{Insp}$ & Exp & Insp & Exp & Insp \\
\hline $\begin{array}{l}7.7 \\
\pm \\
2.8\end{array}$ & $\begin{array}{l}9.5 \\
\pm \\
2.9\end{array}$ & $\begin{array}{l}34.2 \\
\pm \\
3\end{array}$ & $\begin{array}{l}36.2 \\
\pm \\
3.4\end{array}$ & $\begin{array}{l}112.7 \\
\pm \\
6\end{array}$ & $\begin{array}{l}116.2 \\
\pm \\
7.5\end{array}$ & $\begin{array}{l}4.8 \\
\pm \\
0.6\end{array}$ & $\begin{array}{l}4.7 \\
\pm \\
1.5\end{array}$ & $\begin{array}{l}4.3 \\
\pm \\
0.7\end{array}$ & $\begin{array}{l}4.3 \\
\pm \\
0.4\end{array}$ & $\begin{array}{l}4.9 \\
\pm \\
1.3\end{array}$ & $\begin{array}{l}5.6 \\
\pm \\
1.2\end{array}$ & $\begin{array}{l}3.2 \\
\pm \\
0.8\end{array}$ & $\begin{array}{l}3.9 \\
\pm \\
1\end{array}$ \\
\hline \multicolumn{2}{|c|}{$\begin{array}{l}P< \\
0.012^{*}\end{array}$} & \multicolumn{2}{|c|}{$\begin{array}{l}P<0.01^{*} \\
\text { (one- } \\
\text { tailed) }\end{array}$} & \multicolumn{2}{|c|}{$P<0.125$} & \multicolumn{2}{|c|}{$P<0.728$} & \multicolumn{2}{|c|}{$P<0.766$} & \multicolumn{2}{|c|}{$P<0.111$} & \multicolumn{2}{|c|}{$\begin{array}{l}P< \\
0.014^{*}\end{array}$} \\
\hline
\end{tabular}

\section{Discussion/Conclusions:}

Radiologically, there is a significant difference in the STA, the Vert-CrusA and the distance between the cardiac silhouette and the diaphragm of the normal Thoroughbred horses at inspiration and expiration. This implies that the cranial thoracic trachea moves ventrally during inspiration, the cranial crus of the diaphragm (likely the left crus) moves into a more vertical caudal position during inspiration and the diaphragm moves caudally relative to the heart during inspiration. This study in normal horses may serve as a reference when radiologically evaluating cases such as recurrent airway obstruction or URT, where these dimensions may differ significantly due to differences in airway resistance, biomechanics, and dyspnoea.

\section{MAGIC ANGLE MAGNETIC RESONANCE IMAGING OF NATURALLY OCCURRING LESIONS IN EQUINE TENDONS} $\frac{\text { M. Spriet, B. Murphy, E.R. Wisner. School of Veterinary Medicine, University of California, }}{\text { Davis, CA }}$

Introduction/Purpose:

Magic angle magnetic resonance (MR) imaging consists of taking advantage of the in creased signal intensity present in tendons imaged at $55^{\circ}$ relative to the magnetic field. Studies in human patients have shown that magic angle MR imaging is valuable for detection of chronic tendon lesions. Previous work on laser-induced lesions in equine tendons has confirmed the potential of this MR technique for lesion detection. Magic angle MR imaging confirmed the potential of this MR technique for lesion detection. Magic angle MR imaging
also allows calculation of the T1 value of tendons. Increased T1 values have been reported in people with chronic tendinopathy. The T1 values of normal equine tendons have been reported but there are currently no available data for abnormal equine tendons. The firs objective of our study was to compare the appearance of naturally occurring equine tendon lesions on magic angle MR imaging and conventional MR imaging. The second objective of our study was to determine $\mathrm{T} 1$ values of abnormal equine tendons.

Methods:

Six limbs with naturally occurring tendon lesions were collected from horses euthanized for reasons unrelated to the study. Four limbs had lesions of the superficial digital flexor tendon (SDFT) and/or deep digital flexor tendon (DDFT) in the metacarpal area and two limbs had lesions of the DDFT in the pastern/foot area. The limbs were imaged with a 1.5-T MR scanner using a surface general-purpose flexible coil. The limbs were imaged first with the tendons parallel to the magnetic field $\left(0^{\circ}\right)$ using sagittal T1-weighted and transverse T1-weighted, T2-weighted, and STIR sequences. Imaging with T1-w sagittal and transverse sequences was then repeated with the tendons positioned at $55^{\circ}$ relative to the magnetic field. Transverse proton density and inversion recovery sequences were also obtained in the metacarpal area at $55^{\circ}$ for T1 measurement. The conventional $\left(0^{\circ}\right)$ and magic angle images were reviewed independently for the presence and imaging features of the lesions. The $\mathrm{T} 1$ values were calculated according to a previously described method. All lesions were examined histologically after formalin fixation and paraffin embedding.

Results:

All abnormal areas were correctly identified with both techniques, however the appearance of lesions differed with acute tendon lesions better visualized with conventional imaging and mineralization and neovascularization best seen with magic angle imaging. Increased T1 values were observed in all abnormal tendons, even in areas where no focal lesions were identified.

\section{Discussion/Conclusions:}

Although magic angle imaging did not improve lesion detection compared to conventional imaging, it contributed to better characterize the lesions. Similar to what has been reported in people, an increased T1 value is observed in horses with chronic tendinopathy. The increased T1 value might reflect diffuse changes in the biochemical composition of tendons, even with focal lesions. Further work is needed to correlate the changes in T1 value and the composition of the tendon. 


\section{ELASTOGRAPHIC EVALUATION OF NORMAL TENDONS AND LIGAMENTS OF THE EQUINE DISTAL FORELIMB}

G.S. Seiler, M. Lustgarten, W.R. Redding. North Carolina State University, Raleigh, NC

\section{Introduction/Purpose:}

Elastography is an ultrasound technique that evaluates the stiffness of tissues by measuring the displacement of ultrasound echoes before and after compression. Elastography thereby provides information about the mechanical properties of tissues and is currently used in people to differentiate malignant from benign lesions in breast, prostate, and thyroid tissue. Elastographic evaluation of musculoskeletal structures, particularly the human Achilles tendon, provides information enabling more accurate diagnosis of minor injuries and early tendinitis. The purpose of this study was to evaluate feasibility and repeatability of this method for imaging of the equine distal forelimb, as well as to establish the norma elastographic appearance of the equine digital flexor tendons and suspensory ligament branches.

Methods:

Horses with no evidence of forelimb lameness at the trot, and with sonographically norma tendons and ligaments were included. Elastographic images of the superficial and deep digital flexor tendons, and the branches of the suspensory ligament were obtained in longitudinal and transverse planes. These measurements were also performed with the leg in a nonweight bearing (flexed) position. A second ultrasonographer then repeated the examination. Images were evaluated using qualitative and quantitative methods. A qualitative assessment of softness was scored as $1-4(1=$ hard; $4=$ soft $)$ on an image where tissue

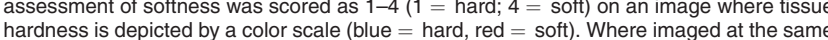
depth, a ratio of stiffness between the superficial and deep digital flexor tendons was calculated (MyLab 70, Biosound Esaote Inc. Indianapolis, IN). Weight bearing and nonweigh bearing scores were compared using a paired Student's $t$-test.

Results:

Eleven forelimbs of nine horses were included in the study. Normal weight-bearing tendons and ligaments were hard to intermediate in stiffness (1.26, SD 0.33), whereas nonweight bearing tendons and ligaments were significantly softer $(1.72$, SD 0.59$)(P<0.001)$. No significant differences were found between measurements obtained by the two observer $(P=0.14)$. The digital flexor tendons became progressively harder from proximal $(1.80$ SD 0.29 ) to distal $(1.30, S D 0.41)$, the superficial aspects of the suspensory branches were softer (1.97, SD 0.81) than the deep aspects (1.58, SD 0.77). The peritendinous tissues were consistently soft. The average ratio of stiffness of the deep digital flexor tendon to the superficial digital flexor tendon was 0.978 (SD 0.166).

superficial digital flexor tendon

We have found that elastography is a feasible, noninvasive and repeatable method fo evaluation of the equine superficial and deep digital flexor tendons, and the branches of the suspensory ligaments. The normal elastographic appearance of tendons and ligaments of the equine distal forelimb can be used as a baseline to investigate equine tendon and ligament injuries. Elastography complements conventional grayscale ultrasound by providing information about stiffness and therefore strength and integrity of the fiber structure.

\section{INTRA- AND INTEROBSERVER AGREEMENT OF RADIOGRAPHIC STUDIES FOR CERVICAL FACET JOINT OSTEOARTHRITIS IN HORSES}

S.E. Allen, C.S. Avella, R. Weller. Department of Veterinary Clinical Sciences, The Royal Veterinary College, University of London, UK

\section{Introduction/Purpose:}

The aim of this study was to determine the repeatability of radiography and computed tomography (CT) in the diagnosis of cervical facet osteoarthritis in the horse. It was hypothesized that intraobserver agreement would be better than interobserver agreement and that agreement would be better for $\mathrm{CT}$ than for radiographs.

\section{Methods:}

Radiographs and CT scans of 125 pairs of facet joints (from 28 equine cadaver necks) were evaluated by two observers on two occasions using a newly developed grading system. Intra and interobserver agreement was quantified using the kappa statistic.

Results:

Moderate to substantial $(k=0.42-0.78)$ intra and interobserver agreement was found on radiographs and $\mathrm{CT}$ for deciding on the presence or absence of osteoarthritis of cervical face joints. Agreement for deciding on the radiographic signs of osteoarthritic lesions present was similar on radiographs and CT (moderate to substantial; $k=0.42-0.75$ ). However interobserver agreement was slight on radiographs $(k=0.11)$ and fair on CT $(k=0.40)$ Discussion/Conclusion:

It has been demonstrated that laterolateral radiographs and CT have an acceptable repeatability for diagnosing osteoarthritis of the cervical facet joints, even if the agreement on specific radiographic signs is poor to moderate. Laterolateral radiographs and CT have similar precision for determining if osteoarthritis is present on cervical facet joints, suggesting that superimposition does not play major role in determining agreement. Further studies will evaluate the accuracy of radiography and CT by comparing it to postmortem findings as gold standard.

\section{QUANTITATIVE ASSESSMENT OF NUCLEAR BONE SCINTIGRAPHY USING THE} REGION OF INTEREST TECHNIQUE AS APPLIED TO THE CAUDAL CERVICAL SPINE

K. Gerlach, M. Keyl, W. Brehm. Large Animal Clinic for Surgery, Faculty of Veterinary Medicine, University of Leipzig, Germany

\section{Introduction/Purpose:}

The interpretation of scintigraphic examination of the equine cervical spine is discussed differently due to the physiological pattern of radiopharmaceutical uptake, particularly with regards to the caudal articular facet joints. Some authors assumed that measurable activity of the vertebrae decreases caudally, based on increased but individually diverse neck muscle thickness. Others reported that the increased pharmaceutical uptake of the caudal facet joint has to be considered as physiological, occurring more frequently in some breeds. The aim of this study was to quantify bone scans and to determine standard ranges of radiopharmaceutical uptake. The second intention was to carry out the test of correction of muscle thickness at the level of the caudal facet joints.

Methods:

Thirty-one horses, all of them showing clinically inconspicuous cervical spines, were examined The articular facet joints $\mathrm{C} 3 / \mathrm{C} 4$ to $\mathrm{C} 7 / \mathrm{Th} 1$ and the vertebral body of $\mathrm{C} 6$, which served as 'regions of interest, were put into relation to different regions of reference in order to create ratios of uptake. The vertebral bodies of $\mathrm{C} 3$ and $\mathrm{C} 4$, as well as the facet joint $\mathrm{C} 3 / \mathrm{C} 4$, which already served as a region of interest, were tested as regions of reference. Furthermore, standard ranges for the ratios of uptake were determined. After sonographical measurement of the muscle thickness in a horizontal direction to the articular facet joints, the uptake ratios of these joints were corrected toward a zero value using a mathematical formula. Likewise, standard ranges were assigned for the corrected values.

Results:

The results showed that the uptake ratios scattered over a wider range after correction for muscle thickness compared to the native values. This results in larger and therefore more imprecise standard ranges. Accordingly, the native values are preferred. Due to the fact that most radiological changes are found in these facet joints, the standard ranges of the native values of $\mathrm{C} 5 / \mathrm{C} 6$ and $\mathrm{C} 6 / \mathrm{C} 7$ should be pointed out. They constitute 0.82 1.10 for the facet joint $\mathrm{C} 5 / \mathrm{C} 6$ and $0.75-1.23$ for the facet joint $\mathrm{C} 6 / \mathrm{C} 7$ with reference area $\mathrm{C} 3 / \mathrm{C} 4$

\section{Discussion/Conclusions:}

In summary, it proves feasible to quantitatively assess the equine cervical spine by using the regions of interest technique, but correction of muscle thickness is not necessary. As this study shows, acceptable standard ratios can be determined for the articular facet joints $\mathrm{C} 3 / \mathrm{C} 4$ to $\mathrm{C} 7 / \mathrm{Th} 1$ and the vertebral body of $\mathrm{C} 6$.

\section{MAGNETIC RESONANCE ANGIOGRAPHY OF THE NORMAL EQUINE HEAD}

G. Manso-Díaz ${ }^{1}$, I. García-Real ${ }^{1}$, C. Casteleyn ${ }^{2}$, O. Taeymans ${ }^{3} .{ }^{1}$ Department of Anima Medicine and Surgery, School of Veterinary Medicine, Complutense University, Madrid, Spain, ${ }^{2}$ Department of Morphology, Faculty of Veterinary Medicine, Ghent University, Belgium, ${ }^{3}$ Department of Clinical Sciences, Cummings School of Veterinary Medicine, Tufts University, North Grafton, MA

Introduction:

Digital subtraction angiography (DSA) is a common imaging technique for the evaluation of blood vessels. However, magnetic resonance angiography (MRA) and computed tomography angiography (CTA) are gradually replacing this technique. The use of contrast agents is essential for performing DSA and CTA, making these techniques very expensive in equine patients, in whom it would require large volumes of contrast. The aim of this study was to provide a comprehensive anatomical reference of the normal equine head vasculature using a noncontrast MRA technique, as well as to describe the potential clinical applications of this technique.

\section{Materials and Methods:}

Four healthy adult horses (two Thoroughbreds, one Andalusian, and 1 mixed breed) were used for the study. MR studies were performed with a 0.23-T magnet (Philips Medical System) with the horses lying in right recumbency under general anesthesia. A body receiver coil was covering the area of interest. Imaging protocol included a sagittal turbo spin-echo T1-weighted sequence, used to depict landmarks, followed by a transverse time-of-flight (TOF Inflow MRA2D) sequence. This protocol was repeated five times, in order to obtain images of the entire head. Landmarks demarcating the caudal limit of each field of view wer the foramen magnum, the cranial border of the hypophysis, the cranial part of the middle nasal concha, the third premolar, and the mandibular symphysis. Data were processed using maximum-intensity projection. The vessels were identified in the postprocessed images using slice-by-slice technique, compared with images illustrated in anatomical textbooks, and using vascular corrosion casts.

and using

The imaging protocol provided excellent image contrast and resolution, allowing visualization of all major intracranial and extracranial vessels down to a size of approximately $2 \mathrm{~mm}$ in diameter. This resulted into identification of vessels to the order of third to fourth branches. The visibility of the arteries was higher than the veins, which showed lower signal intensity. The average total image acquisition time per horse was $60 \mathrm{~min}$.

Conclusions:

The use of a specific vascular sequence such as TOF Inflow MRA2D allows good visualization of the equine head vasculature, and eliminates the need of contrast media. The bette visibility of the arterial system was resulting from its high flow velocity resulting in increased signal intensity. Moreover, the lower flow velocity of the venous system implies a greate percentage of stationary protons exposed to repeated excitation pulses, causing a loss of signal as a result of saturation. This imaging technique was deemed suitable for clinical use, and may be helpful in conditions such as guttural pouch mycosis or surgical planning of facial masses. 


\section{USE OF SCINTIGRAPHY FOR IN VIVO TRACKING OF EQUINE MESENCHYMAL STEM CELLS}

M. Spriet, A. Sole, L.D. Galuppo, K.A. Padgett, D.L. Borjesson, E.R. Wisner, R.J. Brosnan M.A. Vidal. School of Veterinary Medicine, University of California, Davis, CA

\section{Introduction/Purpose:}

Mesenchymal stem cells (MSCs) have been used recently for treatment of musculoskeletal lesions in the horse Clinical trials have reported successful outcomes but little is known about the fate of the cells after injection. Determining the localization and persistence of the MSCs after injection is a key element for optimization of treatment. Labeling of stem cells with ${ }^{99 m}$ Technetium-Hexamethyl-Propylene-Amine-Oxime (Tc-HMPAO) for scintigraphic cell tracking has been proven useful for evaluation of the distribution and quantification of stem cells in rodent models and human clinical trials but tracking of labeled equine MSCs has not been reported. The purpose of our study was to label equine MSCs with TC-HMPAO and use planar scintigraphy to compare different routes of MSC administration into the equine distal limb.

\section{Methods:}

MSCs were isolated from bone marrow collected from a donor horse. Approximately, $8 \times$ $10^{7} \mathrm{MSC}$ were added to $2.5 \mathrm{ml}$ of a solution containing $250 \mu \mathrm{g}$ of HMPAO, $7.6 \mu \mathrm{g}$ of tin (II) chloride and $370 \mathrm{MBq}(10 \mathrm{mCi})$ of technetium pertechnetate. After $23 \mathrm{~min}$ of incubation at room temperature, the MSCs were isolated by centrifugation, washed and resuspended in saline solution. Cell viability was assessed by trypan blue exclusion test immediately after labeling. Labeling efficiency and labeling persistence at $6 \mathrm{~h}$ were calculated. Labeled cells were injected into six different horses using both intra-arterial and intravenous regional limb perfusion. Static and dynamic planar scintigraphic images were acquired at the time of injection and up to $24 \mathrm{~h}$ postinjection.

Results:

The viability of the cells after labeling (88\%) was similar to the viability before labeling $(90 \%)$. The labeling efficiency ranged from 29.8 to $42.4 \%$ with a mean of $35.7 \%$. A labeling persistence of $83 \%$ was measured $6 \mathrm{~h}$ after labeling. Good quality images were obtained on all horses up to $6 \mathrm{~h}$ after injection. In three of the horses, images of good quality wer also obtained $24 \mathrm{~h}$ after injection.

Discussion/Conclusions:

Labeling of equine MSCs with Tc-HMPAO is well tolerated by the cells. The labeling efficiency and labeling persistence are similar to what has been reported with rodent MSCs. Using this protocol, tracking of cell distribution and quantification can easily be performed up to $6 \mathrm{~h}$ after injection. In order to improve the quality of the tracking of the cells up to $24 \mathrm{~h}$, increasing the dose of technetium might be helpful.

\section{VENOUS ANEURYSM OF THE RIGHT EXTERNAL JUGULAR VEIN IN A DOG}

D. Della Santa ${ }^{1}$, F. Rossi ${ }^{2}$, P. Gagliardi ${ }^{1}$, M. Guardabassi ${ }^{1}$, R. Verin ${ }^{3}$, A. Poli ${ }^{3} .{ }^{1}$ Clinica Veterinaria Mugello, San Piero a Sieve (FI), ${ }^{2}$ Clinica Veterinaria dell'Orologio, Sasso Marcon (BO), Italy, ${ }^{3}$ Dipartimento di Patologia Animale, Facoltà di Medicina Veterinaria, Università di Pisa, Pisa, Italy

\section{Case History:}

An 8-month-old Rottweiler had a soft, nonpainful swelling over the right ventrolateral cervica region recently noted by the owner who did not report any previous trauma. Fine needle as piration biopsies of the lesion were not diagnostic because of hemodilution. Ultrasonography of the lesion showed a thin-walled ovoid-shaped collapsible lesion with cavitary appearance containing mildly echogenic fluid swirling inside. This structure was connected caudally with a large venous vessel (most likely the right external jugular vein); a valve-like structure was interposed between them. A vascular connection could not be clearly demonstrated cranially. Doppler study showed low-velocity pulsatile flow with cranial-to-caudal direction originating from the cranial aspect of the lesion. Dual-phase CT angiography was performed in order to confirm the vascular origin of the lesion and plan surgical intervention. Arterial in order to confirm the vascular origin of the lesion and plan surgical intervention. Arterial
phase was normal: no arteriovenous communication was found. The soft-tissue swelling phase was normal: no arteriovenous communication was found. The soft-tissue swelling extending from the midcervical region to the thoracic inlet. The right omobrachial vein was joining the right jugular vein adjacent to the caudal border of the lesion. Remaining cervical vessels were within normal limits. Small multiple mineralizations were visible cranial to the right supraglenoid tubercle of the scapula, along the brachial biceps tendon. At surgery the lesion was exposed, the right jugular vein ligated just cranial to the dilated segment and the wall of the aneurysm excised. Histopathology and immunohistochemistry of the excised tissue confirmed the vascular origin of the lesion. Twelve months after surgery the patien was not showing any clinical signs.

Discussion:

Although a definitive classification does not exist, venous dilations in human medicine are classified based on macroscopic clinicopathologic findings as either "saccular venous aneurysms" if saccular in shape or "phlebectasias" if fusiform. Both jugular vein aneurysms and phlebectasias, although rare, do affect the jugular veins in people: there is a tendency for phlebectasia to occur in children (suspected to be congenital or to arise from a primary weakening of the muscular layer of the venous wall) and aneurysms in adults (often traumatic). Contrast-enhanced CT and color Doppler ultrasonography are the preferred methods of diagnosis by most investigators. In this case, dual-phase CT proved to be superior to ultrasound to demonstrate the exact size and topography of the lesion and to show the precise vascular connections. For phlebectasias, conservative treatment is usually recommended, whereas for saccular aneurysms, surgical resection is the treatment of choice because of the higher risk of thrombus formation. The presence of mineralizations in the region of the the higher risk of thrombus formation. The presence of mineralizations in the region of the
biceps tendon might suggest a trauma as cause of the aneurysm, but definitive etiology of the lesion could not be determined. To the best of our knowledge, this is the first report of a jugular vein aneurysm in the dog.

\section{COMPUTED TOMOGRAPHY (CT) FINDINGS IN A DOG WITH INTRACRANIAL HEMORRHAGE SECONDARY TO ANGIOSTRONGYLOSIS}

M. Zarelli, R. Shiel, B. Gallagher, C. Skelly, S. Cahalan, H. McAllister. School of Veterinary Medicine, University Veterinary Hospital, University College Dublin, Belfield, Dublin, Ireland

\section{Introduction/Purpose:}

Magnetic resonance imaging finding of dogs with intracranial hemorrhage secondary to coagulation defects associated with Angiostrongylus vasorum infection has been described. However, to the authors' knowledge, this is the first report of CT findings in a dog with intracranial hemorrhage secondary to angiostrongylosis.

\section{Materials and Methods:}

A 7-month-old female cocker spaniel dog was presented with a history of cough of 2 week's duration, acute onset lethargy, decreased responsiveness, and episodes of hyperexcitability. On physical examination, bilateral generalized increased lung sounds, bilateral episcleral hemorrhage, and systemic hypertension were detected. Hematology, thoracic radiographs, hemorrhage, and systemic hypertension were detected. Hematology, thoracic radiographs, thoracic, and precontrast and postcontrast brain comp test and post

Prolonged buccal mucosal bleeding time and elevated D-dimer concentrations were detected. Thoracic radiographs showed a generalized moderate interstitial lung pattern. Thoracic $C T$ revealed diffuse moderate hyperdense appearance to the walls of the bronchial tree and interstitium and diffuse areas of moderate bronchiectasis scattered throughout all lung lobes. The brain CT showed marked hyperdense areas that created mass effect shifting lung lobes. The brain CT showed marked hyperdense areas that created mass effect shifting
the midline. In addition, there were hyperdense and hypodense lesions of smaller dimenthe midline. In addition, there were hyperdense and hypodense lesions of smaller dimen-
sions scattered throughout the cerebral and cerebellar parenchyma. A zinc sulfate flotation test confirmed large numbers of $A$. vasorum L1 larvae. Despite therapy, the dog continued to deteriorate and was euthanatized. Postmortem examination confirmed the presence of multiple intracranial and extracranial hemorrhages.

Discussion:

A wide variety of clinical signs have been associated with $A$. vasorum infection, including neurological signs. Various diagnostic imaging modalities have been used to investigate and monitor Angiostrongylus infection. However, this is the first report of CT findings in a dog with neurological signs and intracranial hemorrhage secondary to angiostrongylosis. Conclusion:

Angiostrongylosis should be considered as a differential diagnosis in dogs with neurological signs and CT signs of intracranial hemorrhage.

References:

1. Chapman, P., Boag A., Guitian, J. \& Boswood, A. Angiostrongylus vasorum infection in 23 dogs (1999-2002). J Small Anim Pract 2004;45:435-440.

2. Enzmann, D., Britt, R., Lyons, B., Buxton, J. \& Wilson, D. Natural history of experimental intracerebral hemorrhage: sonography, computed tomography and neuropathology. Am $J$ Neuroradiol 1981:2:517-526.

3. Garosi, L., Platt, S., McConnell, J., Wray, J. \& Smith, K. Intracranial haemorrhage associated with Angiostrongylus vasorum infection in three dogs. J Small Anim Pract 2005;46:93- 


\section{PYOGENIC VENTRICULITIS IN A DOG}

$\underline{\text { H.J. Choi }}{ }^{1}$, J.H. Choi ${ }^{2}$, Y.W. Lee ${ }^{1}$, D.I. Jung ${ }^{3}$, H.C. Lee ${ }^{3} .{ }^{1}$ College of Veterinary Medicine Chungnam National University, Daejeon, ${ }^{2}$ College of Veterinary Medicine, Chonnam $\mathrm{Na}$ tional University, Gwangju, ${ }^{3}$ College of Veterinary Medicine, Gyeongsang National University, Jinju, South Korea.

\section{Case History:}

A 2-month-old intact female Jindo dog was referred with a 3-day history of seizure, tetraparesis, and hypersalivation 10 days after bite injury on the head region. The $\mathrm{CBC}$ revealed moderate neutrophilic leukocytosis, and skull radiography showed calvarial bony defect with an overlapped small bone fragment. The CT examination revealed a left parietal bone fracture with a laterally deviated bone fragment and soft-tissue swelling. Contrast-enhanced CT scans showed asymmetric hydrocephalus and remarkable ependymal enhancement in lateral and third ventricles, and soft-tissue densities, most likely representing inflammatory debris, were occupying much of the dependent portions of the lateral ventricles.
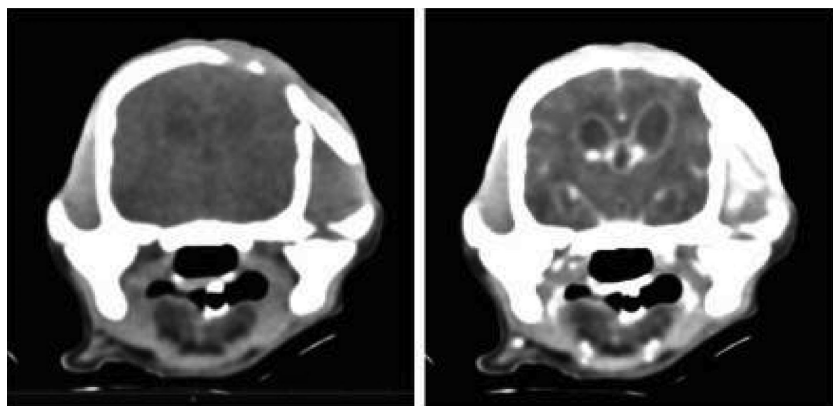

Neutrophilic pleocytosis and intracellular bacteria were detected on CSF analysis. Antimicrobial susceptibility test showed a growth of Pateurella multocida. Based on the results of these examinations, pyogenic ventriculitis induced by bite injury was suspected. The dog died after CT scan, and the greenish fluid was in lateral ventricles at necropsy. Histopathologic examinations confirmed the severe pyogenic ventriculitis with meningoencephalitis. Discussion:

Pyogenic ventriculits is relatively rare and a known complication of pyogenic meningitis, which is most often characterized by ependymal inflammation with the accumulation of intraventricular pus. Detection of ventricular debris and ependymal enhancement were considered as important signs of ventriculitis on CT images. Bite wound of the head might have been the cause of ventriculitis in this case.

\section{PORENCEPHALY IN DOGS AND CATS: MAGNETIC RESONANCE IMAGING} FINDINGS AND CLINICAL SYMPTOMS

M.J. Schmidt, S. Klumpp, K. Amort, M. Kramer. Klinik für Kleintiere-Chirurgie, Justus-Liebig Universität, Gießen, Germany

\section{Background:}

Young and adult dogs and cats can develop a variety of clinical signs due to porencephalic forebrain defects. These symptoms and the underlying magnetic resonance imaging (MRI) defects have not been described yet.

Objective:

To present clinical symptoms and MRI features of brain malformations in five dogs and two cats characterized by extensive cystic lesions of the cerebral hemispheres presented as porencephaly and to describe the variable clinical expression of these defects.

Methods:

Retrospective analysis of patient records and MRI Images. All MRI studies have been performed on a Brilliance 1T Scanner, Phillips GmbH, Hamburg, Germany. Image sequences consisted of sagittal, transverse and dorsal T2, transversal fluid-attenuated inversion recovery (FLAIR), and transversal T1 precontrast and postcontrast.

Results:

Age at diagnosis ranged from 12 weeks to 7 years. MRI findings were confined to the forebrain in our study. Porencephalic lesions appear as wedge shaped parenchymal defects connecting the ventricular system and the subarachnoid space. One dog with bilateral cystic lesions in the cerebral hemispheres was diagnosed having false porencephaly (pseudoporencephaly). In two dogs, the cavities were pervaded by thin lines of residua brain tissue and the inner lining of the cysts were ill defined. Whereas in two adult dogs, the porencephalic lesions were asymptomatic the other animals showed clinical symptoms depending on the affected cerebral area. Three animals had seizures. Interestingly, four animals showed neurological signs normally not localized to the forebrain (nystagmus, hypermetria). Cerebrospinal fluid examination was unremarkable in all animals.

Conclusions and Clinical Relevance:

The definition of porencephaly as well as its subclassification is not uniform in veterinary medicine. Clinical symptoms of the cerebral defects are variable and not confined to classical forebrain signs. Clinical neurolocalization can differ from the expected localization of the lesions in diagnostic imaging. Although the defects develop intrauterine or postnatal, the clinical symptoms can occur years after birth.
ASSESSMENT OF CORTICAL SULCI FORMATIONS AND DIAGNOSIS OF LISSENCEPHALY IN THREE DOGS USING TRANSCRANIAL ULTRASOUND

T.C.F. Cintra, C.F. Carvalho, J.C. Canola, A.C. Nepomuceno. Faculdade de Ciencias Agrárias e Veterinárias da Universidade Estadual Paulista, Jaboticabal, Brazil

\section{Introduction:}

Lissencephaly is a congenital malformation characterized by total or partial absence of cortical sulcus and gyrus. Clinical signs consist of dementia, aggressiveness, convulsions, visual, and olfactory dysfunctions and circle walking. Magnetic resonance imaging (MRI) can confirm the diagnosis. However, this exam involves high costs such as equipment acquisition and maintenance, therefore making it difficult to perform as a routine, especially in veterinary medicine. Temporal bone has been used as an acoustic window for transcranial ultrasound (TCUS) in humans and dogs. The objectives of this study were to standardize thickness measurements of the marginal and coronal encephalic sulci, visualized by TCUS in dogs, and to describe the ultrasonographic aspects of lissencephaly in three dogs.

Methods:

In order to assessment of the encephalic sulci, it was scanned 30 healthy adult dogs (weighing between 2 -and $10 \mathrm{~kg}$ ), with mesaticephalic cranial conformation. In the experimenta group, TCUS was performed using a convex transducer $(4-7 \mathrm{MHz})$ initially positioned perpendicularly to the temporal bone to obtain a median dorsal plan. Measurement of the marginal sulcus was performed in dorsal oblique plane, with the transducer rotated $45^{\circ}$ in relation to the dorsal plane and with a $30^{\circ}$ angle toward the caudal aspect. Coronal sulcus was measured in a dorsal oblique plane with the transducer rotated $45^{\circ}$ in relation to the dorsal plane with $\mathrm{a} 30^{\circ}$ angle toward the cranial aspect. In order to identify anomalies related to the cerebral sulci, a clinical prospective study was performed in 104 dogs underwent to TCUS between January of 2010 and April of 2011 .

Results:

In the experimental group, both marginal and coronal sulci were visualized as linear hyperechogenic structures within the hypoechoic parenchyma in all 30 dogs and identified according to the anatomy. Thickness of the marginal sulcus ranged from 0.13 to $0.35 \mathrm{~cm}$ (mean of $0.16 \mathrm{~cm}$ ), and thickness of the coronal sulcus ranged from 0.12 to $0.21 \mathrm{~cm}$ (mean of $0.15 \mathrm{~cm}$ ). TCUS clinical study revealed three dogs with absence of sulci images, and one of them also presented dilation of lateral ventricles. They were two Ihasa apso and on poodle, with ages between 18 and 28 months, all of them with behavior disturbances and convulsions. Lissencephaly was confirmed by necropsy in two dogs and by MRI in one. Discussion/Conclusion:

There have been, to our knowledge, no studies directed toward to describing or standardization these sulci using TCUS. Our study demonstrates that marginal and coronal sulci can be visualized in normal dogs through TCUS and this method was used to diagnostic of lissencephaly.

\section{INTRAVENTRICULAR MENINGIOMA ASSOCIATED WITH A CHOLESTEROL GRANULOMA OF THE CHOROID PLEXUS IN A CAT}

N. Ondreka ${ }^{1}$, M. Henrich ${ }^{2}$, M. Kramer ${ }^{1}$, M. Schmidt ${ }^{1} .{ }^{1}$ Klinik fuer Kleintiere - Chirurgie ${ }^{2}$ und Institut fuer Veterinaer-Pathologie der Justus-Liebig-Universitaet Giessen, Giessen, Germany

Case History

A 12-year-old female domestic shorthair cat was presented to the small animal clinic with a history of neurologic signs. Clinical and neurologic examination revealed moderate depression and decreased postural reactions on all limbs. Magnetic resonance imaging (MRI) of the brain was performed at $1 \mathrm{~T}$. MRI revealed a well-defined lobulated space-occupying lesion located in the third ventricle extending to the lateral ventricles. The lesion was of mixed signal intensity with predominantly isointense signal to brain in T2- and T1-weighted spin-echo sequences and fluid-attenuated inversion recovery sequence (FLAIR). Small areas of signal sequences and fluid-attenuated inversion recovery sequence (FLAIR). Small areas of signal
void with irregular multifocal distribution were visible in all sequences. The FLAIR showed void with irregular multifocal distribution were visible in all sequences. The FLAIR showed
a fine hyperintense rim surrounding the lesion. Overall, there was heterogenous contrast a fine hyperintense rim surrounding the lesion. Overall, there was heterogenous contras uptake detectable within the mass after administration of gadolinium on T1-weighted imconsistent with the small regions of signal void in the plain sequences. A mass effect was evident by obliteration of the mesencephalic aqueduct, caudal displacement of the rostral colliculi, intraforaminal displacement of the cerebellum, and dilation of both lateral ventricolliculi, intraforaminal displacement of the cerebellum, and dilation of both lateral ventri-
cles with enlargement of the olfactory recesses emphasized on the right. Based upon the imaging findings the list of differentials included cholesterol granuloma (CG), meningioma ependymoma, and choroid plexus neoplasia with the last ones being less likely. The cat was euthanized and necropsy was performed. Gross examination showed a brownish, firm mass compressing adjacent neuronal tissue by replacement of the choroid plexus of the lateral ventricle and occlusion of the third ventricle. Consecutive to the obliteration of the mesencephalic aqueduct, there was enlargement of the cerebral ventricles and the olfacmesencephalic aqueduct, there was enlargement of the cerebral ventricles and the olfac-
tory recesses. Formalin-fixed samples were processed for histology and stained by routine techniques. Immunohistochemistry was also performed. Histopathology and immunohistochemistry identified the mass as meningioma with a plexus cholesterol granuloma. The mass was composed of numerous microvacuolated macrophages, interspersed giant cells, and numerous acicular clefts adjacent to and intermixed into a proliferation of spindle cells in dense streams and whorls, focally infiltrating into the neuropil. Multifocal hemorrhages and mineralization were present.

Discussion:

CGs are non-neoplastic tumor-like masses characterized by focal aggregation of granulation tissue containing cholesterol crystals or ester. It is hypothesized that these granuloma develop secondary to local tissue changes associated with inflammation or hemorrhage. Intraventricular menigiomas are common in cats, but intracranial CGs are only reported in two cats, both cases arising outside the ventricular system. To our knowledge, this is the first description of an intraventricular CGs in a cat and the first description of the coexistance with a meningioma. The MR features of the CG in this cat are similar to what was reported with a meningioma. The MR features of the CG in this cat are similar to what was reported
in another case report. The areas of signal void appear to be consistent with hemorrhage, calcification, and cholesterole crystals. As meningiomas in cats usually show homogenous signal intensity and homogenous, intensive contrast uptake, this was not on top of the list of our differential diagnoses. 
COMPUTED TOMOGRAPHY, LOW-FIELD MAGNETIC RESONANCE IMAGING AND CROSS-SECTIONAL ANATOMY OF THE HEAD IN HEALTHY RABBITS

S. Borgonovo, D.D. Zani, M. Moioli, S. Gerosa, D. De Zani, S.C. Modina, M. Di Giancamillo. Faculty of Veterinary Medicine, Milano, Italy

\section{Purpose:}

To provide a detailed anatomical description of the head of normal rabbits by using computed tomography (CT) and low-field magnetic resonance imaging (MRI), simulating clinica conditions, and to compare it with a compilation of corresponding gross anatomical sections on transversal, dorsal, and sagittal planes.

Methods:

The heads of five clinically normal New Zealand White rabbits, weighing about $4 \mathrm{~kg}$ and euthanatized for reasons not connected to our study, were submitted to examination with fourth-generation CT (PQ2000S Philips MD S.p.A., Monza, Italy) and low-field MRI (0.18 T, Vet-MR Esaote S.P.A Genova, Italy) before decapitation. In CT study, to obtain a transverse section, each rabbit was positioned in ventral recumbency on CT couch, with its head extended on the neck, whereas to obtain a sagittal section, each rabbit was positioned as above, but with its head flexed at $90^{\circ}$ laterally on the neck. Dorsal images were obtained by placing each rabbit in dorsal recumbency, with its head perpendicular to the couch, and flexed at $90^{\circ}$ ventrally. Three-millimeter-thick slices were acquired on each plane with hard and soft convolution filter. For MRI study, the same rabbits were positioned in ventral recumbency on the MRI couch, with their heads placed inside a volumetric coil. Transverse, sagittal and dorsal 3-mm-thick T1-weighted images, T2-weighted images and Gradient Echo T1-weighted images were performed. CT and MRI transverse and dorsal sections were performed in images were performed. CT and MRI transverse and dorsal sections were performed in the hard palate and to the skull base, respectively, for dorsal ones single slices were obtained parallel to the structure above. Immediately after MRI examination the rabbits were decapitated and their heads were positioned and frozen in an ice cube at $-14{ }^{\circ} \mathrm{C}$ until solid, and then sectioned at 6-mm-thick intervals by using an electric band saw, according to the same section plane applied for CT and MRI scans. Slab sections were immediately cleaned,
photographed, and compared with corresponding CT and MRI images, to identify anatomic structures and match them with the different acquisition. For each cutting plane, we gained a good overlap between the section obtained at the same level with different techniques (CT, MRI, and cross sectional anatomy).

Results:

The bone-window CT images provided a good anatomic detail of dentition and hard structures of rabbit skulls, and the soft-tissue structures were well valuated with the soft-tissue window. However, T1- and T2-weighted images provided detailed information regarding sof tissue and central nervous system structures, and Gradient Echo T1-weighted acquisition supplied adequate information about bone and teeth.

Conclusions:

The results of this study could be used as a guide for evaluating CT and MRI images of rabbits with head disorders. It is important to highlight that rabbits positioning both in CT and MRI studies were reproducible with anesthetized subjects, for clinical investigation also on dorsal and sagittal plane of acquisition.

\section{DIAGNOSTIC IMAGING FEATURES OF CANINE INFRAORBITAL CYSTS}

T. Schwarz ${ }^{1}$, S. Pot ${ }^{2}$, E. Bentley ${ }^{3}$, A.I.C. Marques ${ }^{1}$, S. Smith ${ }^{1}$, R. Drees ${ }^{3} .{ }^{1}$ The Royal (Dick) School of Veterinary Studies, Roslin, Scotland UK, ${ }^{2}$ Vetsuisse Faculty, University of Zurich, Zurich, Switzerland, ${ }^{3}$ University of Wisconsin-Madison, Madison, WI

\section{Introduction:}

Benign cystic lesions arising from the nasolacrimal duct (dacryops) or lacrimal canaliculi (canaliculops) are a rare disorder in dogs that can be treated surgically. Purpose of this study was to describe the radiologic, computed tomographic (CT), and magnetic resonance imaging (MRI) features of this condition.

Methods:

Medical and histopathological records were searched for confirmed cases of cystic infraorbital cystic lesions with supportive imaging studies, including survey and contrast radiography, CT, and MRI. Imaging features in all modalities were reviewed and compared. phy, CT, and

Seven dogs were included in the study. All lesions were expansile, fluid-filled, thin-walled infraorbital cysts along the path of the nasolacrimal duct often invading the maxillary recess and nasal cavity. Radiographically the lesions appeared as well-circumscribed infraorbita radiolucencies. On contrast radiography, lesions did not connect with the nasolacrimal or salivary glands or ducts. Evaluation of CT and MRI images revealed local bone atrophy, contrast enhancing cyst walls and nonenhancing fluid content, and absence of any aggressive lesion characteristics or lymphadenopathy. CT-dacryocystography did not reveal a cyst sive lesion characteristics or lymphadenopathy. CT-dacryocystography did not reveal a cyst
connection with the nasolacrimal duct. The tissue of origin was variable on histopathological connection with the nasolacrimal duct. The tissue of origin was variable on histor
examination (nasolacrimal duct, respiratory epithelium, and salivary tissue). examination (nas

Cystic lesions in the infraorbital region have characteristic radiographic, CT, and MRI features and should be recognized as a benign condition and differentiated from malignant infraorbital lesions in the dog. In the veterinary literature, these lesions have been described as dacryops or canaliculops whereas in fact they do not necessarily originate from the lacrimal system and may have different etiologies. Histopathological confirmation is recommended if these lesions are surgically removed.

\section{ULTRASONOGRAPHIC EVALUATION OF THE THYROID GLAND IN HEALTHY DOGS} OF DIFFERENT AGE GROUPS

T.R. Muller, M. Assis, D.P. Doiche, R. Sartor, M.J. Mamprim. School of Veterinary Medicine and Animal Science, São Paulo State University, Botucatu, Brazil

\section{Introduction:}

The thyroid gland was assessed by ultrasound in healthy medium-sized dogs in different age groups. The objective was to determine patterns of image in these groups, as well as identify possible changes in image resulting from aging.

Methods:

Thirty dogs-10 young, 10 adult, and 10 elderly - without clinical signs or history of thyroid disease, with $\mathrm{CBC}$ and thyroid function tests (total $\mathrm{T}_{4}$ and $\mathrm{TSH}$ ) within the reference values were evaluated. Each thyroid lobe was examined by ultrasound for shape, size, echogenicity, and echotexture. The analysis of echogenicity and echotexture was made by histogram. Thyroid volume was estimated by the equation for ellipsoid (length $\times$ width $\times$ height) $\pi / 6$. Results:

The results indicate that the thyroid volume of young dogs has a tendency to be higher than in adult dogs $(P=0.068)$ and older dogs $(P=0.120)$. The height of thyroid lobe in the longitudinal plane was significantly higher in young dogs compared with the other dogs. The echotexture and echogenicity had no significant difference between groups, but the echogenicity was greater in older dogs.
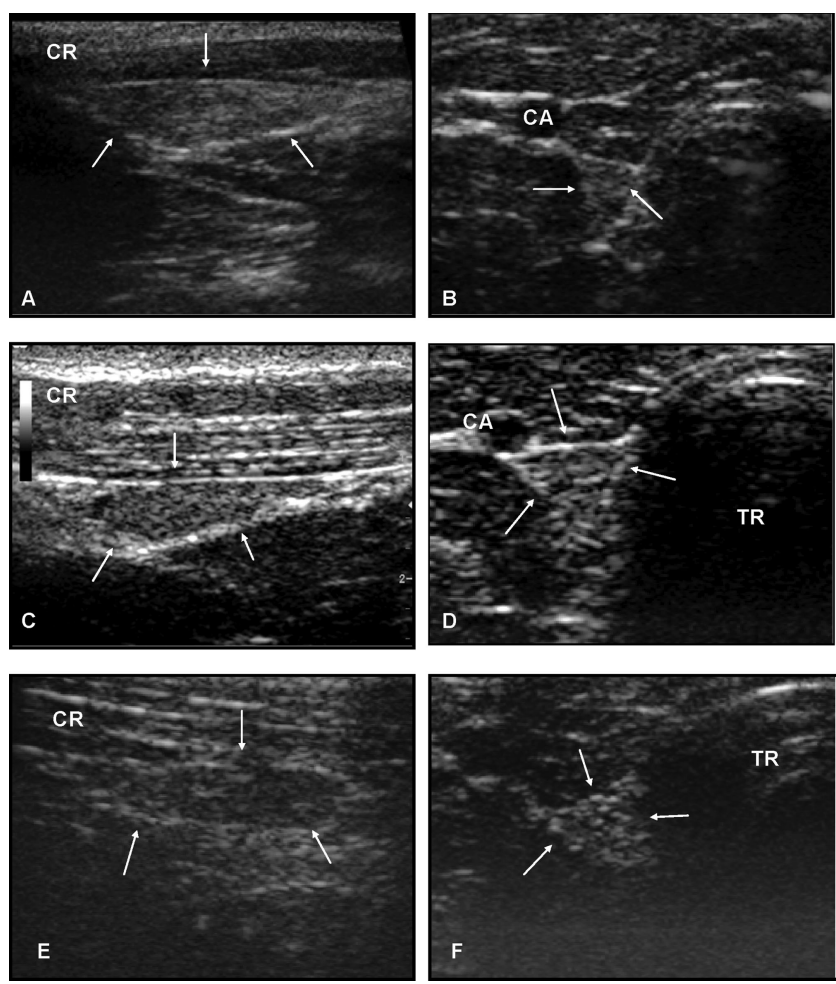

FIG. 1. Sonograms in the longitudinal plane $(A, C$, and $E)$ and transverse $(B, D$, and $F)$ of the thyroid lobes in normal young dogs ( $A$ and $B$ ), adults dogs ( $C$ and $D)$, and old dogs $(\mathrm{E}$ and $\mathrm{F})$. The margins of the thyroid lobes are marked with white arrows. CR, cranial; $\mathrm{CA}$ carotid artery; TR, trachea.

Conclusion:

The results indicate that ultrasound imaging of the thyroid is influenced by age in dogs of medium size. 


\section{CT AND MRI FINDINGS OF CERVICAL STENOSIS CAUSED BY MALFORMATION OF THE ARTICULAR FACETS AND MENINGEAL FIBROSIS OF C2-C3 IN TWO YOUNG LARGE BREED DOGS}

K. Kromhout ${ }^{1}$, I. Gielen ${ }^{1}$, L. Van Ham ${ }^{2}$, H. van Bree ${ }^{1} .{ }^{1}$ Department of Veterinary Medica Imaging and Small Animal Orthopaedics, ${ }^{2}$ Small Animal Medicine, Faculty of Veterinary Medicine, Ghent University, Belgium

\section{Introduction/Purpose:}

Cervical stenosis with spinal cord compression in large breed dogs is a common disorde and is described in numerous articles. Causes are variable but are linked to the cervica vertebral malformation-malarticulation syndrome or cervical spondylomyelopathy. Affected vertebrae range from $\mathrm{C} 3-\mathrm{C} 4$ till $\mathrm{C} 6-\mathrm{C} 7$. To our knowledge, this is the first report to describe the CT and MRI findings of malformation of the articular facets of $\mathrm{C} 2-\mathrm{C} 3$ and meningeal fibrosis as a cause for cervical stenosis in two young large breed dogs.

Materials and Methods:

A 13-month-old Labrador retriever and a 6-month-old St. Bernard were presented at th University Clinic of Small Animals with front and rear limb ataxia. Both patients underwentin their clinical work-up-both MRI and CT studies. MR images were obtained on a 0.2-T unit (Hitachi ${ }^{8}$ Airis Mate). The animals were positioned in dorsal recumbency under general anesthesia with their neck placed into a human neck coil and submitted to T1- and T2 weighted spin-echo, FLAIR, STIR, and postgadolinium T1-weighted sequences in severa planes. CT transverse images were obtained with a helical multislice CT scanner (GE Lightspeed QX/I).

Results:

MR-images of both dogs showed a bilateral dorsolateral compression of the spinal cord a the level of the articular facets of $\mathrm{C} 2-\mathrm{C} 3$. On the T1- and T2-weighted, STIR and FLAIR images at the level of the articulation of $\mathrm{C} 2-\mathrm{C} 3$, a focal extramedullary hyperintense welldefined mass was detected dorsal from the spinal cord. A dorsal connection between the dura and the mass was visible. Dorsally from the processus spinosus of C3 a well-defined dura and the mass was visible. Dorsally from the processus spinosus of $C 3$ a well-defined um nuchae. On the postcontrast images, a homogeneous contrast uptake was visible in the dorsal extramedullary mass. On CT images, an asymmetric malformation was visible in the cranial articular facets of C3. A dorsal extramedullary hypodense mass was visible which caused compression on the spinal cord. Normal outlining of the dorsal epidural space was absent. A hypodense mass was present dorsal of the processus spinosus in the region of the ligamentum nuchae. Both dogs underwent a dorsal laminectomy on the level of $\mathrm{C} 2-\mathrm{C} 3$. On inspection, a fibrous mass was visible between the laminae of $\mathrm{C} 2$ and $\mathrm{C} 3$. The mass connected to the ligamentum flavum and the meninges. Histology of the mass of one dog showed it contained fat and connective tissue combined with mineralizations.

\section{Discusssion/Conclusions:}

In young dogs cervical dorsolateral stenosis can be caused by osseus congenital malformations or acquired processes such as hypertrophied ligaments, degenerative articula changes, and synovial cysts. Dorsal extramedullary compression in young dogs can be the result of meningeal fibrosis. This kind of cervical fibrotic stenosis is seen in Rottweilers, Bernese mountain dogs, and Labrador retrievers. CT and MRI are of great value to determine the cause of these forms of stenosis.

\section{SONOGRAPHIC PARAMETERS FOR ASSESSMENT OF CERVICAL VESSEL INVASION BY TUMORS}

C.F. Carvalho; C.R. Novelli; C.M. Simão. Universidade Cruzeiro do Sul, São Paulo, Brazil.

\section{Introduction/Purpose:}

Tumor invasion of the vessels displays both therapeutic and prognostic implications and represents a challenge to head and neck surgeons. Although previous research has shown that ultrasound can detect such invasions, accurate sonographic parameters to do so have not yet been established. The objective of our study is to evaluate previously proposed criteria for cervical carotid artery and internal jugular invasion through ultrasound (US) in order to set the most accurate parameters for cervical vessels sonographically in dogs. Methods:

A high-resolution transducer was used to perform US examinations (B-mode, color, and pulsed Doppler) of 15 patients, selected from a group with inconclusive radiography and computed tomography (CT) diagnosis. The criteria evaluated through B-mode US were (1) loss of vessel wall of bright hyperechogenic layer in the contact area with tumor; (2) reduction of vessel caliber and lumen deformity; (3) vascular encasement based on contact area between tumor and vessel up to $180^{\circ}$ (thrombosis and/or tumor protrusion into the lumen); (4) immobility evaluating tumor adherence to the vessels characterized by dynamic maneuvers, obtained with transducer in the suspected area (transversal section) waiting for swallowing and doing a light pressure with the transducer over the region. Visualization of vessel invasion was performed during surgery and was considered gold standard.

Results:

In order to define the best parameters, we evaluated sensitivity and specificity for each criterion. We found that tumor immobility or fixation in the vessel wall, encasement, and reduction of vessel caliber and/or deformity of the lumen were the best criteria. Indeed, when loss of hyperechoic interface of the vessel wall was used as a single criterion it generated false positive results. The three parameters together, improved sensitivity, specificity, and the positive and negative predictive values obtained.

Discussion/Conclusions:

To set the value of sonographic criteria for detection of vessel invasion by tumors, we evaluated four parameters in patients with doubtful vascular invasion diagnosis from either physical, radiographic, and/or CT exams. This study showed that a combination of parameters could be used to provide the best sensitivity and specificity values to produce conclusive diagnosis of vessel invasion by tumors in the cervical region.

\section{A COMPARISON OF TWO TECHNIQUES FOR ULTRASOUND-GUIDED BRACHIAL PLEXUS BLOCK IN CATS}

A. Anson ${ }^{1}$, F. Gil ${ }^{2}$, F.G. Laredo ${ }^{1}$, M. Soler ${ }^{1}$, M.D. Ayala ${ }^{2}$, E. Belda ${ }^{1}$, A. Agut ${ }^{1} .{ }^{1}$ Department of Animal Medicine and Surgery, Veterinary Teaching Hospital, ${ }^{2}$ Department of Anatomy and Compared Anatomy, University of Murcia, Spain

\section{Introduction/Purpose:}

Regional anesthesia (RA) has traditionally been performed using anatomical landmarks or peripheral nerve (PN) electrostimulation to locate the nerves. These "blind" techniques have a significantly high failure rate and are potentially associated to complications. In recent years, ultrasound (US)-guided techniques are gaining popularity in RA to facilitate PN blocks and have been successfully employed in dogs. To the author's knowledge, there is little information regarding the use of these techniques in cats. The aim of this study is to describe the anatomical basis and the US approaches to block the brachial plexus in the to de Methods:

Ten fresh adult feline cadavers were used. The forelimbs were scanned at the axillary area using an 18-MHz linear transducer. The brachial plexus block was approached by US in two different procedures. In the first procedure, the cats were positioned in dorsal recumbency with both thoracic limbs extended caudally and the block was performed in the right brachial plexus. In the second procedure, the cats were placed in the same position, but the left forelimb to be blocked was abducted in $90^{\circ}$ and the other one extended caudally. In both forelimb to be blocked was abducted in $90^{\circ}$ and the other one extended caudally. In both
cases, the transducer was orientated in a parasagittal plane on the axillary area and it was tilted until the brachial plexus was visualized on a transverse plane. Then, the needle was positioned in plane and the structures identified as components of the brachial plexus were injected with $0.3 \mathrm{ml} / \mathrm{kg}$ of blue ink. The insertion sites of the needle into the skin as well as the anatomical landmarks employed were marked and photographed to serve as reference points to describe the most optimal acoustic window to approach the plexus. Ten of the limbs were dissected immediately to confirm the presence of blue ink staining the target nerves. Transverse cryosections were made in the others 10 limbs to demonstrate the existence of blue ink around the nerves.

Results:

For both approaches, the anatomical landmarks employed were the acromion of the scapula the scapulo-humeral joint and the first rib. The brachial plexus could be identified clearly by US in all cases. The axillary approach, in which the limb to be blocked, was abducted $90^{\circ}$ was easier to be performed. In $7 / 10$ forelimbs, all the nerves of the plexus were successfully stained. In $2 / 10$ forelimbs, the suprascapularis and subscapularis nerves were not stained. In $1 / 10$ forelimbs, the jugular vein was punctured and the presence of blood obscured the ink staining in the axillary space. The three cases, where the brachial plexus was not stained correctly, corresponded to the approach in which the forelimbs were extended caudally. The cryosections confirmed the presence of blue ink around of all the components of the brachial plexus in all cases.

Conclusions:

The axillary approach in which the forelimb to be blocked is abducted $90^{\circ}$ and the other is extended caudally provided the most effective approach to perform an US-guided block of the brachial plexus in the cat. 
VALUE OF ULTRASONOGRAPHY IN DETECTING CAPSULAR THICKENING IN DOG WITH ELBOW SYNOVIAL OSTEOCHONDROMATOSIS

K. Szczepanska ${ }^{1}$, M. Lobacz ${ }^{2}$, W. Atamaniuk ${ }^{3}$, J. Lorenc ${ }^{1} .{ }^{1}$ Private Practice, Poland ${ }^{2}$ Faculty of Veterinary Medicine, University of Glasgow, Scotland, ${ }^{3}$ Faculty of Veterinary Medicine, Wroclaw University of Environmental and Life Sciences, Poland

\section{Introduction/Purpose:}

Osteochondromatosis is a rare disease in dogs. The thickness of soft tissues can be measured on MRI and mineralization is better detected by radiography, while histopathology is required for a final diagnosis. Combination of radiographic and histopathological examination is required for a final diagnosis. Ultrasonography can be an ideal imaging technique to assess the capsule because it is noninvasive, more available, less expensive than MRI or CT technique, and does not require sedation or general anesthesia. The purpose of this report was to present the ultrasonographic appearance of ossified bodies in osteochondromatosis. was to present the ultrason

Materials and Methods: formed using a 10-14 MHz linear transducer (Logiq pq5). The appearance and thickness of the capsules was evaluated twice 4 months apart. Histopathologic examination, radiographs, and CT examination was also performed before surgery.

Results:

During the first US examination severe elbow swelling and multiple cartilaginous lesion were found. Ultrasonographic appearance of synovial osteochondromatosis: small volume of mildly echogenic fluid was detected. The near surface of the mineralized bodies had curved appearance with irregular, hyperechoic margins, causing distinct acoustic shadowing (curvilinear area, Fig. 1A). Considerable anechoic fluid was found between the bodies. A distinct hypoechoic capsule $(0.2-0.3 \mathrm{~cm}$ thick) was found in three different regions with no surrounding fluid. Four months later, there was larger amount of mineralized bodies and capsule thickness remained the same. Gross and histopathological appearance of the capsule was comparable with the ultrasound findings (Fig. 1A and B).
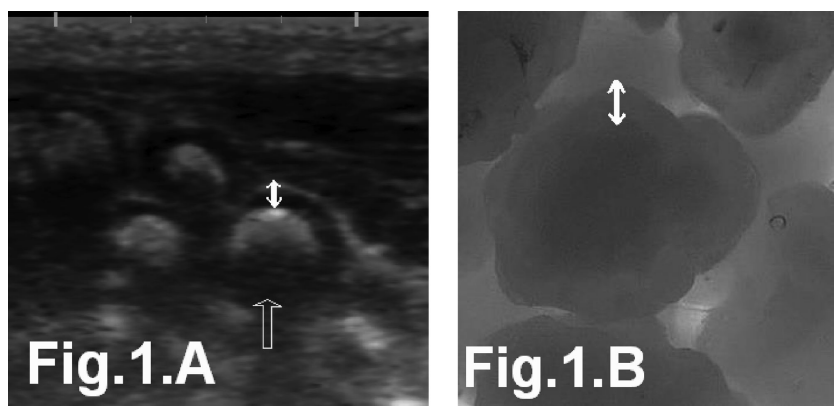

FIG. 1. Capsular appearance. (A) Ultrasound image of capsular thickness measurement (white arrow), acoustic shadow artifact (open arrow). (B) Gross view of capsular thickness measurement (white arrow).

Conclusion:

Ultrasonography is noninvasive and valuable method to determine pathological features such as capsule thickening in osteochondromatosis located close to the skin surface. Further investigation is needed to establish the range of normal thickness in benign and malignan ostochondromatosis in dogs.

\section{ANATOMIC AND ULTRASONOGRAPHIC STUDY OF THE CANINE ELBOW}

A. Villamonte Chevalier ${ }^{1}$, R. Latorre ${ }^{2}$, M. Soler ${ }^{1}$, F. Gil $^{2}$, R. Sarria ${ }^{2}$, A. Agut ${ }^{1} .{ }^{1}$ Department of Medicine and Surgery, ${ }^{2}$ Department of Anatomy and Compared Pathological Anatomy, University of Murcia, Spain

\section{Introduction/Purpose:}

Ultrasonographic study of the canine elbow joint has been previously reported. High frequency transducers provide more detailed images of structures evaluated. The purpose of this study was to assess the canine elbow joint using a high-frequency transducer $(18 \mathrm{MHz})$ describing and correlating the ultrasonographic images with anatomical sections performed on the same planes used in the ultrasonographic evaluation.

Methods:

Anatomical study: The dissection of the elbow joint was carried out in 10 forelimbs obtained from five German Shepherd-crossed breed dog cadavers. Transparent sections (2-mm thick) were obtained from elbow joints at $-70^{\circ} \mathrm{C}$, sections were preserved using the $\mathrm{E} 12$ plastination technique. Ultrasonographic "in vitro" study: the elbow joint was ultrasonographically nation technique. Ultrasonographic "in vitro" study: the elbow joint was ultrasonographically
evaluated using an 18-MHz linear array transducer in 10 elbow joints from five fresh cadavers
of German Shepherd-crossed breed dogs euthanized for unrelated reasons to orthopedic pathologies. A protocol of the ultrasonographic windows on the transversal, longitudinal, and oblique planes was established approaching the medial, caudal, and lateral aspects of the elbow joint. Correlations between ultrasonographic and anatomic images were made. Results:

The anatomical dissection of the elbow joint allowed establishing the anatomical landmarks to be employed to locate the structures on ultrasound evaluation. On the medial aspect, the anatomical landmarks used were the biceps brachii muscle, and the humeral epicondyle. The insertion tendons of brachialis and biceps braquii muscles were assessed, as well as the medial collateral ligament and the medial coronoid process. On the caudal aspect, the anatomical landmarks were the olecranon tuberosities and the humeral condyle. In this approach, the tendon of insertion of the triceps brachii muscle, the anconeal process and the olecranon ligament were assessed. On the lateral aspect, the anatomical landmark used was the humeral epicondyle. The lateral collateral ligament, anconeal muscle, and lateral was the humeral epicondyle. The lateral collateral ligament, anconeal muscle, and lateral coronoid process were evaluated. The ultrasonographic appearance of the muscles was as
hypoechoic structures with hyperechoic lines within. The ligaments were dotted hyperechoic or hypoechoic structures depending on the angle of the ultrasound beam. Bone structures were observed as hyperechoic lines with acoustic shadowing. There was a good correlation between the ultrasonographic images and anatomical sections.

Discussion/Conclusion:

Our results agree with those obtained in previous studies; however, in this study a better resolution of soft-tissue structures such as muscles, ligaments, and tendinous structures was observed. In particular, on the medial aspect, the attachment of the tendons of biceps brachii and brachialis muscles on the ulnar tuberosity was described in this study, which has not been reported in previous publications.

\section{DEVELOPING A TECHNIQUE FOR CT-GUIDED LUMBAR PERIRADICULAR INJECTION-A CADAVER STUDY}

F. Willmitzer, M. Leschnik, S. Breit, S. Dengg, J. Thalhammer, A. Pakozdy, S. Kneissl. De partment of Small Animals and Horses (Willmitzer, Leschnik, Dengg, Thalhammer, Pakozdy, Kneissl) and Department of Pathobiology (Breit), University of Veterinary Medicine, Vienna, Austria

\section{Introduction/Purpose:}

European guidelines for the management of chronic nonspecific lower back pain provided a favorable level of evidence for transforaminal epidural steroid injections in man. To develop a technique for CT-guided periradicular infiltration (synonymous with transforaminal or selective nerve root injections) in dogs and define the success rate, a cadaver study has been

performed.

Methods: nized for unrelated reasons, CT-guided periradicular infiltration of L1-L5 was performed using a mixture of methylene blue $(2.9 \mathrm{ml})$ and iodinated contrast medium $(0.1 \mathrm{ml} \mathrm{Scanlux}$ Sanochemia). The goal of the intervention was to position the tip of the needle at the level of the cranial aspect of the intervertebral foramen, as close as possible to the ganglion of the dorsal nerve root, and to control the injection site by CT. Aspiration was performed prior to the injection to exclude intravascular position. Then the cadaver was frozen and anatomic the injection to exclude intravascular position. Then the cadaver was frozen and anatomir sections were cut in the same plane as the cross-sectional CT images were acquired. Fur-
thermore, a detailed anatomic dissection was performed and verified for methylene blue dying of neural structures macroscopically and mesoscopically (using a magnifying glass). Results:

Successful application - as close as reasonable to the ganglion of the dorsal nerve rootwas achieved in $5 / 10$ sites with needle placement at the cranial aspect of the intervertebral foramen and at the dorsal contour of the vertebral body. Noteworthy the deep leaf of the thoracolumbar fascia and the tendon of the iliocostal muscle initially formed a clear demarcation line for methylene blue, if not perforated by the tip of the needle. The spinal nerve (i.e., ventral branch of spinal nerve) was colored in $7 / 10$ sites, the result was questionable in $3 / 10$ sites. Compared to CT, the injected mixture resulted in about a distinctly large area within the epaxial musculature on the frozen sections. Contrast medium was located circumferentially to the tip of the needle.

\section{Discussion/Conclusions:}

Lower back pain is a common cause of debilitation in large breed dogs. Periradicular infiltration can be used as a diagnostic test to confirm the level of the pathology in radicular pain or pain therapy. There was a learning curve with faster and more precise application with time, using CT. It is hypothesized that the difference in volume and distribution between contrast medium and methylene blue resulted from postinterventional changes or relatively low contrast concentration. There is no risk for subarachnoid penetration, if the tip of the needle is placed at the cranial aspect of the intervertebral foramen. Further cadaver studies are needed to improve the accuracy and repeatability of this technique.

Reference:

1. Airaksinen O, Brox JI, Cedraschi C et al. Chapter 4: European guidelines for the management of chronic nonspecific low back pain. Eur Spine J 2006; 15:S192-S300. 
VALUE OF ULTRASONOGRAPHY IN THE DIAGNOSIS OF LUNG LOBE TORSION IN DOGS: COMPARISON BETWEEN ULTRASONOGRAPHIC, RADIOGRAPHIC, AND CT IMAGES IN TWO CLINICAL CASES

G. Gory ${ }^{1}$, E. Cauvin ${ }^{1}$, H. Gallois-Bride ${ }^{2}$, D.N. Rault ${ }^{1} .{ }^{1}$ AZURVET, Referral Center in Veterinary Diagnostic Imaging and Neurology, 06800 Cagnes-sur-mer, ${ }^{2}$ OLLIOLIS, Referra Center in Veterinary Surgery, Dentistry, Internal Medicine and Diagnostic Imaging, 83190 Ollioules, France

\section{Case Histories:}

A 5-year-old female Pekingese dog was presented for acute lethargy, weakness, dyspnea and increased respiratory efforts of 2-day duration. Examination showed severe bradycardia. This dog was referred for ultrasonographic thoracic examination, which revealed the presence of moderate anechogenic pleural effusion. Cardiac examination was unremarkable and no sign of pulmonary hypertension was noticed. The left lung surfaces appeared smooth, regular, and partially collapsed. On the right side of the chest, dorsocaudal to the heart, a large, convex, and irregular surface was observed. It was characterized by granular, hyperechoic interface, with speckled reverberation artifacts. This surface could be followed over a whole lung lobe, with hypoechoic areas at the periphery and ventrally, where discrete hyperechoic areas suggested entrapped gas. Doppler examination failed to revea any blood flow in the echogenic consolidated portions of the affected lung. Radiographic and CT examinations were subsequently performed. X-rays showed an enlarged right lung lobe with a vesicular appearance. CT confirmed right middle lobe torsion.

An 8-year-old, spayed female Beauceron was presented with a 1-day history of dyspnea, and 5-day history of dry cough and lethargy, not responding to steroid therapy. This do was referred because of suspicion of lung lobe torsion (LLT) on radiographic examination. A mixed, vesicular alveolar pattern was noticed over the left cranial lung lobe projection, associated with moderate pleural effusion. Thoracic ultrasonographic examination revealed, dorsocranially on the left, homogeneous, consolidated portions of lung containing visible anechoic tubular images totally devoid of blood flow. The surface of the affected lobe could be followed to an irregular, rough, and granular interface. The periphery of the lobe was hypoechoic and heterogeneous, deeper areas contained granular and pouch-like hyperechoic areas. CT confirmed torsion of the left cranial lung lobe.

Discussion:

These two cases illustrate the value of ultrasound to investigate LLT in dogs. CT remains the gold standard to confirm and define LLT. However, ultrasonographic images in these two cases were highly suggestive of this condition in agreement with the CT appearance. The vesicular pattern described for radiography and CT led to a typical granular and speckled appearance on ultrasound. The affected lobe was enlarged, bag-like, and partially air filled. Comparison of CT and ultrasonographic images allows us to understand that the surface of the entire lobe is altered because of entrapped air bubbles and a mixed alveolar pattern corresponding to necrosis, hemorrhage and edema. The association of granular surface,

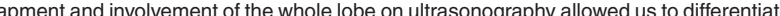
torsion from pneumonia, atelectasis, thromboembolism, contusion, tumor, hemorrhage, or abscess. Furthermore, pleural effusion is better ascertained on ultrasonography than on X-rays. Ultrasonography therefore appears to be a valuable tool to confirm a radiographic or clinical suspicion of LLT.

\section{RADIOGRAPHIC APPEARANCE OF BROMIDE-ASSOCIATED BRONCHITIS IN SEVEN CATS}

C. Bertolani, A. Gabriel, J. Hernandez, E. Gomes. CHV Frégis, Arcueil, France

Introduction:

Potassium bromide $(\mathrm{KBr})$ is an anticonvulsivant drug extensively used in human and dogs. In cats, its effect on controlling seizures has also been demonstrated. Nevertheless, an association between $\mathrm{KBr}$ treatment and respiratory clinical signs has been described. The association between $\mathrm{KBr}$ treatment and respiratory clinical signs has been described. The associated bronchitis.

\section{Materials and Methods:}

Diagnosis of bromide-associated bronchitis had been made based on the development of lower respiratory signs, including cough and dyspnea while being on bromide therapy and improvement of the condition after drug withdrawal. For each case, available work-up was collected and also used in the diagnosis: signalment, history, time interval between the beginning of $\mathrm{KBr}$ therapy and first respiratory signs, treatment prior to referral, clinical signs; hematologic, biochemical, radiographic, bronchoscopic, broncho-alveolar lavage (BAL), and histopathologic findings, treatment, response to treatment, and follow-up.

Results:

Seven cats were diagnosed with bromide-associated bronchitis. Age at presentation ranged from 1 year to 8 years. Breeds represented were Domestic Shorthair (5), Domestic Longhair (1), and Chartreux (1). All cats had received or were still on $\mathrm{KBr}$ except one cat that received $\mathrm{NaBr}$ for various neurologic diseases. Clinical signs most frequently observed include cough $(7 / 7)$ and dyspnea (2/7). On physical examination, severe restrictive dyspnea (1/7), moderate expiratory dyspnea (1/7), tachypnea (2/7) increased respiratory sounds (3/7), and wheezes (1/7) were observed. Treatments given before presentation included antibiotics in four cats, steroids in five cats, and butopiprine in two cats, no significant improvement was seen. Thoracic radiographs showed moderate to severe generalized bronchial pattern with peribronchial cuffing $(6 / 7)$, micronodular pattern (1/7), multiple nodular lesions (1/7) atelectasis of various lung lobes in (4/7), hyperinflated lung fields $(3 / 7)$, patchy pulmonary
mineralization, and bronchiectasis (1/7). Bronchoscopy and BAL were realized in two cases. BAL analysis showed inflammatory cells (neutrophils [2/7] and macrophage [2/7]) and infectious agents in two cases (E. coli [1/7], P. multocida [1/7], P. mirabillis [1/7], and Candida spp. [1/7]). All cats were discharged on prednisolone and bromide was discontinued in al cats and replaced by phenobarbital in all but one case. Some cats were also treated with inhaled fluticasone (3/7) and/or antibiotics (3/7). All cats improved initially, but one of them developed severe restrictive dyspnea secondary to pneumothorax. Thoracoscopy revealed multiple nodular lesions in all pulmonary lobes. Histopathology revealed an endogenous lipidic pneumonia. In all other cases, clinical and radiological improvement was noted: antibiotic therapy was stopped in all cats and steroid dosage was progressively decreased. Radiographic improvement was noted in four cats but a residual bronchial and peribronchia pattern persisted in some cases. Pulmonary mineralization and atelectasis decreased but persist in some cats, even after improvement of clinical signs. No radiographic control was realized in two cases.

Discussion and Conclusion:

Although it is not possible to establish a direct causality between $\mathrm{KBr}$ administration and the respiratory signs described in these cats, the reversibility of clinical and radiographic signs and the interruption of treatment lean to drug-induced bronchitis.

\section{RADIOGRAPHIC, ULTRASONOGRAPHIC, AND CT FINDINGS OF CANINE} PERICARDIOPERITONEAL DIAPHRAGMATIC HERNIA

K.M. Zardo, M.J. Mamprim, L.C. Vulcano, R. Sartor, V.R. Babicsak, D.R. dos Santos, P.C Jark, J.C. Oshika, R. Camargo, L.H.A. Machado, P.M. de Souza. School of Veterinary Medicine and Animal Science, São Paulo State University, Botucatu, Brazi

Peritoneopercardial diaphragmatic hernia (PPDH) is a rare congenital anomaly mostly found in Weimaraner dogs and Persian cats. Affected animals may be either asymptomatic or show respiratory and gastrointestinal signs. This study aims to describe the radiographic, ultrasonographic (US), and computed tomographic (CT) findings in two cases of PPDH in female mongrels: one, asymptomatic, approximately 3 years of age (case 1); the other, symptomatic with dyspnea, lethargy and appetite loss, 6 years of age (case 2). The radiographic exam nation of the animals' thorax showed dorsal deviation of the trachea, enlargement of cardiac silhouette with heterogeneous soft-tissue radiopacity (cases 1 and 2) and fat and air (case 2), and loss of definition of the ventral diaphragm. Ultrasonography showed the abdominal organs displaced in the pericardial sac: spleen (cases 1 and 2), part of the liver and intraheporgans displaced in the pericardial sac: spleen (cases 1 and 2), part of the liver and intrahep-
atic arteriovenous fistula, intestinal loop, and part of the stomach (case 1). The radiographic atic arteriovenous fistula, intestinal loop, and part of the stomach (case 1). The radiographic radiopacities. Besides that, the presence of gas in gastrointestinal tract adjacent to the heart can impair the ultrasonographic evaluation. Owing to that, the two cases were submitted to CT scan, which showed an aperture in the diaphragm measuring about $7.0 \mathrm{~cm}$ thick by 9.0 $\mathrm{cm}$ wide. The spleen was fully inserted into the pericardial sac in both cases, despite being an organ uncommonly cited in previous studies of PPDH in small animals. Liver, stomach omentum, and intestine, most commonly reported, were partially identified in the pericardial cavity in case 1 (Figure 1)

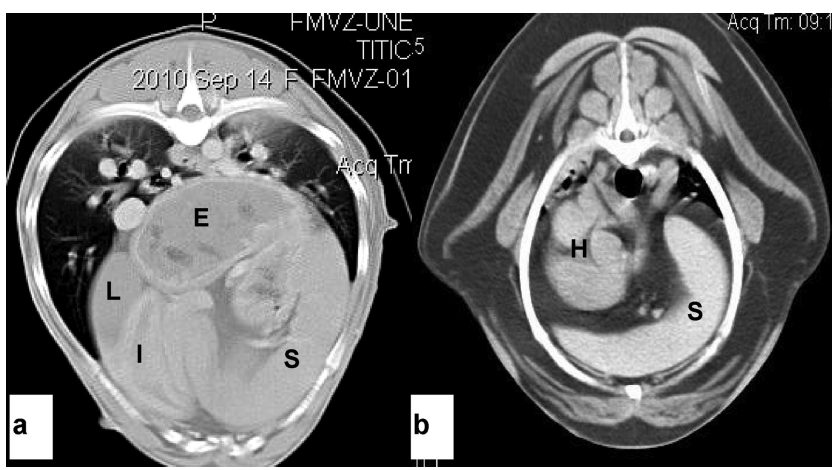

FIG. 1. Tomographic image (5-mm thick) at ninth intercostal space (a: case 1) and the fourth intercostal space (b: case 2). S, spleen; H, heart, E, stomach; L, liver; I, intestine.

Reports of intrahepatic arteriovenous fistula in dogs with PPDH were not found for this study, but it is known that vascular malformations are common in these animals. CT is not affected by the presence of gases in structures around the heart, which allows a complete evaluation and a definitive diagnosis. We concluded that imaging techniques are useful tools in diagnosing PPDH, regarding identification of the herniated structures, their placement and size of the aperture; relevant data supply to establish the prognosis and planning of surgical correction. 


\section{IATROGENIC PNEUMOPERICARDIUM A DOG: CASE REPORT}

W. Atamaniuk, J. Sowa, J. Ostrowska, W. Kinda. Wroclaw University of Environmental and Life Sciences Faculty of Veterinary Medicine, Poland

\section{Introduction:}

Pneumopericardium is a rare condition. Etiology can be divided into three broad categories: trauma, surgery, and fistulation between pericardium and a hollow or air-containing structure, for example, pleural space, pulmonary tissue, bronchial tree, gastrointestinal tract. Much less common pneumopericardium is secondary to gas production de novo by microorganisms invading the pericardial sac, for example, Clostridium perfringens and Klebsiella.

\section{Methods:}

A 10-year-old Boxer, intact male with the history of progressive ascites and hydropericardium was treated in a private practice for 2 weeks. Complete blood tests and serum chemistry profile were normal. Laboratory tests of the fluid aspirated from the pericardial sac revealed the presence of the leukocytes $(0.54 \mathrm{G} / \mathrm{l}$, lymphocytes $90 \%$, neutrophils $10 \%$ ) and erythrocytes $(0.49 \mathrm{G} / \mathrm{l})$. After 2 weeks, there was no response for treatment. About $75 \mathrm{ml}$ of fluid was again aspirated from the pericardial sac and $500 \mathrm{ml}$ from peritoneal cavity.

\section{Results:}

Dog was referred to the Small Animal Clinic, Faculty of Veterinary Medicine in Wroclaw one day after pericardiocentesis. The sound of "splashing mill wheel" was audible during clinica examination. Blood tests revealed decreased amount of lymphocytes in the blood profile. On radiographs, there was a marked (approximately $50-100 \mathrm{ml}$ ) amount of gas present in the pericardial sac, surrounding heart muscle. Ultrasound examination was also performed in this case. It revealed presence of gas in the pericardial sac as typical "comet tail" artifacts, although approach to the air-filled part of the sac was limited. Overall condition of the dog was satisfactory, that is why any surgical intervention was not undertaken. Dog was observed for one week with conservative treatment. Repeated radiographs and ultrasound did not revea any trace of pneumopericardium.

\section{Discussion:}

In the presented case, the cause of pneumopericardium was not obvious. The most probable was iatrogenic cause, but it is hardly to believe that pneumopericardium was a direc aftermath of the pericardiocentesis. The most probable cause of pneumopericardium in this case was a side effect of hyperventilation during anesthesia as it was described in neonate and adult humans. ${ }^{1,2}$ Other causes of pneumopericardium include unintentional damage of the lung during needle placement or other type of communication between pericardia space and lungs. ${ }^{3}$ Blunt thoracic trauma, the most common cause of pneumopericardium in humans, cannot be excluded.

References:

1. Neal RC, Beck DE, Smith VC, Null DM., Neonatal pneumopericardium with high frequency ventilation. Ann Thorac Surg 1989;47:274-277.

2. Neal MD, Hackam DJ. Tension pneumopericardium in an infant. Surgery 2011:149:457458.

3. Leclerc A, Brisson BA, Dobson H. Pneumopericardium associated with a pulmonarypericardial communication in a dog. J Am Vet Med Assoc 2004;224:710-712.

\section{DIAGNOSING CONGESTIVE HEART FAILURE IN DOGS USING LIKELIHOOD RATIOS FOR PULMONARY EDEMA AND NT-PROBNP}

P.F. Lord, K. Hansson, J. Häggström. Swedish University of Agricultural Sciences, Uppsala, Sweden

Introduction/Purpose:

The likelihood ratio (LR) is a powerful measure of a diagnostic test's accuracy, and can be used on a specific patient to assess probability of disease presence/absence. It is the odds that given value of a test result would be expected in a patient with, as opposed to one without the condition. The scale of LR is logarithmic. This study compares LRs for one without the condition. The scale of LR is logarithmic. This study compares LRs for pulmonary edema by radiographic interpretation as evidence of congestive heart failure
(CHF) and published values of natriuretic peptide NT-proBNP to diagnose CHF in dogs with MR.

Methods:

Data from a previous study to evaluate reader performance for diagnosis of pulmonary edema in cavalier King Charles spaniels (CKCS) with mitral regurgitation by radiography were used to calculate dichotomous and interval LRs using a statistics program (Medcalc v.11). Dichotomous and interval LRs were measured from the receiver operating characteristic $(\mathrm{ROC})$ plot of NT-proBNP to discriminate between cardiac and noncardiac causes of respiratory signs. Using a nomogram, LRs were applied to $50 \%$ pretest probability of a hypothetical patient after clinical examination for suspected CHF to determine the posttes probabilities of $\mathrm{CHF}$ by each test and by the combined tests.

Results:

Posttest probabilities for a definitely negative radiograph (1) were $3 \%(95 \% \mathrm{Cl}, 1-5 \%)$ and for a NT-proBNP test $<908 \mathrm{pmol} / \mathrm{l}, 8 \%(95 \% \mathrm{Cl}, 0-22 \%)$. And for a definitely positive radiograph, $93 \%(95 \% \mathrm{Cl}, 88-95 \%)$, and a NT-proBNP test $>1829 \mathrm{pmol} / \mathrm{l}, 88 \%(95 \% \mathrm{Cl}$ $70-97 \%)$. A probably positive radiograph followed by a NT-proBNP $>1829 \mathrm{pmol} / \mathrm{l}$ gave a posttest probability of $93 \%$ (95\% Cl, 79-99\%).

Discussion/Conclusions:

"Definite" radiographic diagnosis was superior to NT-proBNP: LRs were further from the neutral value $(1.0)$ and $95 \% \mathrm{Cls}$ were less. LRs of indefinite radiographic interpretation $(2,3)$ were too close to 1.0 to significantly affect diagnosis. Combining the tests improved accuracy. Limitations causing unknown variabilities are different endpoints and different patients: for radiography, onset of CHF in untreated CKCS versus for NT-proBNP, treated $\mathrm{CHF}$ of different etiologies, comorbidities, and many breeds. Any dependence between the tests would worsen the combined LR.

\section{ULTRASONOGRAPHIC APPEARANCE OF THE CISTERNA CHYLI IN THE DOG}

A.-L. Etienne $^{1}$, R. Cavrenne ${ }^{1}$, A. Garcia ${ }^{2}$, V. Busoni ${ }^{1} .{ }^{1}$ Diagnostic Imaging Section, ${ }^{2}$ Internal Medicine Section, Faculty of Veterinary Medicine, University of Liège, Boulevard de Colonster, 20, Bât. B41, Sart-Tilman, 4000 Liège, Belgium

\section{Introduction/Aims:}

The cisterna chyli (CC) is a dilated and bipartite portion of the lymphatic channels coursing intimately along the abdominal aorta in the retroperitoneal space. ${ }^{1}$ The appearance of the $\mathrm{CC}$ of the dog has been described on magnetic resonance imaging. ${ }^{2}$ The aim of this poster is to describe the ultrasonographic appearance of the $\mathrm{CC}$ in two dogs with chylous ascitis and in two healthy dogs.

\section{Methods:}

Abdominal ultrasonography was realized with a $7.5-\mathrm{MHz}$ microconvex transducer. A righ or left lateral dorsal approach was used. The aorta (together with the cranial mesenteric artery in normal dogs) was used as the main landmark to localize the CC. The dogs with chylous ascites were a 4-year-old female American cocker spaniel, with immune-mediated hemolytic anemia and thrombocytopenia and a 1-year-old, spayed female whippet with recurrent pancreatitis. The two healthy dogs were a 7-year-old Beagle and a 9-year-old Jack Russell. The abdominal ultrasonography was realized within $2 \mathrm{~h}$ after the meal in the healthy dogs.

Results:

The CC was seen as a hypoechoic structure, without detectable flow, dorsal and to the right of the aorta. The CC was easily visible in the two dogs with chyloabdomen while it was more difficult to localize in the healthy dogs. The CC was tubular on the longitudinal images. more difficult to localize in the healthy dogs. The CC was tubular on the longitudinal images. On transverse images, the $C C$ is elliptic or semilunar and may partially surround the aorta.
The maximal width of the $C C$ measured on transverse images varied from $2-3 \mathrm{~mm}$ in the The maximal width of the CC measured on transverse images varied from $2-3 \mathrm{~mm}$ in the
two healthy dogs to $4-8 \mathrm{~mm}$ in the two dogs with chyloabdomen. In three patients, some efferent/afferent lymphatic vessels were seen as thin hypoechoic tubes converging on the CC. Shape and size of the CC in the same animal were seen varying during the same ultrasonography or between different exams.

Discussion/Conclusion:

The CC is visible by ultrasonography in dogs. Its visibility depends on different conditions such as disease of the lymphatic system ${ }^{3}$, quality and distance of the meal. References:

1. A.J.Bezuidenhout. Miller's anatomy of the dog. Philadelphia: WB Saunders Company, 1993.

2. Johnson VS, Seiler G. Magnetic resonance imaging appearance of the Cisterna chyli. Vet Radiol Ultrasound. 2006;47: 461-464.

3. Wachsberg RH. Ultrasonography of the retrocrural region: normal and pathological findings. Ultrasound Quarterly 2006;22: 281-290.

\begin{tabular}{|c|c|c|c|c|c|c|}
\hline \multicolumn{3}{|c|}{ Radiographic diagnosis of CHF AUC $0.92(95 \% \mathrm{Cl}, 0.89-0.93)$} & \multicolumn{4}{|c|}{ NT pro-BNP AUC $0.90(95 \% \mathrm{Cl}, 0.83-0.95) 3$} \\
\hline Interpretation & LR & $95 \% \mathrm{Cl}$ & Test & Cut-off value & LR & $95 \% \mathrm{Cl}$ \\
\hline $\begin{array}{l}\text { Dichotomized (+ CHF or } \\
-\mathrm{CHF})\end{array}$ & 5.1 (+ LR), 0.12 (-LR) & $4.2-6.2,0.08$ to -0.16 & $\begin{array}{l}\text { Dichotomized at } 1158 \\
\mathrm{pmol} / \mathrm{l}\end{array}$ & $\begin{array}{l}>1158(\mathrm{LR}+)<1158 \\
(\mathrm{LR}-)\end{array}$ & $4.6,0.18$ & $2.0-13,0.06-0.45$ \\
\hline 1 Definitely - & 0.03 & $0.01-0.07$ & Interval 1 & $<908$ & 0.09 & $0.00-0.34$ \\
\hline 2 Probably - & 0.28 & $0.19-0.41$ & Interval 2, indefinite & $908-1829$ & 1.6 & $0.5-7.0$ \\
\hline 3 Probably + & 2.1 & $1.5-2.8$ & gray zone & & & \\
\hline 4 Definitely + & 13 & $8.8-20$ & Interval 3 & $>1829$ & 7.8 & $2.1-39$ \\
\hline
\end{tabular}

AUC, area under the curve of the ROC plot; $\mathrm{Cl}$, confidence interval. 


\section{QUANTITATIVE EVALUATION OF ECHOGENICITY AND ECHOTEXTURE IN DOGS WITH LIVER FIBROSIS}

R. Sartor, M.J. Mamprim. School of Veterinary Medicine and Animal Science, São Paulo State University, Botucatu, Brazil

\section{Introduction:}

Histogram analysis can provide a quantitative assessment of a structure echogenicity and echotexture, showing the frequency distribution of echo intensity in the region of interechotexture, showing the frequency distribution of echo intensity in the region of inter-
est $(\mathrm{ROI})$ and measuring the amount of gray levels. This tool is complementary to ultrasonography decreasing its subjectivity using quantitative values. In human beings, histogram was described in the diagnosis of hepatic steatosis, showing, quantitatively, in creases in hepatic echogenicity. In cats with thyrotoxicosis, low echogenicity and high heterogeneity were described in liver parenchyma. The aim of this study was to access hepatic echogenicity and echotexture by histogram in healthy dogs and in dogs with liver fibrosis.

Methods:

Group A: Ten healthy dogs; group B: 15 dogs with chronic hepatitis or cirrhosis with slight to severe amount of fibrosis, diagnosed by histopathology (Masson's trichrome stain). To perform the histogram a computer software was used (Photoshop CS4 ${ }^{\circledR}$, Adobe); RO was fixed $\left(0.5 \times 0.5 \mathrm{~cm}^{2}\right)$. Lmean (average brightness) was used to quantify echogenicity; Nmost/Nall (all similar bright spots/total of bright spots of the ROI) and SD (range of echo variation) represented the echotexture. variation)

Results:

TABLE 1. Description of the Means $( \pm S D$ ) Variables Obtained Through Histogram Evaluation of the Hepatic Parenchyma of Healthy Dogs (Group A) and Dogs with Liver Fibrosis (Group B)

\begin{tabular}{llll}
\hline & Lmean & SD & Nmost/Nall \\
\hline Group A & $59.02 \pm 4.06 \mathrm{~b}$ & $6.87 \pm 1.40 \mathrm{~b}$ & $30.51 \pm 1.70 \mathrm{a}$ \\
Group B & $78.46 \pm 12.90 \mathrm{a}$ & $10.82 \pm 2.80 \mathrm{a}$ & $19.87 \pm 2.55 \mathrm{~b}$ \\
\hline
\end{tabular}

a, $b$-in each column, means followed by different letters differ statistically $(P<.01)$

Discussion and Conclusions:

Several diseases may lead to a diffuse hyperechoic, homogeneous or heterogeneous, liver parenchyma. Hepatic degenerations tend to increase only the echogenicity; while the presence of fibrosis tends to lead to a hyperechoic and more heterogeneous liver parenchyma. As hepatic echogenicity and homogeneity are evaluated subjectively through ultrasonography, the results may vary according to the examiner. Quantitative techniques, such as histogram, can be an important complementary tool to minimize the subjectivity of the ultrasonography. Histogram variables (Lmean, SD, Nmost/Nall) differed be tween groups, showing quantitatively the increase in the hepatic echogenicity and heterogeneity with the presence of fibrosis, while in a previous study a hyperechoic (Lmean) but homogeneous liver was observed, showing in numbers what could be subjectively uncertain.

\section{ACQUIRED PORTAL COLLATERAL CIRCULATION IN THE DOGS WITH DIFFUSE HEPATIC DISEASES}

R. Sartor, M.J. Mamprim. School of Veterinary Medicine and Animal Science, São Paulo State University, FAPESP, Botucatu, Brazil

Introduction:

Portal hypertension is the most common cause of acquired portal collateral circulation Ultrasonography has been used to describe the aspect of these vessels and to identify the origin of portal hypertension. Structural changes in liver parenchyma associated with hemodynamic disorders have been related with portal hypertension and collateral circulation in dogs. The aim of this study was to detect acquired portal collateral circulation and to identify the histopathologic aspect of the hepatic disease related.

Methods:

This study included 19 dogs, of several breeds and ages, with diffuse increase hepatic echogenicity detected by ultrasound. Portal system Doppler evaluation and percutaneous needle biopsy of hepatic parenchyma were performed. Samples were stained with hematoxylin and eosin and Masson trichrome.

toxylin and

Multiple small tortuous vessels in left renal region were described in seven dogs; among these animals one animal also had collateral vessels next to right kidneys and omenta and mesenteric regions (Fig. 1); biopsy showed four cases of cirrhosis and three of chronic hepatitis with important (moderate to severe) amount of fibrosis. Among the 12 dogs without collateral circulation, there were eight cases of chronic hepatitis and four of hepatic degeneration, but with absence or only a small amount of fibrosis.

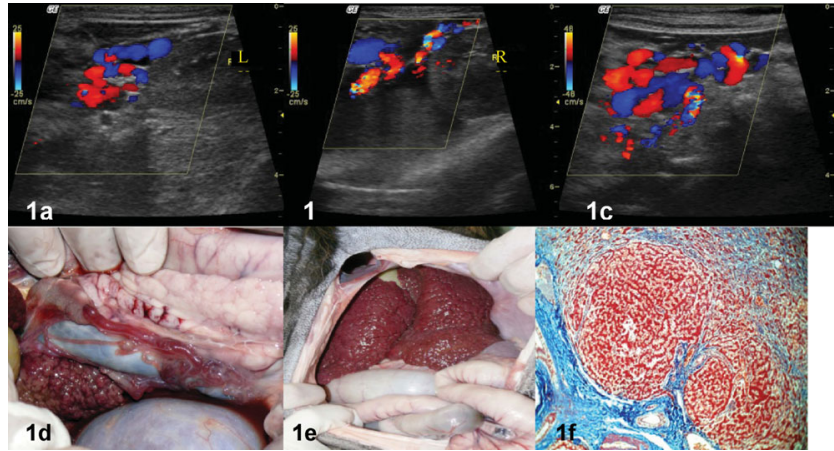

FIG. 1. Multiple acquired collateral vessels next to the left kidney-LK (1a), right kidneyRK (1b) omental and mesenteric regions (1c). Post-mortem view of multiple tortuous vessels draining to caudal vena cava (1d); macroscopic (1e) and microscopic-Masson's trichrome stain (1f) aspect of the cirrhotic liver.

\section{Discussion:}

Presence of multiple small and tortuous vessels, mainly next to the left kidney, is related to increased resistance in portal vein or caudal vena cava, leading to portal hypertension Hepatic disorders are one of the initial factors of these changes. Several diseases can lead to a similar diffuse hyperechoic liver; in these cases the presence and amount of fibrosis may not be determined only by ultrasound, histopathology with specific coloration is necessary. Whereby collateral circulation was seen only in dogs with moderate to severe fibrosis, this information may contribute to the differential diagnosis and prognosis of diffuse hyperechoic hepatic diseases, before performing the biopsy.

\section{ULTRASONOGRAPHIC DESCRIPTION OF HELICAL FLOW IN THE PORTAL VEIN OF HEALTHY CATS}

T. Gregori, P. Gómez-Ochoa, A. Corda, I. Sosa-Samper, S. Prieto-Rodríguez, S. Ruiz-Lecica Department of Animal Pathology, Hospital Clinico Veterinario de Zaragoza (HCVZ), Schoo of Veterinary Medicine, University of Zaragoza, Spain

\section{Introduction/Purpose:}

Color and spectral Doppler have been described as useful tools to evaluate main abdominal arteries and veins, including the portal vein. Normal portal vein's Doppler spectral analysis in healthy cats should include a hepatopetal (directed toward the liver) nonpulsatile flow, with a wide range of linear flow velocities across the lumen, representing a laminar blood flow. A helical portal flow pattern has been previously reported in human medicine as a variant A helical portal flow pattern has been previously reported in human medicine as a variant
of normal and associated to hepatic pathological conditions as well. The goals of this study were to identify and describe helical pattern of portal flow and to report its prevalence in healthy cats.

Methods:

In this prospective study, cats admitted through the HCVZ first-opinion clinical service between September 2006 and June 2008 were included if determined healthy based on clinical signs, physical exam and bloodwork. Patients were starved $12 \mathrm{~h}$ prior to abdominal ultrasonographic examination, which was performed without sedation. Ultrasonography was carried out with a Logic Book XP $\left(\mathrm{GE}^{\circledR}\right)$ with $4-10 \mathrm{MHz}$ microconvex transducer. Patient were positioned in right lateral recumbency, displaying porta hepatis on a longitudinal view after positioning the transducer immediately caudal to the xiphoid process. During the examination of portal flow the ultrasonographer (PGO) paid attention to avoid common Doppler artifacts and used an insonation angle on blood flow always less than $60^{\circ}$. Portal flow velocity was measured in all patients using uniform insonation method and same settings of locity was measured in all patients using uniform insonatio
PRF (range of $\pm 30 \mathrm{~cm} / \mathrm{s}$ ) and color Doppler gain $(80 \%)$ PRF (range

\section{Results:}

A total of 275 healthy cats of different breeds, aged between 2 and 9 years were examined. Helical flow within the portal vein was identified and described in 8 of 275 cats $(2.9 \%)$, these patients did not present abnormalities within the hepatic parenchyma or associated to the portal vein on bidimensional echographic mode. The helical pattern was recognizable on color Doppler examination of the portal vein due to the presence of alternating red and blue color Doppler examination of the portal vein due to the presence of alternating red and blue
bands, without the presence of aliasing. Positive and negative flows, characterized by same velocity, were noted on pulsed Doppler tracing. There was no significant difference $(P>$ $0.05)$ for mean portal flow velocity between cats with evidence of portal helical flow (12.5 \pm $4.6 \mathrm{~cm} / \mathrm{s})$ or without it $(13.8 \pm 5.2 \mathrm{~cm} / \mathrm{s})$

Discussion/Conclusions:

In human medicine literature portal helical flow has been described in normal individuals but also in those who received liver transplant, transjugular intrahepatic portosytemic shun creation or presenting with neoplastic invasion or displacement of portal vein. It has also been described as an indicator of portal stenosis when associated to increased portal flow velocities. To the authors' knowledge, no previous reports of a helical pattern associated to hepatopetal portal flow have been described in veterinary literature. Our study suggests that helical pattern associated to portal flow should be considered as variant of normal in healthy cats with a low prevalence $(2.9 \%)$, comparable to which has been reported in healthy humans $(2.2 \%)$ 


\section{CONGENITAL COLIC-CAVAL PORTOSYSTEMIC SHUNTS IN TWO CATS: IMAGING DIAGNOSIS}

M. Pivetta ${ }^{1}$, A. Holloway ${ }^{1}$, P. Neath ${ }^{2} .{ }^{1}$ Centre for Small Animal Studies, Animal Health Trust, Newmarket; ${ }^{2}$ Cave Veterinary Specialists George's Farm, West Buckland, UK

\section{Case Histories:}

Two female neutered domestic short-haired cats (2 years and 7 months of age, respectively) were referred for evaluation of suspected portosystemic shunts. Presenting clinica signs were hypersalivation and abnormal behavior in one cat and lethargy and seizures in the other. Resting bile acids were markedly elevated in both cases. Ultrasonographic findings in both cats were similar. A large-diameter, anomalous vessel extending from the cranial mesenteric vein caudally, via the middle colic vein and within the mesentery of the descending colon was present. At the level of the aortic trifurcation the vessel looped medially, parallel, and ventral to the caudal vena cava (CVC), terminating in the CVC immediately parallel, and ventral to the caudal vena cava (CVC), terminating in the CVC immediately caudal to the renal veins. Color flow Doppler demonstrated three large diameter vessels directed cranially while in the aorta flow was caudal. The morphology of the shunts was confirmed using contrast-enhanced magnetic resonance angiography in one case and intraoperative mesenteric portovenography in the other. Surgical ligation of the shunt vessel was performed successfully in both cases.

Discussion:

An extrahepatic anomalous communication represents the most common form of congenital portosystemic shunt in the cat. Although the morphology of many extrahepatic shunts is similar to that reported in the dog, it is suggested that the anatomical variation in the cat is greater. The conformation of the shunts described here is suggested to be specific to the cat although published descriptions are limited. The ultrasonographic features of a large diameter vessel, parallel to the CVC and with blood flowing in the same direction, should be distinguished from a double CVC (persistence of both supracardinal veins), a dilated splenodistinguished from a double CVC (persistence of both supracardinal veins), a dilated spleno-
renal acquired portosystemic vessel or a portocaval shunt with similar characteristics but renal acquired portosystemic vessel or a portocaval shunt with similar characteristics but
not via the middle colic vein. The termination of the shunt should be assessed to distinguish not via the middle colic vein. The termination of the shunt should be assessed to distinguish
direct communication with the CVC from more complex abnormalities, which may include direct communication with the CVC from more complex abnormalities, which may include into the left renal vein itself. It has been suggested that shunts with this morphology can be identified using one of the acoustic windows (plane 7) described as part of a standardized ultrasonographic assessment for shunts. This scanning plane is employed primarily to detect acquired spleno-renal terminations into the left gonadal vein. The limitation of this approach, with the patient in right lateral recumbency for assessment of the left sublumbar flank region, is that the shunt vessel, adjacent to the colon, is obscured by intestinal gas and may not be detected. In both cases described here, the shunt was best assessed from the right flank with the patient in left lateral recumbency. Although the shunts in these cases were identified ultrasonographically, additional angiographic studies can be used to characterize vascular malformations more completely, facilitating accurate surgical planning.

\section{ASSESSMENT OF AGING EFFECTS ON CT-GFR IN DOGS}

J.H. Chang ${ }^{1}$, H.C. Kim ${ }^{1}$, J.Y. Choi ${ }^{1}$, H.J. Choi ${ }^{1}$, H.C. Lee ${ }^{2}$, D.W. Chang ${ }^{3}$, J.H. Choi ${ }^{4}$, Y.W. Lee ${ }^{1} .{ }^{1}$ College of Veterinary Medicine, Chungnam National University, Daejeon, ${ }^{2}$ College of Veterinary Medicine, Gyeongsang National University, Jinju, ${ }^{3}$ College of Veterinary Medicine, Chungbuk National University, Cheonju-si, ${ }^{4}$ College of Veterinary Medicine, Chonnam National University, Gwangju, South Korea

\section{Introduction/Purpose:}

The purpose of this study is to assess the relationship between glomerular filtration rate (GFR) and age by using dynamic computed tomography (CT) and Patlak plot analysis in dogs.

\section{Methods and Materials:}

Fifteen client-owned dogs of nine different small breeds with age from 3 to 13 years were used based on laboratory and diagnostic imaging findings. Of the 15 dogs, there were four shih tzu dogs, three Maltese dogs, two miniature poodles, a schnauzer, a Japanese chin, a pomeranian, a Yorkshire terrier, a spitz, a mongrel dog. CT-GFR study was performed unde general anesthesia using propofol and isoflurane. A $1 \mathrm{ml} / \mathrm{kg}$ dosage of a $300-\mathrm{mgl} / \mathrm{ml}$ iohexo was administered at a rate of $3 \mathrm{ml} / \mathrm{s}$ during GFR measurement. CT-GFR was determined with a single-slice dynamic acquisition and Patlak plot analysis. The individual and global GFR values were calculated to plasma clearance per body weight $(\mathrm{ml} / \mathrm{min} / \mathrm{kg})$.

Results:

Bodyweight (mean $\pm \mathrm{SD}$ ) ranged from 2.0 to $5.7 \mathrm{~kg}(3.31 \pm 1.13 \mathrm{~kg})$. Age ranged from 3 years old to 13 years old $(7.14 \pm 3.30)$. Mean \pm SD creatinine $(0.53 \pm 0.34 \mathrm{mg} / \mathrm{dl})$, phosphorus $(4.1 \pm 1.2 \mathrm{mg} / \mathrm{dl})$, and albumin $(3.3 \pm 0.3 \mathrm{mg} / \mathrm{dl})$ concentrations and urine protein-to-creatinine ratios (all ratios were $<0.5$ ) were within reference ranges. Abdomina ultrasonography revealed small-sized renal calculi, mineralization, or renal cyst at eigh dogs. The global CT-GFR ranges shown in this study were $2.57-6.60 \mathrm{ml} / \mathrm{min} / \mathrm{kg}$. In this study, there was no trend toward weight-adjusted CT-GFR with increasing age. Conclusion:

We found no relationships between age-related kidney dysfunction in 15 dogs. Small-sized renal calculi or cysts did not affect renal function in this study. However, it is thought that a large sample size may have been required to document an age effect.

\section{RENAL RESISTIVE INDEX AND APPEARANCE OF PYELECTASIA IN 26 DOGS WITH} UPPER URINARY TRACT INFECTION

J. Ostrowska, W. Atamaniuk, W. Kinda. Department and Clinic of Veterinary Surgery, Faculty of Veterinary Medicine, Wroc"aw University of Environmental and Life Sciences, Wroc"aw,
Poland

\section{Introduction/Purpose:}

Recognizing upper urinary tract infection (UUTI) is sometimes challenging. Ultrasonographic signs of UUTI may vary, have low specificity and rarely the kidneys appearance can be normal. Inflammatory background of renal pelvis dilation, which occurs in the course of pyelonephritis, has to be differentiated from urinary tract obstruction. Previous studies on dogs $[1,2]$ revealed slight difference in RI (resistive index) value between experimentally obstructed and healthy kidney. The aim of this study was to evaluate the renal RI by means of Duplex Doppler ultrasound method in dogs with UUTI and check if pyelectasia and difference in RI values between kidneys occur in patients with pyelonephritis.

Materials and Methods:

Study group includes 26 dogs, representing different breeds, 16 males, 10 females, mean age $6.0 \pm 4.5$ years, suffering from UUTI recognized on clinical, urine, and complete blood examination. Patients with indication for ultrasound examination, but without any signs of serious or systemic diseases were assigned to control groups. Control group consisted of 44 dogs of different breeds and of both sexes (26 males, 18 females), mean age was $7.0 \pm$ 4.0 years. All animals underwent standard urinary tract ultrasound examination.

Results:

The mean $\mathrm{RI}$ value in the study group was significantly higher when compared to the contro group (for the left kidney: $0.71 \pm 0.05$ vs. $0.63 \pm 0.04$, for the right kidney: $0.7 \pm 0.07$ vs. 0.63 $\pm 0.05, P<0.001)$. In 20 dogs with UUTI $(77 \%)$, the RI value has exceeded the accepted limit of 0.71 in at least one kidney. In 15 dogs $(57 \%)$ from study group, unilateral or bilateral renal pelvis dilation was found. Healthy dogs did not reveal significant difference between $\mathrm{RI}$ value in right and left kidney $(P>0.05)$. In the study group difference in $\mathrm{RI}$ between kidneys was higher, but not statistically significant when compared to the controls $(0.037 \pm$ 0.03 vs. $0.01 \pm 0.02, P=0.06$ ). In nine dogs (34\%) with pyelonephritis, the difference in mean $\mathrm{Rl}$ between kidneys was considered as high (estimated threshold was 0.05 , calculated from the following formula: 0.01 [mean difference in RI between kidneys in healthy dogs] + $0.04[2 \times \mathrm{SD}])$. Mean RI difference between kidneys in those patients was statistically significantly higher comparing with control group $(0.09 \pm 0.04$ vs. $0.01 \pm 0.02, P<0.001)$. Six dogs $(23 \%)$ with pyelonephritis had both pyelectasia and significant difference in RI value between two kidneys (higher than 0.05).

Discussion/Conclusion:

Renal RI measurement may help to distinguish upper from lower urinary tract infection Significant differences in RI between kidneys and contemporary pyelectasia can occur in patients with pyelonephritis.

References:

1. Nyland T.G., Fisher P.E., Doverspike M., Hornof W.J., Olander H.J. Diagnosis of urinary tract obstruction in dogs using Duplex Doppler ultrasonography. Vet Radiol Ultrasound 1993:34:348-352.

2. Choi H., Effect on intravenous mannitol upon resistive index in complete unilateral obstruction in dogs. J Vet Intern Med 2003;17:158-162.

\section{UTILITY OF ANTEGRADE URETERAL STENT PLACEMENT IN UPPER URINARY TRACT OBSTRUCTION IN THREE DOGS}

G.M. Gerboni ${ }^{1}, \underline{\text { M. Brusati }}{ }^{1}$, G. Capra ${ }^{1}$, S. Scarso ${ }^{1}$, G.P. Carrafiello ${ }^{2} .{ }^{1}$ Clinica Veterinaria Malpensa, Samarate, ${ }^{2}$ Dipartimento di radiologia, Università dell'Insubria, Varese, Italy

\section{Introduction:}

Mechanical ureteral obstruction can result from an intraluminal obstruction, mural lesion or extraluminal compression. Common causes of ureteral obstruction in dogs include ureteral calculi, neoplasia, trauma, inflammation, fibrosis, congenital stenosis, acquired stricture, foreign bodies, and blood clots. The aim of this study is to evaluate the efficacy of ureteral stent placement using the antegrade technique (via percutaneous access) in three dogs. Case Histories:

The first two patients were a 13-year-old female Bouvier of Flanders referred for an unrelated problem (cutaneous mast cell tumor) and a 10-year-old male mixed breed dog referred lated problem (cutaneous mast cell tumor) and a 10-year-old male mixed breed dog referred for rectal tenesmus and anorexia. The third patient was an 11 -year-old male mixed breed
dog referred for rectal tenesmus and anorexia. The first two dogs showed mild azotaemia. dog referred for rectal tenesmus and anorexia. The first two dogs showed mild azotaemia Ultrasound examination revealed an echogenic structure at the left ureter causing subocclusion of urine outflow and involving the proximal ureteral portion in the first dog and the distal third in the second dog. Hydronephrosis and hydroureter of the left side were present. The third patient had severe azotaemia. Ultrasound showed bilateral hydronephrosis and hydroureter due to infiltrative neoplasia of the bowel. Under general anesthesia, all dogs underwent a percutaneous antegrade pyelography, allowing localization and severity of the ureteral obstruction, and a unilateral ureteral stent placement. The kit used included a sten Percuflex with double pigtail multifenestrated design, $20 \mathrm{~cm}$ in length, $6 \mathrm{~F}$ introducer, and $0.038^{\prime \prime}$ guide wire Amplatz Super Stiff (Boston Scientific Corporation, Natick, MA). Under ultrasound guidance, a renal access was obtained by percutaneous pyelocentesis, then a guide wire was passed through the needle under mobile fluoroscopic vision and advanced down the ureter into the urinary bladder. The double pigtail ureteral stent was placed in down the ureter into the urinary bladder. The double pigtail ureteral stent was placed in antegrade fashion over the guide wire and then coiled before guide wire removing. Ureteral provement of first two patients. Third patient did not obtain clinical and ultrasonographic benefits. The dog was euthanized a few days later.

Discussion:

Ureteral stent placement via antegrade pyelography was a successful technique in dogs with unilateral mural ureteral obstruction, although inclusion criteria need to be better defined with additional studies. The failure reported in the third case could be due to the different type of obstruction (extrinsic) as well as the small size of the stent used (6F) similar to data reported in human medicine. In conclusion, our preliminary results suggest that transcutaneous ureteral stent placement could be used safely and successfully to treat dogs with mural ureteral obstruction.

References:

1. Hardie EM, Kyles AE. Vet Clin Small Anim 2004; 34: 989-1010.

2. Berent AC J Vet Emerg Crit Care 2011; 21:86-103.

3. Dyer RB, Chen MY, Zagoria RJ, Regan JD, Hood CG, Kavanagh PV. Radiographics 2002; 22:1005-1022.

4. Uthappa MC, Cowan NC. Clin Radiol 2005;60:608-612. 


\section{ULTRASONOGRAPHIC EVALUATION OF TESTICULAR TUMORS IN DOGS}

P. Debiak, A. Lojszczyk-Szczepaniak. Laboratory of Radiology and Ultrasonography, Faculty of Veterinary Medicine, University of Life Sciences in Lublin, Poland

\section{Introduction:}

Among companion animals, testicular tumors are most common in dogs. The aim of this paper was to perform an ultrasonographic evaluation of testicular tumors in dogs.

\section{Materials and Methods:}

The research was conducted on 40 dogs of different breeds aged between 6 and 13 years, diagnosed in the course of an ultrasonographic examination with focal or diffuse lesions of over $5 \mathrm{~mm}$ located in the parenchyma of one or both gonads. Palpation of the testicles was also performed. The ultrasonographic examinations were carried out in the sagittal plan and the transverse plane with the use of a $10-\mathrm{MHz}$ linear transducer, and with a 7-MHz micrconvex transducer. The types of testicular lesions were categorized as a single focal lesion, a multifocal lesion, an irregular lesion surrounded by healthy parenchyma, a lesion covering the entire testicle. The size of the tumors was assessed, as well as their echotexture and echogenicity. After castration, tissue samples were collected from the gonad lesions for routine histology.

Results:

Sonographic lesions were observed in the parenchyma of 50 testicles from 40 dogs. The histopathological examination revealed: 21 cases of interstitial cell tumors (ICT), 17 seminomas (SEM), and 12 Sertoli cell tumors (SCT). During palpative evaluation, 32 dogs- $80 \%$ of the research group-were diagnosed with enlarged gonads. All diagnosed cases of SEM were accompanied by the enlargement and altered shape of the testicle. No physical lesions were observed in a total of eight subjects: six dogs diagnosed with ICT and two diagnosed with SCT, that is, $20 \%$ of the research group. Ultrasonographically, most of ICTs were the smallest tumors observed, in most cases in the form of single focal lesions. The sonographic images indicated that in 18/21 ICT cases the lesion covered not more than half of the gonad length. It was also concluded that such testicular tumors covering less than $50 \%$ of the gonad length were undetectable during the palpation of the testicles. In 11 gonads, the ICT gonad length were undetectable during the palpation of the testicles. In 11 gonads, the ICT
displayed a fine even echotexture, in $7 / 21$ testicles increased echogenicity was observed. displayed a fine even echotexture, in $7 / 21$ testicles increased echogenicity was observed. in $10 / 12$ testicles $(83.3 \%$ ) covered more than half of the gonad length and in $41.7 \%$ of the cases the imaging showed irregular lesions surrounded by healthy testicular parenchyma. Nonhomogenous echostructure was observed in 15/17 cases of SEM and 9/12 cases of SCT. Mixed echogenicity of testicular tumors was observed in 15/17 cases of SEM and 9/12 cases of SCT.

Discussion/Conclusion:

The obtained results indicated that the diagnosis of testicular neoplastic disease require the application of detailed clinical as well as ultrasonographic diagnostic techniques. Th palpative evaluation of testicular tumors is particularly difficult in the case of small lesions, whose size does not exceed $50 \%$ of the gonad length. An ultrasonographic examination allows the imaging of tumors undetectable in the course of a palpative exam. In most cases, such tumors are ICTs, usually observed in the form of focal lesions of the testicular parenchyma. The ultrasound imaging of testicular tumors is varied and is not typical for the parenchyma. The ultraso
particular type of tumors.

\section{CONGENITAL ABNORMALITES OF THE FERRET VERTEBRAL COLUMN}

P. Proks, L. Stehlík, K. Hauptman, V. Jekl. Faculty of Veterinary Medicine, University of Veterinary and Pharmaceutical Sciences Brno, Brno, Czech Republic

\section{Introduction/Purpose:}

Congenital abnormalities of the spine are frequently identified radiographically in animals. To the author's knowledge, no work has been published relating specifically to the incidence and types of congenital vertebral anomalies in ferrets. Previously described forms of congenital vertebral anomalies in other species include transitional vertebrae, hemivertebrae, block vertebrae, atlantoaxial malformations, and spina bifida. The purpose of this study was to vertebrae, atlantoaxial malformations, and spina bifida. The purpose of this study was to hospital population of ferrets. The vertebrae normally consist of seven cervical, 14 thoracic six lumbar, and three sacral vertebrae.

Methods:

The radiographic archive of the department of diagnostic imaging, University of Veterinary and Pharmaceutical Sciences Brno was searched for ferrets whose diagnostic evaluation included radiographs of the whole vertebral column. The caudal part of caudal vertabrae was not included in the study. All radiographs were reviewed by a single author (PP). Results:

A total of 69 ferrets were studied ( 40 males and 29 females). Congenital spinal abnormalities were found in 32 cases $(46.38 \%), 19$ males and 13 females. None of the ferrets with congenital vertebral abnormalities had clinical signs associated with these abnormalities. No spina bifida and block vertebra were found. No congenital abnormalities were found in cervical region. All cases (100\%) were detected in thoracolumbar and sacrococcygeal region. Hemivertebra was found in one case $(3.13 \%)$ in thoracic region (Th7). In one case $(3.13 \%), 15$ thoracic vertebrae and a normal number of cervical (C 7) lumbal (L 6) and sacra vertebrae (S 3 ) were found. In three cases ( $9.35 \%)$, transitional vertebrae with bilaterally (2) and unilaterally (1) rudimentary ribs and normal number of cervical, thoracic, lumbar, and sacral vertebrae were present. In four cases (12.5\%) were found seven lumbar vertebrae and a normal number of cervical, thoracic, and sacral vertebrae were found. In four $(12.5 \%)$ cases, unilateral (3) and bilateral (1) thoracicized first lumbar vertebra and in one (3.13\%) cases, unilateral (Th14) with rudimentary ribs were found Lumbalization of firs sacral vertebra was present in 1 case $(3.13 \%)$. In 16 cases $(50 \%)$, sacralized first cauda vertebra was found. In one case $(3.13 \%)$, a combination of a congenital abnormality with transitional first lumbar vertebra (unilateral thoracicized) sacralized last $(L 6)$ lumbar vertebra and sacralized first caudal vertebra was found.

Discussion/Conclusions:

Congenital abnormalities of the vertebral column are very common in ferret populations without associated clinical signs. Most congenital vertebral abnormalities are found in the thoracolumbar and sacrococcygeal region.

\section{${ }^{18}$ F-FDG VERSUS ${ }^{18}$ F-MISO: COMPARISON BETWEEN IMAGING UPTAKE AND} DISTRIBUTION OF BOTH DRUGS IN A BREAST CANCER MURINE MODEL

J.A. Cámara Serrano, C. Velasco Martín, E. Andrés Gómez. S. Sánchez Leal, M. Quintela F. Mulero Aniorte. Centro Nacional de Investigaciones Oncológicas (CNIO), Madrid, Spain

\section{Introduction:}

${ }^{18} \mathrm{~F}-\mathrm{FDG}$ is the most used radiotracer for cellular activity. Due to its availability, it is used in both preclinical and clinical trials. ${ }^{18} \mathrm{~F}$-MISO a hypoxic tissue marker is one of the lates drugs, from which we can expect better results than with ${ }^{18} \mathrm{~F}-\mathrm{FDG}$ in hypoxia-related studies. The aim of this study is to compare PET images of ${ }^{18} \mathrm{~F}-\mathrm{FDG}$ and ${ }^{18} \mathrm{~F}-\mathrm{MISO}$ in a breas cancer murine model.

Methods:

Eight female mice of FVB strain were used in this study. Each one of the mice has breast tumors checked with previous CT exams. ${ }^{18} \mathrm{~F}$-FDG was injected in the tail vein at a 14 $\mathrm{Mbq}$ dosage. After a capture period of $45 \mathrm{~min}$, a PET-CT whole-body study was carried out. Acquisition values were: $150 \mathrm{~mA}$ intensity, $45 \mathrm{kV}$ voltage, 8 shots in two beds, with a $360^{\circ}$ ring loop and $200 \mu \mathrm{M}$ resolution. Images were processed with 3D OSEM (PET) and Filtered-back Projection Algorithm (CT) systems and analyzed with MMWKS software, GE Healthcare Spain, Alcobendas, Madrid. The same mice were checked in an ${ }^{18} \mathrm{~F}$-MISO PET study the day after, with a similar acquisition protocol but using a $3 \mathrm{~h}$ capture period. Results:

After a visual analysis of the emission areas, a different distribution of the radiotracers was observed. The ${ }^{18} \mathrm{~F}$-FDG accumulates in the outlying zones of the tumor, while the ${ }^{18} \mathrm{~F}$-MISO flows toward deeper areas.

Discussion:

${ }^{18} \mathrm{~F}$-MISO shows a totally different distribution and uptake than the ${ }^{18} \mathrm{~F}$-FDG. ${ }^{18} \mathrm{~F}$-FDG biodistribution depends on the vascularity, like other radiotracers, and competes with glucos in metabolic cellular cycles. These cycles are avoided by the lack of oxygen; therefore, no ${ }^{18} \mathrm{~F}$-FDG is captured in hypoxic or anoxic tissues. The initial distribution of ${ }^{18} \mathrm{~F}$-MISO also depends on the vascularization, but its permanence on the tissues is related to the local oxygen tension. In this way, ${ }^{18} \mathrm{~F}$-MISO is fixed to macromolecules in hypoxic tissues with an oxygen pressure lower than $10 \mathrm{mmHg}$. This fixation depends on the nitroreductase enzyme activity. This nitroreductase is not active in anoxic tissues; therefore, the ${ }^{18} \mathrm{~F}$-MISO is unable to get fixed to the necrotic tissue. A different location of the PET signal in these studies shows a metabolically active region of the tumor, mostly in the peripheral zones. This is probably due to uncontrolled cellular divisions, shown in the capture of ${ }^{18} \mathrm{~F}-\mathrm{FDG}$. Deeper regions show more ${ }^{18} \mathrm{~F}$-MISO uptakes, probably associated to hypoxic tissues. The deepest zones of the tumors show no uptake, suggesting necrotic zones.

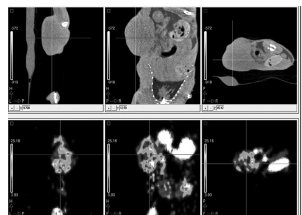

1. Distribution of ${ }^{18} \mathrm{~F}-\mathrm{MISO}$

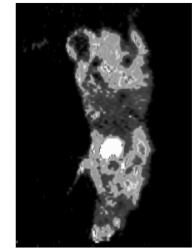

2. Different images of ${ }^{18} \mathrm{~F}-\mathrm{FDG}$ (left) and ${ }^{18} \mathrm{~F}$ MISO (right) in same animal

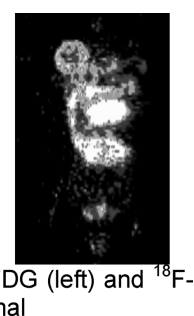




\section{RADIOGRAPHY VERSUS COMPUTED TOMOGRAPHY TO EVALUATE VERTEBRAL MALFORMATIONS IN CAPTIVE SENEGAL SOLE (SOLEA SENEGALENSIS)}

A. Barreiro ${ }^{1}$, A.M. Azevedo ${ }^{1}$, J.D. Barreiro ${ }^{1}$, M. Vila $^{1}$, A. Riaza ${ }^{2}$, S. Vázquez ${ }^{1}$, M.I. Quiroga ${ }^{1}$. ${ }^{1}$ Department of Veterinary Clinical Sciences, Veterinary Faculty, University of Santiago de Compostela, Lugo, ${ }^{2}$ Stolt Sea Farm, Lira, Carnota, A Coruña, Spain

Introduction:

The Senegal sole (Solea senegalensis) is a common marine flatfish with remarkable interes in aquaculture. Sole farming has important limitations such as a high larval mortality rate and a high incidence of skeletal malformations. In juvenile Senegal sole reared in captivity, the incidence of vertebral deformities is around $40 \%$, which is a major production constraint. The purpose of the present study is to compare digital radiography and CT images for vertebral malformations detection in different batches of juvenile Senegal sole, to test the suitability of each technique in diverse stages of development.

\section{Materials and Methods:}

For the present study three batches (A, B, and C) of Senegal sole were sampled from a fish farm in Northwest Spain. Each batch was composed of three specimens that were euthanized, measured, and frozen. The mean values of age, length, and thickness of each batch are shown in the table below.

\begin{tabular}{lllll}
\hline Batches & $\begin{array}{l}\text { Age (DAH, days } \\
\text { after hatching) }\end{array}$ & $\begin{array}{l}\text { Standard } \\
\text { length }\end{array}$ (cm) & $\begin{array}{l}\text { Total } \\
\text { length }\end{array}$ & \\
\hline A & 741 & $23.5(21.3-25.4)$ & $27.1(24.7-29.1)$ & $2(1.8-2.3)$ \\
B & 481 & $14.2(11.7-16.6)$ & $14.8(12.5-17)$ & $1.1(0.8-1.3)$ \\
C & 138 & $8.8(7.2-10)$ & $9.4(7.6-10.7)$ & $0.5(0.4-0.6)$ \\
\hline
\end{tabular}

*Standard length was measured from the tip of the snout to the caudal peduncle. **Total length was measured from the tip of the snout to the end of the caudal fin.

${ }^{\star \star \star}$ Thickness was measured in the abdominal region.

After $24 \mathrm{~h}$ of defrosting, fishes were radiographed in dorsoventral (DV) and laterolateral (LL) projections with a mammography digital system. After that a CT scan was performed. projections with a mammograp

Results and Discussion:
With respect to digital radiography, images of the three batches provided significant information about skeletal malformation in all sampled fish. LL projection greatly complemented the DV view, offering a three-dimensional (3D) perspective of the skeleton. However, the former presented interpretation difficulties because of overlapping structures, especially when skeletal abnormalities involved twisted spines. CT scan revealed a much more complete vision of skeletal conformation with discrimination of all deformed elements and vertebral alignment. It also had an exceptional 3D display of vertebrae. On the other hand, this method was not viable for small-sized fish (batch C), whose vertebral size is under the device definiwas not viable for small-sized fish (batch $\mathrm{C}$ ), whose vertebral size is under the device defini-
tion range. Radiography is an adequate method to visualize vertebral alignment and mino deformities in Senegal sole, however overlapping of some structures in animals with sever vertebral malformations render difficult interpretation. X-rays are more appropriate than $\mathrm{C}$ images for detecting anomalies in fish of batch $\mathrm{C}(138 \mathrm{DAH})$. CT scan with 3D display is the most suitable technique for visualizing severe malformations in Senegal soles of batches $A$ (741 DAH) and B (481 DAH).

\section{COMPUTED TOMOGRAPHIC DIAGNOSIS OF BRAIN ABSCESS IN A HORSE}

V.M.V. Machado, L.C. Vulcano, V.R. Babicsak, D.R. Santos, K.M. Zardo, A.S. Borges, J.J. Moreira. Universidade Estadual Paulista, Botucatu, Brazil

\section{Case History:}

A 3-month-old Quarter Horse with seizures since the age of 20 days was admitted to the Veterinary Hospital, School of Veterinary Medicine and Animal Science of UNESP, Botucatu. The animal was examined with a brain computed tomography (CT) scan in transverse sections, with 3-mm thickness and 3-mm interval, before and after intravenously administration of $2 \mathrm{ml} / \mathrm{kg}$ of an ionic-iodinated contrast medium (diatrizoate meglumine, Reliev ${ }^{\circledR}$ ). The CT images revealed the presence of a well circumscribed slightly hypoattenuating lesion (mean Hounsfield units $(\mathrm{HU})=21.3$ ), measuring approximately $4.9 \times 5.9 \mathrm{~cm}^{2}$, on dorsoventral and transverse axis (respectively). This lesion had a slightly hyperattenuating capsule (mean $\mathrm{HU}=38$ ) with moderate and homogeneous contrast intensification (mean $\mathrm{HU}=54$ ). Th abnormal brain tissue encompassed a large area, including the left parietal, temporal, and occipital brain lobes. A marked mass effect was visible with the falx cerebri deviated toward the right brain parenchyma and the left lateral ventricle displaced and distorced. In the surrounding region, a hypoattenuating amorphous and ill-defined tissue (mean $\mathrm{HU}=17$ ) was visualized. Moreover, there were irregularity and thinning of the left parietal and temporal bone. Following the CT scan, the animal was euthanized and submitted to necropsy. In the area corresponding to the CT lesion, an abscess was discovered with caseous material in which Streptococcus beta hemolytic was isolated.

\section{Discussion:}

Brain abscess is a rare disease in horses and often occurs secondarily to an infection involving other skull structures (e.g., sinusitis, otitis media, tooth roots abscesses, and traumatic injuries). In adult horses, the infectious agent most commonly isolated from brain abscesses is Streptococcus spp. The common clinical signs in animals with brain abscess are fever and neurological signs such as circling (ipsilateral), head pressing, seizures, mental changes, and focal neurological and ophthalmic tracts deficits. The diagnosis of brain abscesses is based on the clinical signs, cerebrospinal fluid analysis, and imaging modalities such as computed tomography and magnetic resonance imaging. The CT features of an intracranial abscess include a hypoattenuated area of avascular necrotic tissue. After the injection of the iodinated contrast medium, the hypoattenuated area was surrounded by a contrast enhanceiodinated contrast medium, the hypoattenuated area was surrounded by a contrast enhance-
ment region representing a tissue with increased cellularity and vascularity encapsulated by a fibrous tissue. Edema, a commonly finding secondary to brain lesions, was observed as an ill-defined hypoattenuating surrounding area. Thinning and irregularity of a diffuse portion of the parietal and temporal bone can occur by the secondary changes associated with the abscess. The CT scan facilitates the identification and evaluation of brain lesions, which is not possible through other methods of diagnostic imaging such as conventiona radiology and ultrasonography. Furthermore, CT may help to determine prognosis, as well as the appropriate treatment protocol and surgical planning in cases that surgical excision is indicated.

\section{MRI OF THE CARTILAGINOUS GROWTH STRUCTURES OF THE DISTAL RADIUS OF HORSES}

J.P. Troillet ${ }^{1}$, K. Flatz ${ }^{2}$, W. Brehm ${ }^{3}$, K. Gerlach ${ }^{3} .{ }^{1}$ Orthogen Veterinary GmbH, Düsseldorf ${ }^{2}$ Clinic for Small Animal Surgery and Reproduction, Veterinary Faculty, Ludwig-MaximiliansUniversity of Munich, ${ }^{3}$ Large Animal Clinic for Surgery, Faculty of Veterinary Medicine, University of Leipzig, Germany

\section{Introduction/Purpose:}

In horses, the epiphyseal plate of the distal radius is the most common site of angula limb deformity. The epiphyseal plate's long growth up to 30 months of age is one cause of this condition. Radiographic examination is used routinely, but cartilages structures can be imaged only indirectly. Magnetic resonance imaging (MRI) due to its extraordinary contrast of soft tissues is the best available technique for imaging intact and damaged cartilage. On account of the epyphiseal plate of the distal radius playing a decisive role in the skeletal system's degree of maturity and its common incidence of pathological changes, it seems to be reasonable to conduct an age-related examination of this region. The aim of this study was the imaging and description of the epiphyseal plate and epiphysis of the distal radius and of the apophyseal plate between radius and ulna by using MRI. The constitution and presentability of the distal region of the radius should be able to be related and characterized by possible age-related structural changes.

by possible age-related st

Twenty-eight cadaver limb segments including the distal radius of 28 orthopedically sound horses of diverse breeds aged between 2 days and 17 years were examined by MRI. Th limbs were examined in four different sequences (T1w-SE, T2w-TSE, PD-SE, T2 DESSGE) and in two planes (sagittal, dorsal). Three of these physis were additionally examined histologically and compared with MRI. The region of growth with respect to the physis of the distal radial bone was documented descriptively. Age-related changes regarding the cartilaginous structures within this region were distinguished. The average thickness of the obtainable cartilage zone of the physis was measured consecutively using two sequences (T1w-SE, T2 DESS-GE). The results were compared with each other, the increasing age of the examined samples also being considered.

Results:

The hyaline cartilage of the examined area could be clearly defined by the sequences used. Cartilaginous structures of the epiphyses and apophysis were considered to be well assessed with MRI. In young foals, proximal to the physeal cartilage a zone of provisional calcification could be documented, whereas a hypointense epiphyseal subchondral bone plate was visible distally adjacent to this. In adult horses, physeal scars in that region of the growth plate were clearly detectable. The sample materials could be classified into five groups due to age-related varieties. With the help of a correlation analysis, a strong connection between the age of the evaluated individuals and the decreasing thickness of the cartilaginous physis could be demonstrated.

Conclusion:

MRI is an applicable method to assess the cartilaginous structures of the growth region of the distal radial bone of horses. 


\section{EFFECT OF DIFFERENT HEAD AND NECK POSITIONS ON RADIOLOGICAL CERVICAL INTERVERTEBRAL FORAMINA DIMENSIONS IN STANDING HORSES}

D. Berner, W. Brehm, K. Gerlach. Large Animal Clinic for Surgery, Faculty of Veterinary Medicine, University of Leipzig, Germany

\section{Introduction/Purpose:}

Pathological reduction of intervertebral foramina size can be caused by ventral enlargement of the cervical facet joints. This aspect is used in plain radiographic evaluation to identify pathologically changed articular process joints. In humans, it is known that movement of the neck induces variation of the intervertebral foramina dimension. Specifically, extension leads to a decrease and flexion to an increase in intervertebral foramina size. A recen computer tomographic study confirmed similar results in cadaver cervical spines of adult warmblood horses. The aim of this study was to determine the changes of intervertebra foramina dimensions in three different head and neck positions in living horses.

Materials and Methods:

Twenty-five horses without any clinical signs of neck diseases were examined. Lateral computed radiographs were taken from the mid- and caudal portion of the neck in the three following positions: the mouth of the horses at the level of the shoulder joint (physiologica $\mathrm{S} 1$ ), the carpal joint (flexion S2), and the withers (extension S3). Compared to the horizontal line the following angles resulted: for $\mathrm{S} 1-45^{\circ}, \mathrm{S} 2-0^{\circ}$, and $\mathrm{S} 3-60^{\circ}$. From these radiographs the length of the vertebral body (D1), the height of the cranial intervertebral foramen (D2) and the height of the caudal intervertebral foramen (D3) were measured. Also, the angles between the lengths of the vertebral bodies (D1) of adjacent vertebrae were calculated. All measurements were taken three times.

Results:

Lengths of the vertebral bodies as well as heights of the cranial intervertebral foramina showed no variations between the three different head and neck positions. Compared to extension and to the physiological position, flexion caused an increase in height of the cauda intervertebral foramina of the third $(P<0.05)$ to seventh $(P<0.001)$ cervical vertebrae. Only the height of the caudal intervertebral foramina of the seventh cervical vertebrae $(P<0.05)$ in the physiological position was wider than in extension. The greatest change in angles was found between the sixth and seventh cervical vertebrae.

Discussion/Conclusions:

Flexion increases the height of the caudal intervertebral foramina of the cervical vertebrae in horses. Our study demonstrates the importance of the head and neck position in radiographic examination of equine cervical spines. To prevent manipulation, standardizing the head and neck position for radiographic examination of the neck should be considered.

\section{TRANSABDOMINAL ULTRASOUND FINDINGS IN HORSES WITH CHRONIC OR RECURRENT COLIC AND/OR WEIGHTLOSS-A RETROSPECTIVE STUDY}

I. Tosi ${ }^{1}$, G. Bolen ${ }^{2}$, L. Evrard ${ }^{2}$, A.-L. Etienne A.-L. ${ }^{2}$, V. Busoni ${ }^{2} .{ }^{1}$ Equine Clinic, ${ }^{2}$ Diagnostic Imaging Section, Faculty of Veterinary Medicine, University of Liège, Sart-Tilman, Liège Belgium

\section{Introduction/Purpose:}

The aim of this poster is to describe transabdominal ultrasound (US) findings and their prevalence in horses admitted for chronic or recurrent colic or for weight loss.

Methods:

Medical records of horses having undergone a complete transabdominal US in a nonemergency setting were retrospectively reviewed over a 4-year period. Horses were included if they were admitted with a history of chronic or recurrent colic or weight loss. Horses with diarrhea without weight loss were not included. The abdominal US had been realized with a $3.5-\mathrm{MHz}$ convex transducer after clipping. Higher frequency transducers were also used when possible to assess the gastrointestinal wall. The caudo-ventral thorax was also assessed in all horses for presence of free fluid. A complete thoracic US was realized if free fluid was visible or if clinical symptoms suggested a concurrent respiratory

disease.

Sixty-eight horses responded to selection criteria (mean age 11.5 years). Twenty-four horses had a history of weight loss, 51 of chronic or recurrent colic. In two horses no abnormality was seen, in two an extra-abdominal lesion was the only US abnormality detected. Thickening of the gastrointestinal wall was seen in $53 \%$ cases, mainly affecting large intestine (23 horses). Peritoneal fluid was judged mildly to severely increase in volume in 27 horses. Twenty-two horses had intestinal motility subjectively judged abnormal. Thoracic US showed abnormalities in $21 \%$ horses.

\section{Discussion/Conclusions:}

The high prevalence of abnormal US findings in this population demonstrates the usefulness of a complete abdominal US in horses with suspected nonacute abdominal disease. Thoracic US often added supplementary information indicating the value of a concurrent US assessment of at least a ventro-caudal thoracic window.

\section{COMPARISON OF VARYING INJECTION RATES OF SALINE CHASERS ON INTRAVASCULAR CONTRAST ENHANCEMENT FOR DYNAMIC CT IN CATTLE}

K. Lee, K. Yamada. Department of Clinical Veterinary Science, Obihiro University of Agriculture and Veterinary Medicine, Japan

Introduction/Purpose:

The use of a saline chaser results in greater efficiency of the contrast medium by avoiding accumulation of the contrast medium at the injection site. Such increased efficiency can be used to obtain enhancement or to reduce the volume of the contrast medium used. A reduction of the volume of the contrast medium can be economically beneficial, particularly in cattle because they require a large volume of contrast medium. However, there was no study about effect of different saline chaser techniques on the pattern of contrast enhancement in cattle. The purpose of this study was to investigate the effect of saline chasers on the intravascular contrast enhancement when administered at three different rates.

Methods:

Dynamic computed tomography (CT) was performed in five normal Holstein calves. In a crossover method, group A was administered only contrast medium $(600 \mathrm{mgl} / \mathrm{kg}, 4 \mathrm{ml} / \mathrm{s}$ ). Groups B, C, and D were administered contrast medium at $30 \%$ reduction followed by saline chasers injected at 2,4 , and $8 \mathrm{ml} / \mathrm{s}$, respectively. Attenuation values were obtained from the right and left maxillary arteries and dorsal sagittal sinus.

Results:

Maximum enhancement value and mean value of the enhancement plateau obtained from maxillary arteries were significantly lower in group $B$ than in other groups. The duration of the enhancement plateau was longer in group $\mathrm{C}$ than in groups $\mathrm{B}$ and $\mathrm{D}(P<0.05)$ Discussion/Conclusion:

Saline chaser at the rate of $4 \mathrm{ml} / \mathrm{s}$ is advocated when considering maximum contrast en hancement and prolonged duration of the enhancement plateau in dynamic CT of cattle.

\section{CONTRAST-ENHANCED ULTRASOUND ANALYSIS OF RENAL PERFUSION IN NORMAL MICROPIGS}

K. Yi, S. Ji, J. Kim, J. Yoon, M. Choi. Department of Veterinary Diagnostic Imaging, College of Veterinary Medicine and Research Institute for Veterinary Science, Seoul National University, Seoul, South Korea.

\section{Introduction:}

Contrast-enhanced ultrasound is one of the proper methods to analyze renal perfusion. The aim of this project was to evaluate the renal perfusion pattern and perfusion dynamics in the normal micropigs with ultrasonographic contrast media.

Methods:

Eight clinically healthy micropigs (11-13 kg body weight, 3-4 month of age) were studied. All micropigs underwent a physical examination, and basic blood analysis was performed. The micropigs were preanesthetized with a compound of tiletamine, zolazepam, tramadol, and medetomidine and maintained on intravenous propofol. The kidney was evaluated with B-mode harmonic ultrasound. All micropigs received an intravenous bolus of a microbubble contrast medium $\left(0.06 \mathrm{ml} / \mathrm{kg} \mathrm{BW}\right.$, SonoVue $\left.{ }^{\circledR}\right)$. The contrast medium was administrated via a three-way stopcock and this was followed by a rapid bolus of $5-\mathrm{ml}$ saline. With the beginning of injection, images were captured for approximately $5 \mathrm{~min}$ at a rate of one frame per $5 \mathrm{~s}$ for the first $120 \mathrm{~s}$ and then one frame per $30 \mathrm{~s}$.

Results:

A significant difference in perfusion was detected between renal cortex and medulla. The renal parenchyma was enhanced in two phases. First, cortex was enhanced, followed by a more gradual enhancement of the medulla. Mean time to peak intensity for the renal cortex from injection was $16.3 \mathrm{~s}$, within renal medulla mean time to peak was $26.3 \mathrm{~s}$.

Conclusion:

Contrast-enhanced harmonic ultrasound is a noninvasive imaging technique to analyze the differentiation between cortical and medullary renal perfusion patterns. The findings of these data can be used as normal reference values and may prove useful in the evaluation of micropigs with renal disease. 


\section{HEPATIC ABSCESS ASSOCIATED WITH OMPHALOPHLEBITIS IN A LAMB-CASE REPORT}

R.V. Santos, V.M.V. Machado, R.M. Cavalcanti, K.M. Zardo, R.M. Amorin. Faculdade de Medicina Veterinária e Zootecnia - UNESP, Botucatu, Brazil

\section{Case History:}

A 15-day-old male mixed breed lamb was admitted to the Veterinary Hospital, School of Veterinary Medicine and Animal Science of UNESP Botucatu The animal presented with hyperthermia, a 2-cm purulent wound of the umbilical remnant, a palpable enlarged intraabhyperthermia, a 2-cm purulent wound of the umbilical remnant, a palpable enlarged intraab-
dominal structure, paraparesis, and decreased reflexes in forelimbs. The cranial nerves and spinal reflexes in hindlimbs were preserved. There were no abnormalities on radiog raphy exam of the thoracic, lumbar, and sacral spine. Therefore, an ultrasound exam was performed and revealed a thickened tubular structure with coarse echotexture at 1.4- $\mathrm{cm}$ depth, extending from tissues surrounding the subcutaneous umbilicus into the right hepatic lobe. It was determined to be enlarged filled umbilical vein (Fig. 1). In the termination of this vein, a mixed echogenicity region with internal hyperechoic gas echoes was observed indicating an anaerobic infection suggestive of an ascending omphalophlebitis with hepatic abscessation. The size and shape of liver was judged to be normal, but there was anothe hyperechoic focal $1.1-\mathrm{cm}$ structure within the left hepatic lobe without internal vascularization when interrogated by color Doppler exam, suggesting a second hepatic abscess (Fig. 2). An additional round $1.5-\mathrm{cm}$ anechoic structure with hyperechoic walls was detected displaying distal acoustic enhancement within the right hepatic lobe indicating a capsular cystic (Fig 3). The patient was discharged with treatment of florfenicol and prescribed physical therapy 3). The patient was discharged with treatment of florfenicol and prescribed physical therapy
on lumbar spine and forelimbs. Follow-up inquiry reported the lamb to be healthy after 2 months.

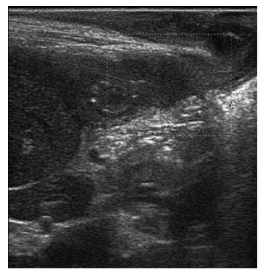

Figure 1. Umbilical vein

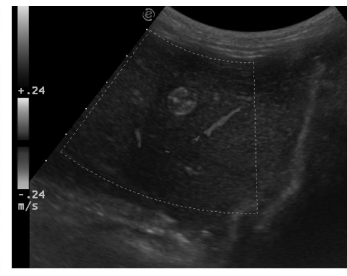

Figure 2. Abscess

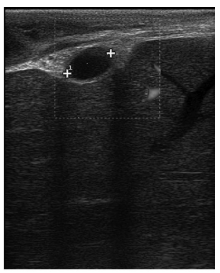

Figure 3. Capsular cystic
Discussion:

Routine sonographic examination of the umbilical region in neonates consists of complete examination of umbilical remnant, umbilical vein, umbilical arteries, and urachus. The um bilical remnant infection cases may have a favorable prognosis if there is no associated infection of umbilical vein and liver. The majority of umbilical vein infections do not extend cranially into the liver, but ultrasound examinations should be performed carefully throughout the liver for thorough examination. In this case, the sonographic evaluation was useful in discovering the umbilical vein infection and hepatic abscess due ascending omphalophlebitis and targeting appropriate medical therapy.

\section{COMPUTED TOMOGRAPHIC AND RADIOLOGIC ASPECTS OF MANDIBULAR OSTEOMYELITIS IN MARSH DEER (BLASTOCERUS DICHOTOMUS)}

Z. Bortolini, V.M.V. Machado, R.V. Santos, C.R. Teixeira, J.L. Linardi, L.C. Vulcano. School of Veterinary Medicine and Animal Science - UNESP, Botucatu, Brazil

\section{Case History:}

An 8-year-old male marsh deer was admitted to the Wild Animal Medical and Research Center with a history of weight loss and wasting, with a swelling of the left mandibula bone, missing third premolar and first molar teeth and gum erosions. Additionally, the buccal mucosa was accumulating food material and had focal purulent discharge. On radiography, there was proliferative bone reaction and bone lysis of the left mandible associated with an increased volume of soft tissue with internal air collections. Computed tomography (CT) exam revealed expansive bone lysis in left mandible and soft-tissue swelling with presence of air. Cytological and microbiological material was collected for definitive diagnosis. The cytologic diagnosis was confirmed as Actinomyces pyogenes and the animal was euthanized considering the poor prognosis and extent of infection.

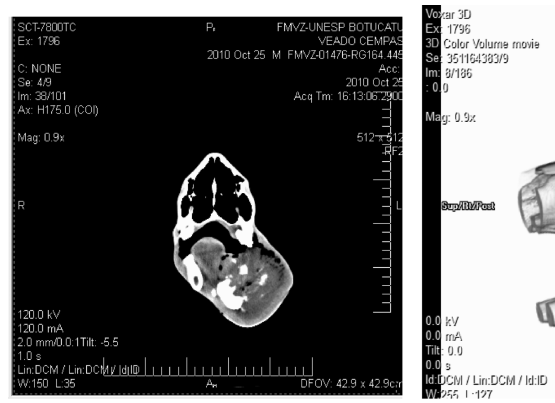

Figure 1

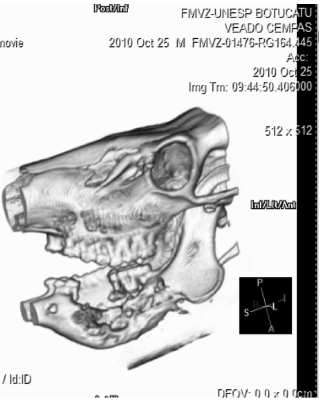

Figure 2
Fig. 1. Head axial section of first molar area showing expansive bone lysis, tooth loss and soft-tissue swelling with presence of air. L, left.

Fig. 2. Three-dimensional CT volume reconstruction illustrating extensive bone reaction in left mandible.

\section{Discussion:}

A. pyogenes is a bacterium commonly associated with osseous proliferation and destruction often with purulent production, particularly in domestic ruminants, but has not previously been reported in the Marsh Deer species. It is a common opportunistic bacterium of oral mucosa of the domestic animals, causing severe infections and spreading by blood to other tissues. In this case, the use of CT, and particularly the CT three-dimensional reconstruction, tissues. In this case, the use of $\mathrm{CT}$, and particularly the $\mathrm{CT}$ three-dimensional reconstruction,
provided more detailed information on the degree of bone destructive and extent of the provided
lesion. 
RADIOGRAPHIC AND TOMOGRAPHIC DIAGNOSIS OF PNEUMOTHORAX IN ANTEATER (TAMANDUA TETRADACTYLA)

N.F. Sesoko, V.M.V. Machado, K.M. Zardo, H.S. Oliveira, G.D.P. Soares, C.R. Teixeira. School of Veterinary Medicine and Animal Science- UNESP, Botucatu, Brazil

Case History:

An adult female anteater was taken to the Veterinary Hospital after vehicular trauma and it presented with head injury, epistaxis, and prostation. The animal was restrained physically presented with head injury, epistaxis, and prostation. The animal was restrained physically and chemically to perform imaging. Latero-lateral and ventral-dorsal thoracic radiography demonstrated the cardiac silhouette shifted to right hemithorax, with dorsal deviation of heart in relation to sternum. There was volume decrease of the left lung lobes with increase of lung tissue radiopacity and retraction of pleural margins in relation to the dorsa wall of thoracic cavity. There was increased radiolucency in the pleural space and cauda displacement of diaphragm to the left hemithorax. Computed tomography was performed with helical transverse sections of 5-mm thick with 5-mm increments. Tomographic images
revealed mediastinal, trachea, and heart deviation to the right side by air in pleural space revealed mediastinal, trachea, and heart deviation to the right side by air in pleural space
of left hemithorax, causing decrease in lung volume and consequent increase in density of left lung lobes (Figs. 1and 2). There was also complete fracture with bone axis deviation in 10th-12th left ribs as illustrated in three-dimensional reconstruction (VOXAR 3D ${ }^{\circledR}$ version 6.3; Fig. ??). With the diagnosis of pneumothorax established, pneumocentesis was performed. Repeat thoracic radiography examination revealed increased contact of the heart silhouette with sternum, adequate expansion of the lung lobes, and appropriate position of diaphragmatic crus. After observation period of 5 days, the anteater was reintroduced in its natural environment.

Discussion:

The anteater suffered traumatic rib fractures and associated pneumothorax, but the anima presented with no clinical sign characteristic as dyspnea, restlessness, or cyanosis as occurs in most domestic animals with this degree of lung compromise. As only the left lung lobes were affected, it is assumed that the hemithorax of anteaters are totally divided by pleura, and because of this, the anteater maintained adequate pulmonary function and was not exhibiting respiratory symptoms. By eliminating overlapping tissue inherent in conventional radiography, computed tomography was more sensitive to confirm the diagnosis and evaluate the extent of injury.

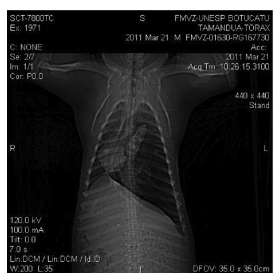

Figure 1

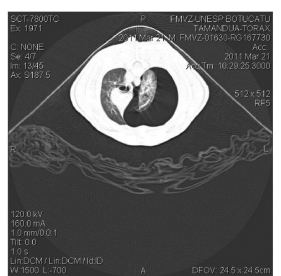

Figure 2

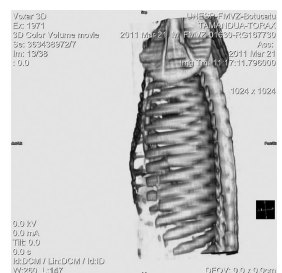

Figure 3
DIAGNOSTIC VALUE OF COMPUTED TOMOGRAPHY AS A NONINVASIVE IMAGING TECHNIQUE AS DEMONSTRATED IN KNUT, THE POLAR BEAR

G. Galateanu $^{1}$, C.A. Szentiks ${ }^{1}$, F. Göritz ${ }^{1}$, G. Fritsch ${ }^{1}$, Andre Schüle ${ }^{2}$, T. B. Hildebrandt ${ }^{1}$. $^{1}$ ${ }^{1}$ Leibniz Institute for Zoo and Wildlife Research; ${ }^{2}$ Zoological Garden, Berlin, Germany

\section{Introduction/Purpose:}

Knut, the world's most famous polar bear, died unexpectedly, at the age of 4 , on March 19,2011 , following a fit of apparent seizures. Polar bear, the largest bear and the only marine species in the otherwise terrestrial Ursidae family, is not a customary candidate for a computed tomographic (CT) examination.

Methods:

The cause of death was investigated by CT examination, as a noninvasive method, prior to necropsy. A state-of-the-art, 128-slice CT scanner was used to perform the challenging whole body examination on the $305-\mathrm{kg}$ Knut.

Results:

The high-resolution CT images revealed cerebral lateral ventricular asymmetry in otherwise structurally normal-looking brain. Necropsy and histological examination of the formalin fixed brain revealed panmeningoencephalomyelitis, nevertheless it showed no ventricular asymmetry and dilatation.

Discussion/Conclusions:

The importance and technical advantages of the CT as a noninvasive tool were demonstrated in this study. Once the brain was removed at necropsy, the cerebral fluid pressure was released and the ventricles collapsed. The neurologic symptoms and the findings in the prior CT examination were instrumental in directing the pathologists to search for possible related brain disorders, and to evaluate if there is any association of this lateral ventricle asymmetry with other structural pathologies, which caused Knut's apparent seizure that eventually led to his death.

Fig. 1: Scout image of an adult female anteater.

Fig. 2: Axial section of $4^{\circ}$ intercostal space revealing air in left pleural space.

Fig. 3: Thoracic 3D reconstruction showing fracture in 10th-12th left ribs 